
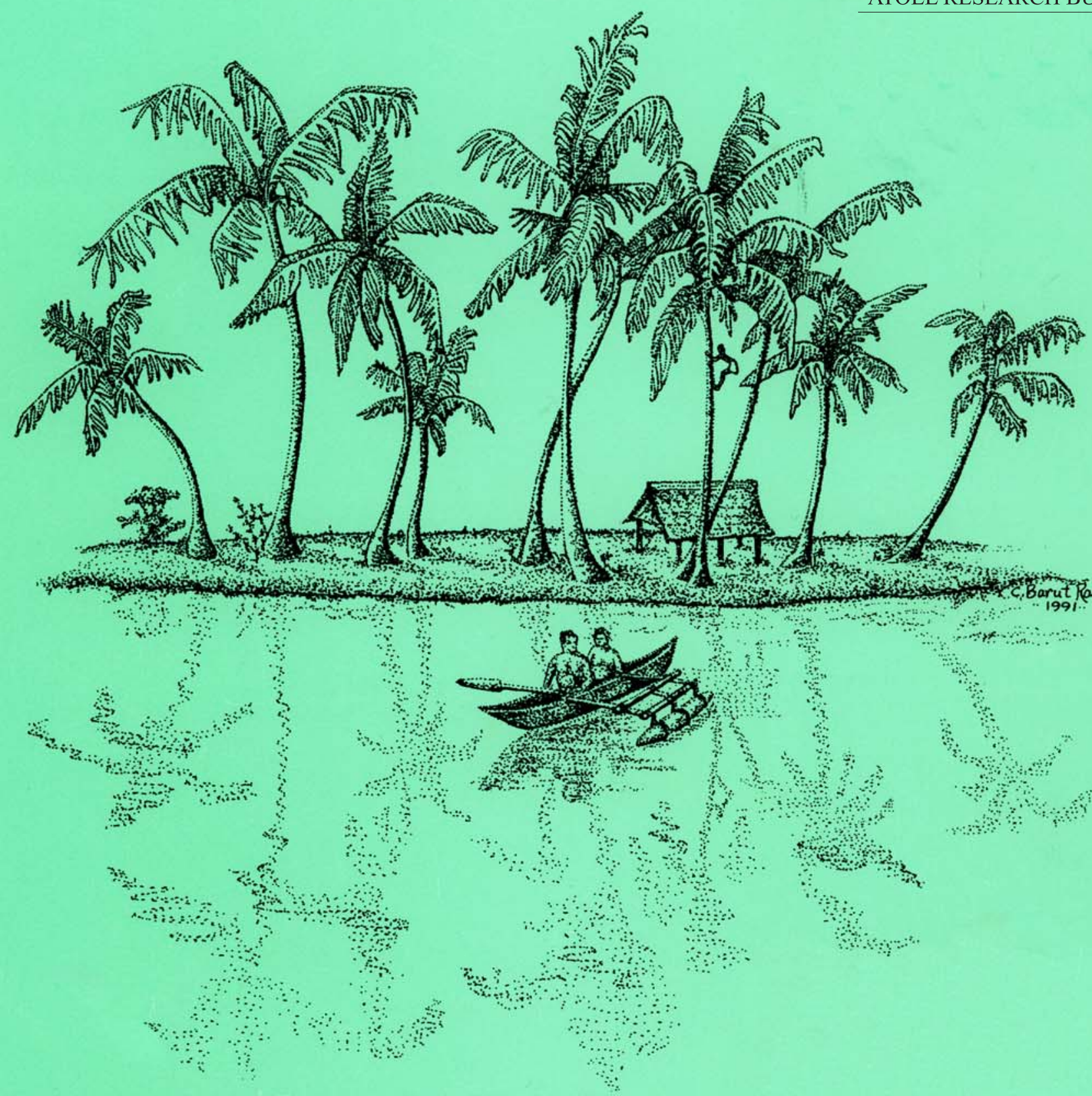

AN UPDATED ACCOUNT OF THE VASCULAR FLORA OF

THE ILES EPARSES (SOUTHWEST INDIAN OCEAN)

Vincent Boullet, Jean Hivert,

and Luc D. B. Gigord

\section{ATOLL \\ RESEARCH}

Smithsonian Institution
Scholarly Press

BULLETIN 


\title{
AN UPDATED ACCOUNT OF THE VASCULAR FLORA OF THE ILES EPARSES (SOUTHWEST INDIAN OCEAN)
}

\author{
Vincent Boullet, Jean Hivert, \\ and Luc D. B. Gigord
}

Atoll Research Bulletin No. 614 • 31 January 2018

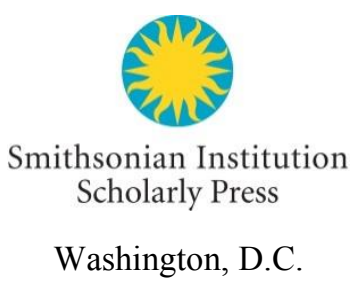


All statements made in papers published in the Atoll Research Bulletin are the sole responsibility of the authors and do not necessarily represent the views of the Smithsonian Institution or of the editors of the bulletin. Articles submitted for publication in the Atoll Research Bulletin should be original papers and must be made available by authors for open access publication. Manuscripts should be consistent with the "Author Formatting Guidelines for Publication in the Atoll Research Bulletin." All submissions to the bulletin are peer reviewed and, after revision, are evaluated prior to acceptance and publication through the publisher's open access portal, Open SI (http://opensi.si.edu).

\section{Published by SMITHSONIAN INSTITUTION SCHOLARLY PRESS}

P.O. Box 37012, MRC 957

Washington, D.C. $20013-7012$

https://scholarlypress.si.edu/

The rights to all text and images in this publication are owned either by the contributing authors or by third parties. Fair use of materials is permitted for personal, educational, or noncommercial purposes. Users must cite author and source of content, must not alter or modify the content, and must comply with all other terms or restrictions that may be applicable. Users are responsible for securing permission from a rights holder for any other use.

ISSN: 0077-5630 (online) 


\title{
AN UPDATED ACCOUNT OF THE VASCULAR FLORA OF THE ILES EPARSES (SOUTHWEST INDIAN OCEAN)
}

\author{
VINCENT BOULLET ${ }^{1}$, JEAN HIVERT ${ }^{2}$ and LUC D. B. GIGORD ${ }^{2}$
}

\begin{abstract}
The terrestrial vascular flora of the Iles Eparses (southwest Indian Ocean) was widely underestimated until the end of the $20^{\text {th }}$ century. Thanks to recent field surveys and plant inventories conducted between 2004 and 2017 by the Conservatoire Botanique National de Mascarin (CBNM), a total of 250 taxa (120 native, 107 alien and 23 cryptogenic) belonging to 66 families and 178 genera, was recorded. This represents an increase of $42-313 \%$ for the four surveyed islands. While Bassas da India does not show any terrestrial flora, the flora of Europa includes 94 taxa (47 native, 39 alien and 8 cryptogenic), that of Juan de Nova 147 taxa (62 native, 69 alien and 16 cryptogenic), that of the Iles Glorieuses 137 taxa (67 native, 61 alien and 9 cryptogenic) and that of Tromelin only 21 taxa ( 7 native and 14 alien). The native plant species density is lower for Europa $\left(1.56 / \mathrm{km}^{2}\right)$ and Tromelin $\left(7 / \mathrm{km}^{2}\right)$ and highest for Juan de Nova and the Iles Glorieuses $\left(12.4 / \mathrm{km}^{2}\right.$ and $15.3 / \mathrm{km}^{2}$ respectively). The native vascular floras of Europa, Juan de Nova and the Iles Glorieuses are mainly characterized by pantropical (24-30\%) and Indo-Pacific taxa (13-22\%). In Juan de Nova and Europa, the Malagasy elements sensu stricto are strongly represented (13-15\%), while taxa in the Iles Glorieuses are confined to the southwest of the Indian Ocean (30\%). Europa, Juan de Nova and Tromelin seem to host local endemics species while one taxon found both on Juan de Nova and Grande Glorieuse is endemic to these two islands. The alien flora of Europa, Juan de Nova and the Iles Glorieuses comprise between $64 \%$ and $97 \%$ naturalized taxa while that of Tromelin hosts $50 \%$ of non-naturalized alien taxa. The higher percentages of cultivated alien taxa are found in Juan de Nova and Tromelin (56\% and $71 \%$, respectively).
\end{abstract}

\section{INTRODUCTION}

\section{General Context}

The Iles Eparses comprise five island territories located in the southwest Indian Ocean, close to Madagascar. Four are located in the Mozambique channel - from South to North: Europa, Bassas da India, Juan de Nova and the Iles Glorieuses - while the fifth, Tromelin, is located in the North of the Mascarene archipelago (Caceres, 2003) (Figure 1).

Each one culminates at a low altitude: about 12 meters for Europa, about $10 \mathrm{~m}$ for Juan de Nova, about $14 \mathrm{~m}$ for Iles Glorieuses and about $8 \mathrm{~m}$ for Tromelin. Located between $22^{\circ}$ south for Europa and $11^{\circ}$ south for the Iles Glorieuses (Figure 1), the Iles Eparses fall into a climatic gradient spanning from a sub-arid climate for the most southern island to a more humid climate for the most northern island (Figures 2-5).

\footnotetext{
${ }^{1}$ Vourlhat 43230 Frugières-le-Pin, France, and EA 7462 Géoarchitecture, Université de Bretagne Occidentale, 29200 Brest, France.

${ }^{2}$ Conservatoire Botanique National de Mascarin, 2 rue du Père Georges, Les Colimaçons, 97436 Saint-Leu, île de La Réunion, France.
} 


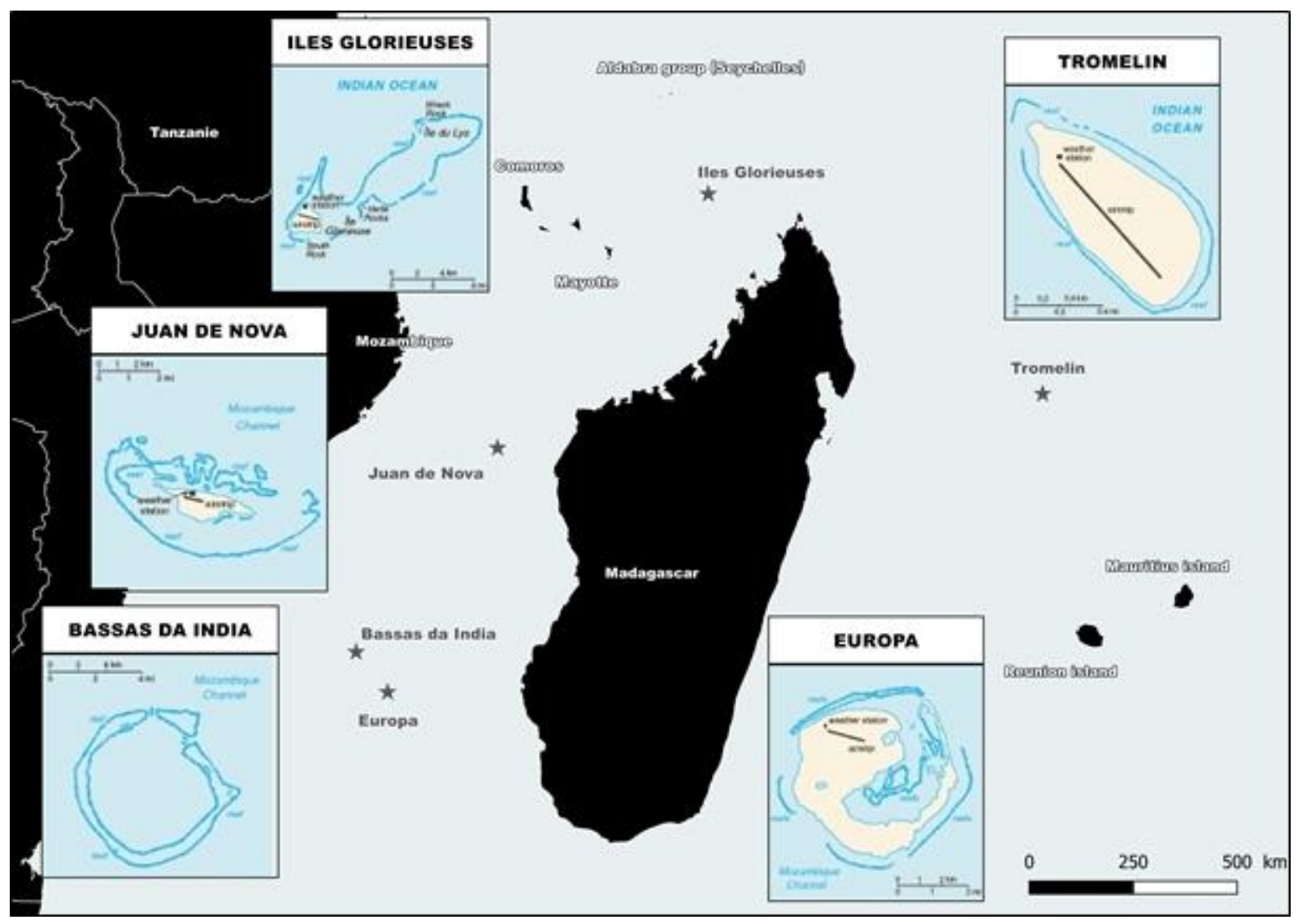

Figure 1. Location of the Ines Eparses in the southwest Indian Ocean.

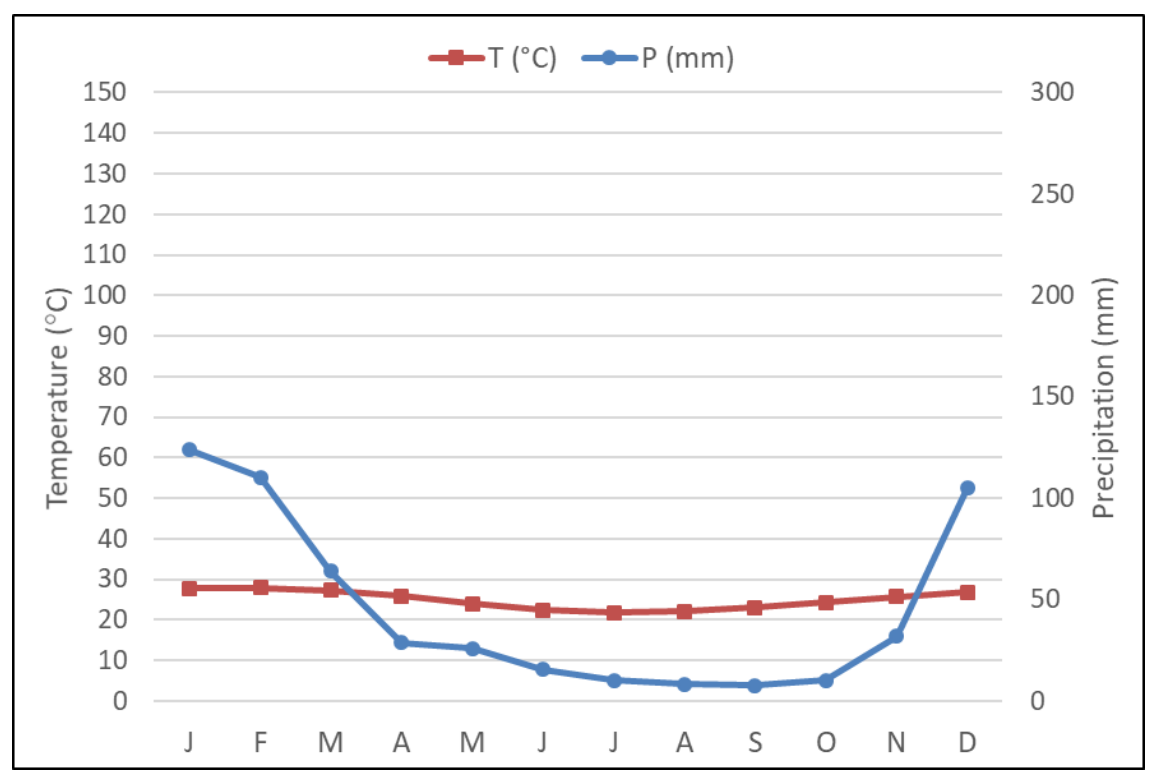

Figure 2. Gaussen ombrothermic diagram for Europa (average of 49 years [1951 - 1999]).

2 


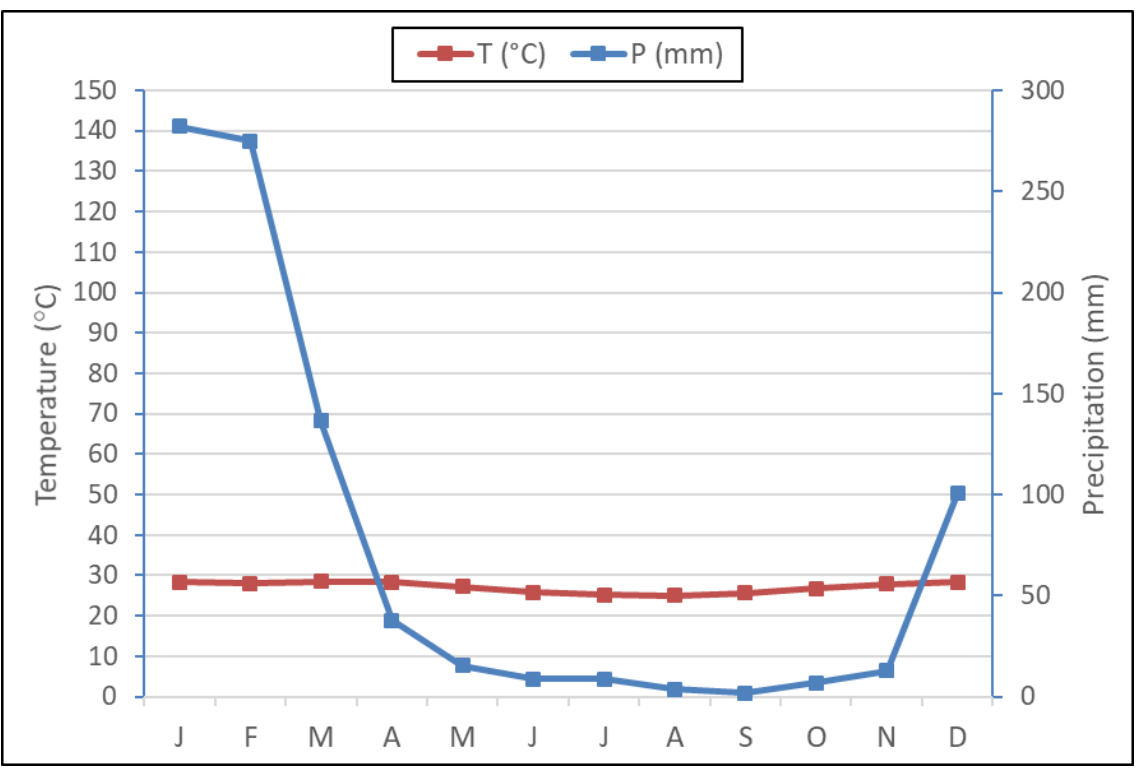

Figure 3. Gaussen ombrothermic diagram for Juan de Nova (average of 26 years [1974 - 1999]).

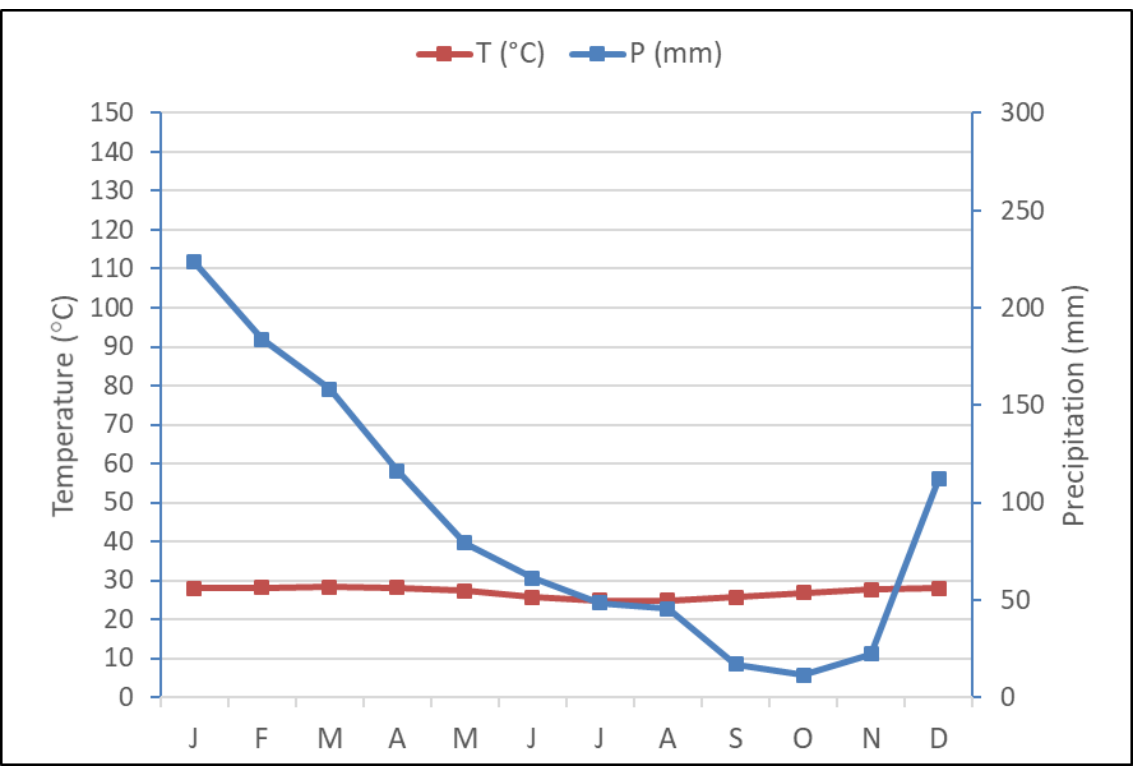

Figure 4. Gaussen ombrothermic diagram for Iles Glorieuses (average of 49 years [1951 - 1999]). 


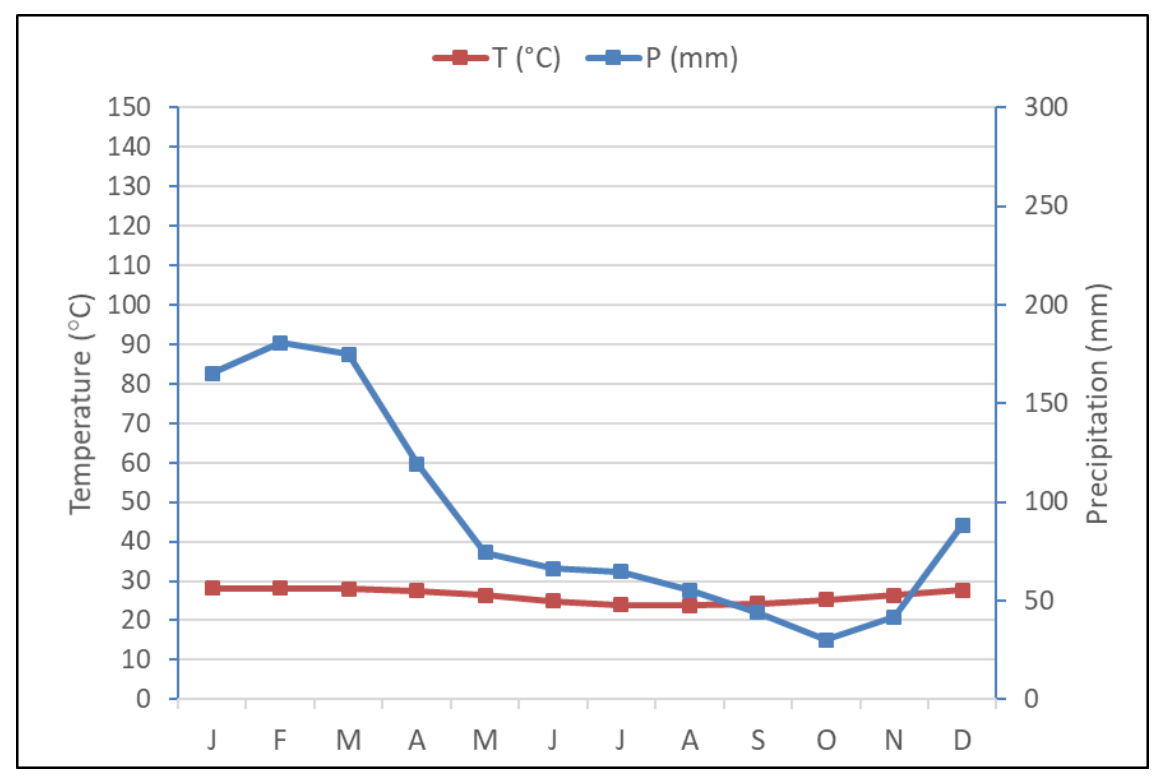

Figure 5. Gaussen ombrothermic diagram for Tromelin (average of 58 years [1955 - 2012]).

They are subjected to a regime of tropical influence, with two prevailing seasons: a dry and relatively fresh season (from May to November) and a warm and humid season (from December to April). All are located in the zone of formation and circulation of tropical depressions and cyclones (Delépine et al., 1976; Caceres, 2003). Europa, Bassas da India and the Iles Glorieuses each consist of a coral reef built on an ancient volcanic cone. They are remnants of old intra-plate volcanoes, nowadays morphologically present in the form of atolls (Goldberg, 2016) which is not the case for Juan de Nova and Tromelin. All these islands are relatively young, about 125,000 years old (Caceres, 2003). They administratively belong to the $5^{\text {th }}$ district of the French Southern and Antarctic Land (Terres Australes et Antarctiques Françaises or TAAF), a French Oversea Territory, since 2007 (TAAF, 2011, 2016). In spite of their reduced terrestrial area (from $30 \mathrm{~km}^{2}$ for Europa to $1 \mathrm{~km}^{2}$ for Tromelin; absence of terrestrial area for Bassas da India), these islands share an exceptional natural heritage including many marine and terrestrial endemic species. At a regional scale the Iles Eparses are some of the most pristine ecosystems, largely preserved from anthropogenic impacts due to their geographical isolation and a historically very limited human occupation (Le Gall et al., 1985; Barré and Servan, 1988; Le Corre and Jouventin, 1997; Le Corre and Probst, 1997; Probst et al., 2000; Le Corre and Safford, 2001; Probst et al., 2001; Durville et al., 2003; Boullet, 2005; Chabanet and Durville, 2005; Boullet, 2006; Gravier-Bonnet and Bourmaud, 2006a; Gravier-Bonnet and Bourmaud, 2006b; Quod et al., 2007; Boullet, 2008a; Boullet, 2008b; Bourjea et al., 2011; Poupin et al., 2013a; Poupin et al., 2013b; Boullet, 2014; Dumeau et al. 2014; Chabanet et al., 2016; Conand et al., 2016; Poupin, 2016; Quétel et al., 2016). The Iles Eparses are among the last sanctuaries of marine and terrestrial tropical biodiversity in the southwest Indian Ocean (Quétel et al., 2016).

Limited to a few temporary occupations by fishermen for a long time, human impact on these islands mostly grew towards the end of the $19^{\text {th }}$ century and during the first half of the $20^{\text {th }}$ century with the development of agricultural (production of copra, sisal, etc.) and industrial (phosphate mines) activities, particularly on Grande Glorieuse and Juan de Nova, which were respectively exploited for 73 years (from 1885 to 1958) and 75 years (1900-1975). These activities, which proved unprofitable in an insular context, were abandoned during the second half of the $20^{\text {th }}$ century (Hoareau, 1993; Caceres, 2003). Europa was relatively spared by human presence, due to the hostile living conditions (no fresh water, massive presence of mosquitoes, arid climate). A few rare attempts of settlement took place around 1860 and continued in the early $20^{\text {th }}$ century, with the introduction of goats, sisal and green choca, but they did not last long and all ended in failures (Hoareau, 1993; Caceres, 2003). Finally, Tromelin, given its reduced dimensions, its isolation and its extreme living conditions, was never voluntarily colonized or exploited. We can nevertheless note the episode of the shipwrecked victims of 
the Utile, a French boat, from which a hundred or so Malagasy slaves were abandoned on the island in 1761. Eight survivors were rescued by the Chevalier de Tromelin in 1774 (Frain, 2009; Hoareau, 1993). Meteorological stations were installed in the 1950's on each of these islands along with the presence of Météo-France agents for 6 month periods, followed in the 1970's by the settlement of military units on Europa, Juan de Nova and Grande Glorieuse, on rotation every 45 days. These periods are those of the first durable constructions on these islands: buildings and landing strips (Hoareau, 1993; Caceres, 2003).

Given their protected status that prohibits human disruption, and the strict regulations regarding their access (TAAF, 2011), although each island presents specific ecological characteristics and conservation stakes (linked to the impact of their past exploitation by men), they represent control areas for scientific studies in physical and life sciences (Cointat, 2010). However, the implementation of strategies and conservatory management actions seem necessary in order to reduce increasing anthropogenic disruption.

\section{History of Botanic Studies}

The first botanic notes go back to the second half of the $19^{\text {th }}$ century with Speke's passing through in 1859 on Europa (Speke, 1863), Coppinger's (1882) then Abbott's (1893) on the Iles Glorieuses. During the $20^{\text {th }}$ century, around twenty scientists made their contribution to the inventory of the vascular flora of the Iles Eparses (a «*» indicates a contribution based solely on the examination of herbarium material), especially Europa's (Voeltzkow, 1904; Perrier de La Bâthie, 1921; Poisson, 1923; Decary, 1937; Bosser*, 1952; Paulian, 1955; Capuron, 1966; Battistini, 1966; Delépine et al., 1976; Le Corre and Jouventin, 1997) and, to a lesser extent, Juan de Nova's (Perrier de La Bâthie, 1921; Decary, 1937; Bosser*, 1952), the Iles Glorieuses' (Battistini and Cremers, 1972; Cadet, 1984) and Tromelin's (Paulian, 1955; Staub, 1970). However, their stay was often short, not exceeding a few days, and most of them were naturalists or zoologists.

At the beginning of the 21 st century, the botanical knowledge of these territories showed a significant increase particularly due to the Conservatoire Botanique National de Mascarin's involvement (thereafter CBNM) with several expeditions to study the flora and plant communities (Table 1).

\section{Objective}

The objective of this article is to provide an updated state of the terrestrial vascular flora (Tracheophytes) for the four island groups of the Iles Eparses supporting terrestrial vegetation, namely Europa, Juan de Nova, Iles Glorieuses and Tromelin (Bassas da India lacking terrestrial vegetation).

\section{MATERIAL AND METHODS}

\section{Taxonomic Identification of Plant Material and Nomenclature}

The determinations were made in situ from the observation of fresh material or, in the case of taxa requiring a more in-depth study, herbarium specimens - within the herbaria of the CBNM in La Réunion [CBNM], and/or in Paris within both the Missouri Botanical Garden [MO] and the Muséum National d'Histoire Naturelle [P] - and/or plants parts in alcohol (deposited at the CBNM). On a more anecdotal level and according to opportunities, information was also gathered from people who, in the past, had long stays on the Iles Eparses (Météo-France staff mostly). These interviews made it possible to corroborate alien taxa which benefited from an attempted plantation but didn't survive. 
Table 1. List of the field trips of the CBNM on the Iles Eparses between 2004 and 2017.

\begin{tabular}{|c|c|c|c|}
\hline Territory & Date & $\begin{array}{c}\text { Duration } \\
\text { (days) }\end{array}$ & Staff \\
\hline Iles Glorieuses & March 2004 & 2 & Boullet \\
\hline Iles Glorieuses & August 2005 & 7 & Boullet \\
\hline Europa & May - June 2006 & 11 & Boullet \\
\hline Juan de Nova & October - November 2009 & 12 & Boullet, Hivert, Lacoste \\
\hline Europa & May 2011 & 4 & Boullet, Gigord, Hivert \\
\hline Juan de Nova & May 2011 & 4 & Boullet, Gigord, Hivert \\
\hline Iles Glorieuses & May 2011 & 4 & Fontaine, Hivert \\
\hline Tromelin & May 2011 & 1 & Fontaine, Hivert \\
\hline Europa & October - December 2011 & 52 & Dumeau, Hivert \\
\hline Europa & March 2012 & 2 & Gigord, Hivert \\
\hline Iles Glorieuses & May - June 2012 & 19 & Beaurepaire, Férard, Gigord, Hivert \\
\hline Europa & September 2012 & 2 & Hivert \\
\hline Europa & October 2012 & 2 & Gigord, Lavergne \\
\hline Europa & February 2013 & 2 & Hivert \\
\hline Juan de Nova & March 2013 & 24 & Férard, Fontaine, Hivert \\
\hline Tromelin & August - September 2013 & 14 & Fontaine, Gigord \\
\hline Europa & December 2013 & 2 & Hivert \\
\hline Iles Glorieuses & January - March 2014 & 48 & Dufour, Hivert \\
\hline Europa & July 2014 & 2 & Hivert \\
\hline Europa & December 2014 & 2 & Hivert \\
\hline Europa & April 2015 & 2 & Chauvrat, Hivert \\
\hline Europa & November 2015 & 2 & Hivert \\
\hline Iles Glorieuses & November 2015 & 3 & Hivert \\
\hline Europa & May - July 2016 & 61 & Boullet, Hivert \\
\hline Tromelin & December 2016 & 1 & Gigord, Hivert \\
\hline Tromelin & February 2017 & 1 & Fossy, Hivert, Picot \\
\hline Europa & June 2017 & 2 & Hivert \\
\hline Iles Glorieuses & August - September 2017 & 30 & Anxionnaz, Boullet, Hivert \\
\hline Tromelin & October 2017 & 1 & Rochier \\
\hline Tromelin & November 2017 & 10 & Boullet, Gigord, Hivert \\
\hline
\end{tabular}

Taxonomic identification was based on standard flora related to the western Indian Ocean: Flore of Madagascar and the Comores (Humbert and Leroy, 1936-2001), Flora of Tropical East Africa (Polhill, 1948-2012), Flora Zambesiaca (Pope et al., 1960-2005), Flora of the Seychelles (Friedmann, 1994), Flora of Aldabra and neighboring islands (Fosberg and Renvoize, 1980), Flora of the Mascarenes (Bosser et al., 1976-2009), The Catalogue of the Vascular Plants of Madagascar (Madagascar Catalogue, 2017) and Flora of Somalia (Thulin, 1993-2006).

In general, for the whole flora, systematic treatment took most recent evolution of the phylogenetic nomenclatures into account. For angiosperms, the preferred treatment is the one proposed by The Angiosperm Phylogeny Group (2009) or by the 'Angiosperm Phylogeny Website' (Stevens, 2001 2015). For the Lycophytes and the Ferns, we followed Christenhusz et al. (2011), which relies in particular on the phylogenetic studies of Pryer et al. (2004), and Schuettpelz and Pryer (2007). Finally, in the case of taxa also present on Reunion Island and/or on Mayotte, indexes of vascular flora of Mayotte (CBNM, 2013b) and of vascular flora of Reunion Island (CBNM, 2017), the latter being included in TAXREF (French National Referential), serve as taxonomic and nomenclatural references. Regarding the citation of authorities, the nomenclature got homogenized according to the international standard author abbreviations by Brummit and Powell (1992) whose updating is on-going within the framework of IPNI (The International Plant Name Index, 2017).

All specimens collected were taxonomically identified to species or inferior ranking i.e. subspecies, varietas (variety) or form. By taxa we mean different taxonomic entities at species level or inferior 
rank. In rare cases, some taxa were solely determined to the family or genus rank, adding «sp. » sometimes followed by a number of order (e.g. Phyllanthus sp.2).

\section{Chorology and Origin of Taxa}

Two types of data were compiled in order to quantify the floristic diversity of the Iles Eparses: older floristic reports coming from bibliography (before 2004, date of the first inventories of the CBNM) and recently confirmed reports (period 2004-2017, based on field surveys by the CBNM). Older floristic reports were evaluated according to the observer's competence field, date of observation and general coherence, and were compared with recently confirmed floristic reports. After this evaluation, all dubious literature records for taxa not found on the Iles Eparses during the period 20042017 were ignored.

Lists of taxa specific to each territory (Europa, Juan de Nova, Iles Glorieuses and Tromelin) will be presented according to the following fields: botanical name, family, general status (origin status on a given territory on the base of chorological, ecological and biological data defined in three statuses according to the terms defined by Pyšek et al. (2004), Mitić et al. (2008) and Carlton (1996): native $[\mathrm{N}]$ (taxa naturally found on the territory considered, independently of any human role); cryptogenic $[\mathrm{K}]$ (taxa without certainty of native or alien status to the region); alien [A] (species originating from a region located outside its natural distribution area and introduced deliberately or accidentally by humans or arriving naturally outside its natural distribution area), population status following the scale defined by Richardson et al. (2000) (not naturalized, naturalized [sensu lato, including naturalized and casual alien plants], invasive or native possibly supplemented by the mention 'cultivated' for cultivated taxa) and the distribution range reflecting the area of occupancy of the taxa on the Iles Eparses: locally naturalized (taxa still present on the territory but confined to a small geographical scale), widely naturalized (taxa still present on the territory on a large scale) and presumed extinct (taxa found with certainty but that have apparently disappeared because not found during recent inventories).

Comparative analyses between territories focused on floristic diversity and general status, then on the biogeography of taxa considered to be native. Altogether, 9 chorological categories were used: strict endemic (endemic in a single island); Madagascar (sensu stricto); western Indian Ocean (biogeographical areas corresponding to the territories located in the west of the Indian Ocean such as the Mascarenes, Madagascar, the Comoros and the Seychelles); Africa (African continent); IndoPacific (biogeographical area including the tropical and subtropical areas of the Indian Ocean and the occidental part of the Pacific Ocean); paleotropical (biogeographical intertropical area of the ancient world: Africa, Asia, Pacific); pantropical (biogeographical area corresponding to all the tropical areas of the globe); cosmopolitan (taxa with a very vast geographical distribution and spread almost worldwide); unknown (in the case of undetermined taxa).

\section{RESULTS}

\section{Overall Assessment of the Terrestrial Vascular Flora of the Iles Eparses}

Between 2004 and 2017 botanists of the CBNM spent a total of 706 days on field expeditions to the Iles Eparses (Table 1). These inventories made it possible to significantly improve the knowledge related to the floristic diversity, until then largely underestimated on all the territories (Figure 6 and Figure 7). 


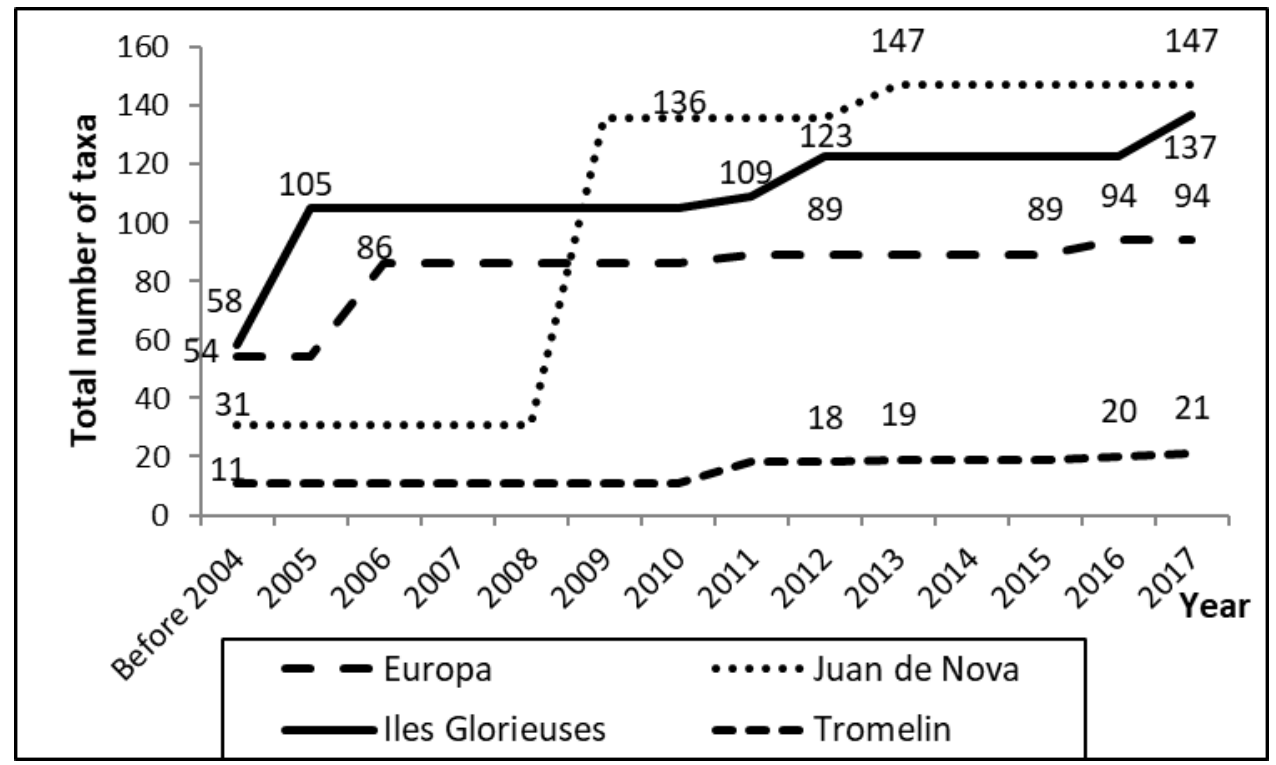

Figure 6. Cumulative number of taxa reported for the Iles Eparses through time.

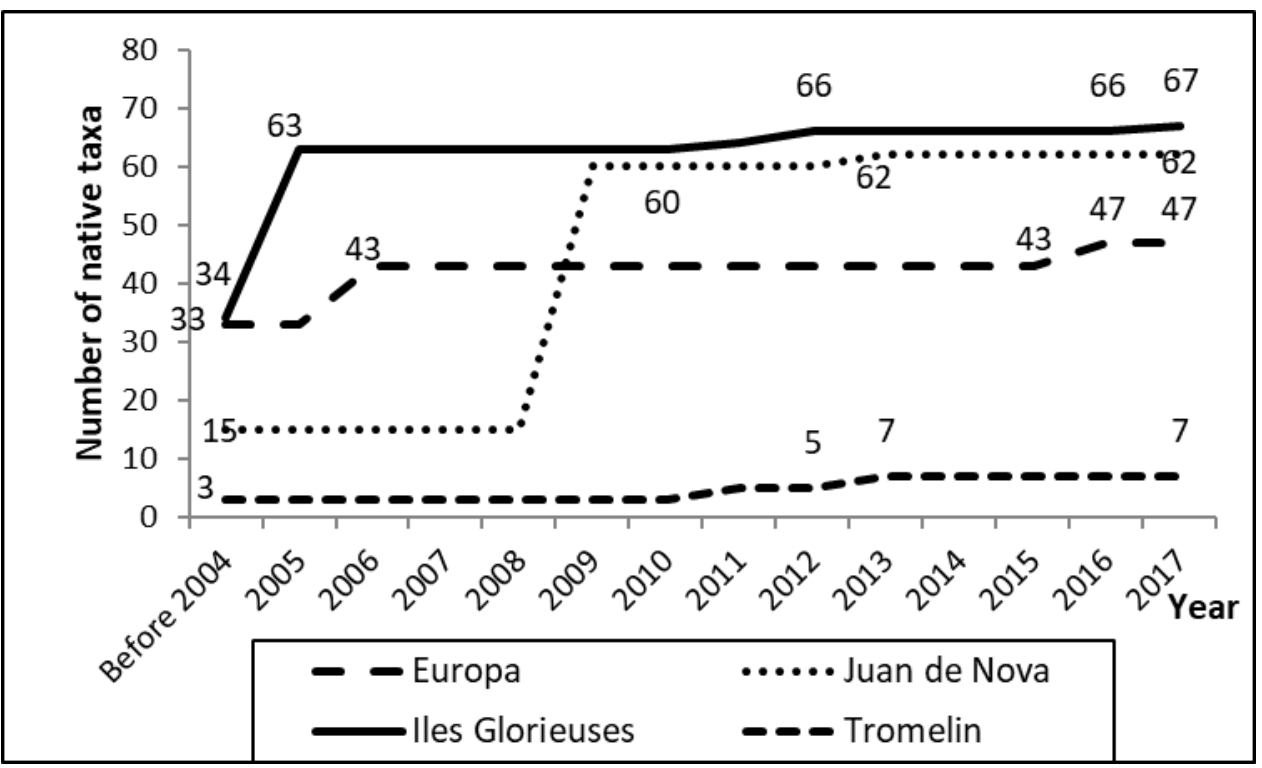

Figure 7. Cumulative number of native taxa reported for the Iles Eparses through time.

Figure 6 shows increases of approximately:

- $\quad+74 \%$ for Europa's flora (from 54 listed taxa before 2004 to 94 in 2016)

- $\quad+374 \%$ for Juan de Nova's flora (from 31 taxa to 147 in 2013)

- $\quad+136 \%$ for the Iles Glorieuses' flora (from 58 taxa to 137 in 2017)

- $\quad+91 \%$ for Tromelin's flora (from 11 taxa to 21 in 2017)

The same analyses made on strict native taxa for each territory (Figure 7) show similar patterns with increases of approximately:

- $\quad+42 \%$ for Europa's native flora (from 33 listed taxa before 2004 to 47 in 2016)

- $\quad+313 \%$ for Juan de Nova's native flora (from 15 taxa to 62 in 2013)

- $\quad+97 \%$ for the Iles Glorieuses' native flora (from 34 taxa to 67 in 2017)

- $\quad+133 \%$ for Tromelin's native flora (from 3 taxa to 7 in 2013) 
As a consequence, these results are comparable whatever the status of the flora considered and allow us to rule out the hypothesis of a massive and recent introduction of alien taxa and possibly cryptogenic taxa that could have explained such a significant increase in floristic diversity.

The global list of all the taxa found on the Iles Eparses is presented in Table 2 (Appendix A). In the current state of knowledge, 250 taxa were listed on the whole of the Islands (120 native, 107 alien and 23 cryptogenic). They concern 66 botanical families, 178 genera, 250 species, 7 subspecies, 16 varieties and 1 form. The richest families in taxa are Poaceae (38 taxa), Fabaceae and Malvaceae (18 taxa), Amaranthaceae (15 taxa) and Euphorbiaceae (11 taxa). The richest genera in taxa are Euphorbia (8 taxa), Achyranthes and Boerhavia (6 taxa), Dactyloctenium, Eragrostis, Phyllanthus and Portulaca (5 taxa).

As shown in Figure 6, Figure 7 and Table 2 (Appendix A), most taxa were mentioned for the first time only after the start of fieldwork inventories by the botanists of the CBNM in 2004: among the 399 taxa mentioned, 157 (39\%) have not been first mentioned by the CBNM (but verified in the field) and only $12(3 \%)$ have never been observed by the CBNM (e.g., alien species formerly cultivated and extinct before 2004 but reported in the literature). Some old citations have required much effort, focused investigation and validation in the field given the uncertainty/imprecision of the data collected in the literature.

\section{Assessments and Floristic Analyses per Territory}

The detailed lists of taxa per territory are presented in Tables 3 to 6 (Appendix B).

\section{Floristic Diversity and General Status}

In Table 7, in terms of absolute value, all taxa combined, Tromelin has the lowest plant diversity while Juan de Nova has the largest diversity in terms of numbers of species.

Table 7. Assessment of the total number of taxa and their general status per territory and in the study area.

\begin{tabular}{|c|c|c|c|c|c|}
\hline Iles Eparses (land area) & $\begin{array}{l}\text { Total number } \\
\text { of families }\end{array}$ & $\begin{array}{c}\text { Total number } \\
\text { of taxa }\end{array}$ & $\begin{array}{c}\text { Number of native } \\
\text { taxa (\%) }\end{array}$ & $\begin{array}{c}\text { Number of } \\
\text { cryptogenic taxa } \\
(\%)\end{array}$ & $\begin{array}{c}\text { Number of alien } \\
\text { taxa (\%) }\end{array}$ \\
\hline Europa $\left(30 \mathrm{~km}^{2}\right)$ & 35 & 94 & $47(50 \%)$ & $8(9 \%)$ & $39(41 \%)$ \\
\hline Juan de Nova $\left(5 \mathrm{~km}^{2}\right)$ & 49 & 147 & $62(42 \%)$ & $16(11 \%)$ & $69(47 \%)$ \\
\hline Iles Glorieuses (4.37 km²) & 53 & 137 & $67(49 \%)$ & $9(7 \%)$ & $61(44 \%)$ \\
\hline Grande Glorieuse $\left(4.2 \mathrm{~km}^{2}\right)$ & 52 & 133 & 65 (49\%) & $8(6 \%)$ & $60(45 \%)$ \\
\hline Ile aux Crabes $\left(0.005 \mathrm{~km}^{2}\right)$ & 5 & 6 & $4(67 \%)$ & $1(16.5 \%)$ & $1(16.5 \%)$ \\
\hline Ile du Lys $\left(0.14 \mathrm{~km}^{2}\right)$ & 17 & 27 & $22(82 \%)$ & $2(7 \%)$ & $3(11 \%)$ \\
\hline Ile aux Roches Vertes $\left(0.025 \mathrm{~km}^{2}\right)$ & 4 & 4 & $4(100 \%)$ & 0 & 0 \\
\hline Tromelin $\left(1 \mathrm{~km}^{2}\right)$ & 18 & 21 & $7(33 \%)$ & 0 & $14(67 \%)$ \\
\hline TOTAL & 66 & 250 & $120(48 \%)$ & $23(9 \%)$ & 107 (43\%) \\
\hline
\end{tabular}

If we compare the floristic diversity specific to each of the Iles Glorieuses (Table 7), it is found that Grande Glorieuse has the greatest number of taxa (133) but one of the lowest percentages of native species (49\%). The other islands composing the Iles Glorieuses have a smaller surface area and a low floristic diversity but a relatively high percentage of native species $(67 \%$ to $100 \%)$.

By weighting the values of the native flora in relation to the terrestrial surface (in $\mathrm{km}^{2}$ ) of each island, we see that Europa presents the lowest ratio of native taxa $/ \mathrm{km}^{2}\left(1.56 / \mathrm{km}^{2}\right)$, followed by Tromelin $\left(7 / \mathrm{km}^{2}\right)$, while Juan de Nova and the Iles Glorieuses present the highest ratio of native taxa $\left(12.4 / \mathrm{km}^{2}\right.$ and $15.3 / \mathrm{km}^{2}$, respectively). 
In Table 8, the chorological analysis of the native vascular flora of Europa, Juan de Nova and the Iles Glorieuses reveals that between $24 \%$ and $30 \%$ of the taxa have a pantropical distribution. Their native flora is also characterized by a relatively important contribution of taxa with Indo-Pacific range (19\% and $13 \%$ for Europa and Juan de Nova, respectively), in particular in the case of the Iles

Glorieuses (22\%). In the same way, the native flora of these three islands is clearly marked by the contribution of taxa which are confined to the western Indian Ocean (24\% for Europa and Juan de Nova), a feature that is particularly true for the Iles Glorieuses (30\%). On the other hand, it is clear that the native flora of Europa and to a lesser extent that of Juan de Nova are strongly influenced by the contribution of the Malagasy flora sensu stricto (respectively 15\% and 13\%), which is not the case for the Iles Glorieuses (1.5\%).

The chorological analysis of Tromelin's flora provides less information due to the presence of only seven native taxa with a large geographical range (14.5\% pantropical, $42 \%$ Indo-Pacific range, $14.5 \%$ western Indian Ocean).

Table 8. Chorology of the native vascular flora per territory of the Iles Eparses.

\begin{tabular}{|c|c|c|c|c|c|c|c|c|}
\hline \multirow[b]{2}{*}{ Chorological type } & \multicolumn{2}{|c|}{ Europa } & \multicolumn{2}{|c|}{ Juan de Nova } & \multicolumn{2}{|c|}{$\underline{\text { Iles Glorieuses }}$} & \multicolumn{2}{|c|}{ Tromelin } \\
\hline & $\begin{array}{c}\text { Number of } \\
\text { taxa }\end{array}$ & $\%$ & $\begin{array}{c}\text { Number of } \\
\text { taxa }\end{array}$ & $\%$ & $\begin{array}{c}\text { Number of } \\
\text { taxa }\end{array}$ & $\%$ & $\begin{array}{c}\text { Number of } \\
\text { taxa }\end{array}$ & $\%$ \\
\hline Strict endemic & 2 & $4 \%$ & 1 & $1.5 \%$ & 0 & $0 \%$ & 1 & $14.5 \%$ \\
\hline Madagascar (sensu stricto) & 7 & $15 \%$ & 8 & $13 \%$ & 1 & $1.5 \%$ & 0 & $0 \%$ \\
\hline Western Indian Ocean & 11 & $24 \%$ & 15 & $24 \%$ & 20 & $30 \%$ & 1 & $14.5 \%$ \\
\hline Africa & 1 & $2 \%$ & 1 & $1.5 \%$ & 0 & $0 \%$ & 0 & $0 \%$ \\
\hline Indo-Pacific & 9 & $19 \%$ & 8 & $13 \%$ & 15 & $22 \%$ & 3 & $42 \%$ \\
\hline Paleotropical & 3 & $6 \%$ & 8 & $13 \%$ & 8 & $12 \%$ & 0 & $0 \%$ \\
\hline Pantropical & 11 & $24 \%$ & 16 & $26 \%$ & 20 & $30 \%$ & 1 & $14.5 \%$ \\
\hline Cosmopolitan & 1 & $2 \%$ & 2 & $3 \%$ & 2 & $3 \%$ & 1 & $14.5 \%$ \\
\hline Unknown & 2 & $4 \%$ & 3 & $5 \%$ & 1 & $1.5 \%$ & 0 & $0 \%$ \\
\hline
\end{tabular}

Europa, Juan de Nova and Tromelin host possibly strict endemic species: morphotypes of the genera Achyranthes (Achyranthes sp. nov., Amaranthaceae) and Euphorbia (Euphorbia sp. nov., Euphorbiaceae), which are not reportable to species described and whose current study suggests that they are peculiar to Europa; Hypoestes juanensis Benoist (Acanthaceae) on Juan de Nova and Boerhavia sp. nov. 2 on Tromelin. We also note the presence of an endemic taxon on the scale of the Mozambique channel, Perrierophytum glomeratum Hochr. (Malvaceae), which grows on Juan de Nova and on the Grande Glorieuse and another one called Boerhavia sp. nov.1 which is present on Juan de Nova and on the Iles Glorieuses.

\section{Population Status, Distribution Range and Cultural Status of the Alien Flora}

Europa, Juan de Nova and the Iles Glorieuses have a high proportion of naturalized (sensu lato) alien taxa, ranging from 58\% (Juan de Nova) to $85 \%$ (Iles Glorieuses; Table 9). In contrast, the alien flora of Tromelin is made up of $50 \%$ of non-naturalized taxa. It should also be noted that each territory holds a significant proportion (between $6 \%$ and $36 \%$ ) of invasive alien taxa. 
Table 9. Assessment of the population status, distribution range and cultural status of the alien vascular flora per territory of the Iles Eparses.

\begin{tabular}{lccc|ccc|ccc}
\hline Iles Eparses & \multicolumn{3}{c|}{ Population status } & \multicolumn{2}{c|}{ Distribution range } & \multicolumn{3}{c}{ Cultural status } \\
& $\begin{array}{c}\text { Not naturalized } \\
\text { (\%) }\end{array}$ & $\begin{array}{c}\text { Naturalized } \\
\text { sensu lato (\%) }\end{array}$ & $\begin{array}{c}\text { Invasive } \\
\text { (\%) }\end{array}$ & $\begin{array}{c}\text { Supposed } \\
\text { extinct (\%) }\end{array}$ & $\begin{array}{c}\text { Local } \\
\text { (\%) }\end{array}$ & $\begin{array}{c}\text { Wide } \\
\text { (\%) }\end{array}$ & $\begin{array}{c}\text { Not cultivated Cultivated on a Cultivated on a } \\
\text { (\%) }\end{array}$ & $\begin{array}{c}\text { small scale (\%) } \\
\text { sarge scale (\%) }\end{array}$ \\
\hline Europa & $6(15 \%)$ & $30(77 \%)$ & $3(8 \%)$ & $7(18 \%)$ & $30(77 \%)$ & $2(5 \%)$ & $27(69 \%)$ & $9(23 \%)$ & $3(8 \%)$ \\
Juan de Nova & $25(36 \%)$ & $40(58 \%)$ & $4(6 \%)$ & $4(6 \%)$ & $56(81 \%)$ & $9(13 \%)$ & $30(44 \%)$ & $36(52 \%)$ & $3(4 \%)$ \\
Iles Glorieuses & $2(3 \%)$ & $52(85 \%)$ & $7(12 \%)$ & $4(7 \%)$ & $49(80 \%)$ & $8(13 \%)$ & $44(72 \%)$ & $15(25 \%)$ & $2(3 \%)$ \\
Tromelin & $7(50 \%)$ & $2(14 \%)$ & $5(36 \%)$ & $7(50 \%)$ & $7(50 \%)$ & 0 & $4(29 \%)$ & $10(71 \%)$ & 0 \\
\hline
\end{tabular}

Analyses of 'distribution range' indicate that the majority of alien taxa are naturalized only locally on Europa, Juan de Nova and Iles Glorieuses (between $77 \%$ and $81 \%$ ), while a small proportion of these taxa are naturalized on a large scale (between 5\% and 13\%). Some taxa mentioned in the past have not been recently confirmed for these territories (between 6\% and 18\%), and are considered as 'supposedly extinct'. The same analysis for Tromelin provides different results: a high proportion $(50 \%)$ of alien taxa introduced by humans has not survived through time. Some of these taxa have, however, naturalized locally (50\%).

The analysis of the cultural status of the alien flora shows two tendencies: while the alien flora of Europa and the Iles Glorieuses is mostly made up of non-cultivated taxa (69\% and 72\%, respectively), the flora of Juan de Nova and Tromelin contain a high proportion of cultivated taxa on small and/or large scales (56\% and $71 \%$, respectively). A more detailed analysis of the taxa that have been subject to plantations on a small scale reveals that the main motivation on Europa, Juan de Nova and Tromelin was the introduction of ornamental plants and, secondly, the introduction of food plants. On Grande Glorieuse, the only island of the atoll subject to plantations, the main intention was to introduce food plants and secondly ornamental plants. In the case of alien taxa cultivated on a large scale, which is the case for Europa, Juan de Nova and the Iles Glorieuses, the main objective was to introduce taxa that could provide agricultural products. Finally, some native species were cultivated on Juan de Nova for their ornamental value, and alien food species are cultivated in pots on Tromelin.

\section{DISCUSSION}

\section{Overview of the Flora of the Iles Eparses}

The knowledge of the terrestrial vascular flora of the Iles Eparses has considerably improved since 2004, thanks to the plant inventories led by the CBNM. This result clearly demonstrates knowledge gaps on these territories during the $19^{\text {th }}$ and $20^{\text {th }}$ centuries, especially on Juan de Nova, the Iles Glorieuses and Tromelin, which, unlike Europa, had until recently the reputation of being floristically poor and with little heritage value (Caceres, 2003).

The diversity of the Iles Eparses (120 native, 107 alien and 23 cryptogenic, 66 families altogether) can be compared to that listed for the Aldabra group (Aldabra, Cosmoledo, Assumption and Astove), located in the southwest of the Seychelles archipelago, where Fosberg and Renvoize (1980) and Friedmann $(1994,2012)$, excluding the seagrass taxa, reported 265 species, subspecies and varieties in 78 families (about 166 can be considered as native taxa). The Iles Eparses nevertheless have a total terrestrial surface area close to $40.37 \mathrm{~km}^{2}$ (an average of 2.97 native taxa $/ \mathrm{km}^{2}$ ) while the Aldabra group comes to a terrestrial surface area close to $156 \mathrm{~km}^{2}$ according to Gibson and Phillipson (1983) (an average of 1.06 native taxa $/ \mathrm{km}^{2}$ ). The diversity of species on the Iles Eparses could be due to the fact that the Iles Eparses are scattered across a larger latitudinal range, and thus encompass a wider range of ecological conditions. However, the rate of endemism on a regional scale is notably higher in the Aldabra island group, where it reaches 20 endemic taxa (12\% of the global floristic diversity), than in the Iles Eparses where only 6 hypothetic endemic taxa have been reported (Achyranthes sp. nov. and Euphorbia sp. nov. on Europa, Hypoestes juanensis on Juan de Nova, Boerhavia sp. nov.1 and Perrierophytum glomeratum on Juan de Nova and Iles Glorieuses, Boerhavia sp. nov.2 on Tromelin). 
The rate of endemism in the Aldabra group could be explained by the fact that Aldabra is by far the largest atoll, the group contains a higher number of islands in the region, and that such atolls are also relatively scarce in the southwest Indian Ocean (excluding the Seychelles).

If we compare the native floristic diversity of the Aldabra group with that of the Iles Glorieuses (Boullet, 2005), these two groups being closely related biogeographically, we note a strong floristic similarity (more than $90 \%$ of the native taxa found on Iles Glorieuses is also found on Aldabra). Both groups have common endemic species such as Bulbostylis basalis Fosberg, Euphorbia stoddartii Fosberg and Portulaca mauritiensis Poelln. var. aldabradensis Fosberg (this taxon being also found in Tromelin). The endemism of the Aldabra group can be explained by the number of atolls (four for the Aldabra group versus one for the Iles Glorieuses), as well as the type and the diversity of these atolls (3 raised atolls, among which Aldabra is one of the largest atoll known in the world (Goldberg, 2016; Hnatiuk, 1979), while Iles Glorieuses are an immersed atoll composed of a large sandy isle and few remnants of emerged coral reefs). In addition, the total surface area of emerged land is $\sim 156 \mathrm{~km}^{2}$ for the Aldabra group (Gibson and Phillipson, 1983) versus $\sim 4 \mathrm{~km}^{2}$ for the Iles Glorieuses, i.e., 39 times less, the diversity of habitats due to the morphology of the atolls and, on the Iles Glorieuses, the rarity or absence of specific habitats hosting a high number of endemic species such as tortoise pastures, and the xerophytic mixed scrub on reef limestone (Fosberg 1971) are factors in endemism.

\section{Assessment of the Flora of the Iles Eparses}

\section{Floristic Diversity}

Although the raw values of floristic diversity for each territory indicate that Juan de Nova possesses the largest number of taxa while Tromelin shelters the lowest number, when these values are weighted by the terrestrial surface area of each territory the following gradient of florisitic diversity (either for all taxa, or for native taxa) can be observed: Europa $<$ Tromelin $<$ Juan de Nova $<$ the Iles Glorieuses, with the latter two being close.

This gradient can be explained by the climatic conditions and the edaphic constraints specific to each territory (Delépine et al., 1976; Caceres, 2003). Europa (Figure 2) has a subarid climate with a long and marked dry season (April to October), low precipitation $(541.2 \mathrm{~mm} /$ year on average with a minimum close to $0 \mathrm{~mm}$ per month), strongly saline skeletal soils, as well as Tromelin (Figure 5) which, although subject to a tropical maritime climate with a less marked dry period (September to November) and moderate to high precipitation (1105.5 mm/year average; $30.2 \mathrm{~mm} / \mathrm{month}$ minimum), is largely subjected to a strong influence of swell and salinity due to its very small dimensions and the absence of a protective coral reef barrier. The low floristic diversity of Tromelin is likely the consequence of several combined factors: the reduced surface area of the island, its flat morphology with a high exposure to salt and recurrent heavy swell, its isolation in the mid-west Indian Ocean, not much suitable for colonization by propagules or diaspores, and its position in the middle of most cyclone routes. In contrast, the Iles Glorieuses and Juan de Nova present more favorable situations for the development of vegetation. The Iles Glorieuses (Figure 4), located on the southern limit of low equatorial pressures, are subject to two distinct seasons, a fresh season from May to November marked by a short period of dryness (September to November) and a warm season from December to April marked by important precipitation ( $1078.3 \mathrm{~mm} /$ year average; $11.4 \mathrm{~mm} / \mathrm{month}$ minimum) and high level of humidity $(81 \%-84 \%)$. The influence of salinity seems weaker compared with Europa and Tromelin (Delépine et al., 1976). On Juan de Nova (Figure 3), the climate is also characterized by two seasons: a fresh season from April to November during which rainfall is very low (1.9 to 37.6 $\mathrm{mm} / \mathrm{month}$ ) resulting in a marked period of dryness, and a rainy season from December to March during which total precipitation ranges from $100.5 \mathrm{~mm}$ to $282.1 \mathrm{~mm}$. We also not that the average sunshine duration slowly increases from Tromelin ( 8.1 hours/ day) to the Iles Glorieuses ( 8.2 hours/day) and Europa (9 hours/day) (Delépine et al., 1976; Météo-France, 2016).

Apart from the particular case of the isolated Tromelin island, these results are in accordance with the principle of increasing floristic diversity with increasing precipitation, and to some extent, decreasing latitude, a principle also described in the "Fosberg zones" (Fosberg, 1956; Stoddart, 1992). These zones have been defined for the atolls of the Pacific for which the floristic diversity is positively 
correlated with the mean annual rainfall but not with the size/surface area of the islands or with the distance between the island considered and other larger territories. Moreover, it has been proved that vegetation responds asymmetrically to extreme precipitation because vegetation is far more sensitive to dryness than to humidity (Mueller-Dombois and Fosberg, 1998), as it is for instance the case for Europa. Finally, another parameter that strongly influences floristic diversity is temperature, especially the actual quantity of heat received. It is therefore logical to observe an increase in biodiversity in the latitudinal gradient from Europa to the Iles Glorieuses.

On the other hand, we can't compare our results with those of Larrue et al. (2015) which support the view that the spatial pattern of native species richness observed on Eastern Polynesia's remote atolls was strongly influenced by (i) atoll area but also by (ii) the distance to the raised atoll of Makatea, and (iii) the distance to the volcanic island of Tahiti. Indeed, we don't think these Pacific considerations can be applied to the isolated atolls of the Mozambique Channel. While it is clear that the size of islands plays a role as well as their age, it is above all the bioclimatic context and the supply of habitats that influence the specific diversity.

\section{Origin of the Native Flora}

The chorological analysis showed that the native flora typical of Europa, Juan de Nova and the Iles Glorieuses is largely made of pantropical and indo-pacific taxa and taxa from islands of the western Indian Ocean. The latter influence is particularly marked for the Iles Glorieuses which, by their geographical position and the influence of a zone of weak currents (Ocean Surface Current Analyses, 2015), have been naturally colonized through time by taxa coming from nearby islands such as the Comoros or the islands of the Seychelles. The north-west tip of Madagascar may have little influence on the native flora of the Iles Glorieuses since a single Malagasy taxon (Ficus grevei Baillon), likely dispersed by birds, is common to the two territories.

In contrast, Europa and Juan de Nova are more influenced by the Malagasy flora sensu stricto, which can be explained by the relative geographic proximity of these territories, by their environmental conditions close to the sub-arid coastal regions of the South of Madagascar and by the strong influence of the sea currents in the Mozambique channel, North-flowing and reaching the occidental coast line of Madagascar (Ocean Surface Current Analyses, 2015). The analysis of dispersal modes of some native Malagasy taxa sensu stricto found on Europa and/or Juan de Nova (but not found on the Iles Glorieuses) allows us to implicate the role of the ocean (i.e., the main dispersal vector for Salsola littoralis Moq.), the role of the wind (the likely dispersal vector for Cynanchum luteifluens (Jum. \& H. Perrier) Desc. and Psiadia altissima (DC.) Drake) and the role of bird-assisted dispersal (e.g., for Cleome grandidieri Baill., Cheirolaena linearis Benth, Dactyloctenium capitatum A. Camus, Dombeya greveana Baill. var. metameropsis (Hochr.) Arènes, Eragrostis capuronii A. Camus, Euphorbia stenoclada Baill., Ficus marmorata Bojer ex Baker, Nesogenes madagascariensis (Bonati) Marais, Plumbago aphylla Boj. ex Boiss. and Salvadora angustifolia Turrill) on the colonization of these two islands of the Mozambique channel to respective distances of 300 and 140 $\mathrm{km}$ west of Madagascar.

The chorological analysis of the native flora of Tromelin, although not very significant regarding the small number of native taxa $(n=7)$, shows that it is mostly made of taxa with large ranges (cosmopolitan, pantropical and indo-pacific types). Indeed, located outside the Mozambique Channel, Tromelin is under the influence of the subequatorial current, very stable and west-flowing all through the year (Ocean Surface Current Analyses, 2015). The native flora of Tromelin is also marked by few taxa confined to the western Indian Ocean, demonstrating the significant influence of the nearby Seychelles islands.

The strict endemism rates are particularly low in the Iles Eparses. This result is explained by the fact that they are young oceanic islands, geographically close to huge biodiversity reservoirs with comparable physical and abiotical conditions. Moreover, according to the available data (Camoin et al., 1984; Camoin et al., 1997; Virah-Sawmy et al., 2009; Woodroffe and Horton, 2005) the sea level has changed considerably since the likely formation of the Iles Eparses during the peak of the last interglacial, Marine Isotope Stage 5 (MIS5, 120,000 years ago). The islands probably peaked at a height of 120-140 m above sea level at the Last Glacial Maximum (LGM, 25,000 years ago).

Deglacial sea levels rose irregularly in stages to present, with a maximum oscillation at about 7000- 
6000 years BP, to about 1-3 m higher than present sea level (Camoin et al., 1984; Camoin et al., 1997). At that time Europa, Juan de Nova and Grande Glorieuse were not entirely submerged, but Tromelin undoubtedly and the Ile du Lys probably were.

Yet some particular cases deserve to be noted. On Europa, two possibly new endemic species are being described by the CBNM. The first one, Achyranthes sp. nov., has been collected on Europa by Perrier de La Bâthie in 1921 but it has been reported erroneously as Centrostachys aquatica (R. Br.) Wall. (Cavaco, 1962). A further review of this woody and semi-lianescent shrub concludes that it doesn't refer to a known described taxon. This plant occurs in only one locality on Europa, in thickets of Pemphis acidula J.R. Forst. \& G. Forst. surrounding the mangrove. The second one, Euphorbia sp. nov., has been collected on Europa by Perrier de La Bâthie in 1921 too and it has been reported erroneously as Euphorbia glaucophylla Poir. (Denis, 1921). This very common herbaceous plant shows a large morphological variability and grows in all types of vegetation except mangroves and salt marshes, from the coast to the interior. Hypoestes juanensis (Acanthaceae) is described as strictly endemic to Juan de Nova (Perrier de La Bâthie, 1921). This species, very common on Juan de Nova, is typically a forest fringe plant showing a preference for semi-sciaphilous to semi-heliophylous edges, clearings and ventilated undergrowth. The species often forms a herbaceous layer, sometimes monospecific, included within different types of vegetation inside the island (not on the coastline) such as the vegetation dominated by the non-native naturalized Casuarina equisetifolia L. and other seminatural open vegetation, possibly resulting from recurrent and old fires. Another case concerns Boerhavia sp. nov.1, whose present characteristics include leaves with tangled margins and glandular hairs. It has been collected by Cremers (Battistini and Cremers, 1972) on the Iles Glorieuses and its originality has been raised by Boullet (2005). Reported to Juan de Nova in 2009, this species is common in the two territories where it occurs behind the coastline as a forest fringe. Another Boerhavia, sp. nov.2, is clearly distinct from the first one and seems to be strictly endemic of Tromelin where it is very common in the open. The last case concerns Perrierophytum glomeratum (Malvaceae) described in 2013 and considered as an exceptionally rare endemic species of Juan de Nova (Hivert et al., 2013). This species has been found in Juan de Nova in only three locations (5 individuals in total) in a Pisonia grandis R. Br. zone, a semi-natural Euphorbia stenoclada zone and a non-native Casuarina equisetifolia zone. The species was also recorded on Grande Glorieuse (Boullet, 2005; Hivert et al., 2014), where it is very abundant, occurring within natural and semi-natural vegetation units corresponding to an ancient dynamic dune system protected from direct oceanic influences, and to the limestone plateau formerly exploited by humans (Boullet, 2005). These taxa, therefore, cannot be considered as strictly endemic to Juan de Nova but rather as endemic to Juan de Nova and Grande Glorieuse.

\section{Biogeographical Remarks}

Although previously reported with a poor flora largely derived from South Madagascar's subarid coastal areas (Voeltzkow, 1904; Perrier De La Bâthie, 1921; Poisson, 1923; Decary, 1937; Bosser, 1952; Battistini, 1966; Capuron, 1966; Delépine et al., 1976; Le Corre and Jouventin, 1997; Caceres, 2003), Europa's native flora shows several remarkable features: the presence of possibly two strict endemic species (Achyranthes sp. nov. and Euphorbia sp. nov.); components of the halophilous flora of the salt marshes of the western Indian Ocean with five species (Salicornia pachystachya Bunge ex Ung.-Sternb., Suaeda monoica Forssk. ex J.F. Gmel., Tecticornia indica (Willd.) K.A. Sheph. \& Paul G. Wilson, Salsola littoralis, Sesuvium portulacastrum (L.) L.); the presence of seven species formerly considered as endemic to Madagascar (Cynanchum luteifluens, Dactyloctenium capitatum, Eragrostis capuronii, Euphorbia stenoclada, Ficus marmorata, Psiadia altissima, Salsola littoralis); and the presence of an endemic species of the western Indian Ocean (Panicum voeltzkowii Mez s. l.).

On Juan de Nova, the native flora is characterized by the presence of a strictly endemic species (Hypoestes juanensis), two endemic species restricted to the Mozambique channel, (Boerhavia sp. nov.1 and Perrierophytum glomeratum), 8 species formerly considered as endemic to Madagascar (Cheirolaena linearis, Cleome grandidieri, Dombeya greveana var. metameropsis, Euphorbia stenoclada, Maerua baillonii Hadj-Moust., Nesogenes madagascariensis, Psiadia altissima and Salvadora angustifolia), a species considered as endemic to Coral Seychelles (Aldabra) and Madagascar (Euphorbia mertonii Fosberg), as well as the presence of Panicum voeltzkowii Mez s. l. 
and Celosia spicata (Thouars) Spreng., two species endemic to the western Indian Ocean. The full taxonomic identification of distinct plant populations on Juan de Nova (Achyranthes spp. for example) could also reveal new interesting records.

The native flora of the Iles Glorieuses also presents some remarkable aspects. It is characterized by the presence of at least 13 regional endemic species: one endemic to the Mozambique channel cited in Juan de Nova and Grande Glorieuse (Perrierophytum glomeratum), one endemic to Iles Glorieuses and Juan de Nova (Boerhavia sp. nov.1), three species formerly considered as endemic to the Seychelles of the Aldabra group (Bulbostylis basalis, Euphorbia stoddartii, Portulaca mauritiensis var. aldabrensis), two species formerly considered as endemic to the Seychelles (Eragrostis subaequiglumis Renvoize and Nesogenes prostrata (Benth.) Hemsl.), one species formerly considered as endemic to Madagascar (Ficus grevei), and five species endemic to the western Indian Ocean (Adiantum hirsutum Bory, Celosia spicata, Disperis tripetaloides (Thouars) Lindl., Panicum voeltzkowii s. $l$. and Secamone pachystigma Jum. \& H. Perrier ). We can finally emphasize the presence of distinct populations whose study is in progress: Evolvulus alsinoides (L.) L., Ficus sp., Fimbristylis cymosa R. Br. s. l. and Solanum sp..

The main remarkable aspects of the native flora of Tromelin is the presence of a strictly endemic species (Boerhavia sp. nov.2) and of Portulaca mauritiensis var. aldabrensis, (ex-) endemic to the Aldabra group.

Note that the biogeographical status of restricted range taxa could evolve in the near future with dedicated prospecting on lesser known islands or countries in the vicinity of the Mozambique channel.

\section{Population Status, Distribution Range and Cultural Status of the Alien Flora}

Just like the native flora, regardless of the study area, the alien flora appears more diversified than stated in previous studies (Caceres, 2003). The alien flora of Europa (including many locally naturalized taxa with a relatively small area of occupancy on the island), is almost exclusively linked to anthropogenic habitats such as old and recent plantations, pathways, airstrips, and old fiber cultivation of Agave sisalana Perrine and Furcraea foetida (L.) Haw. Few alien plants have been cultivated on Europa (the majority of them for ornamental purposes) due to short time occupancy by humans and extreme conditions (a limiting factor for most introduced and cultivated species). Nevertheless, some alien plants are invasive in the area, such as Agave sisalana and Furcraea foetida found in Euphorbia stenoclada bushes, and Casuarina equisetifolia close to the coastlines. While the first two were formerly cultivated on a large scale in order to produce vegetal fibers, the reasons leading to the cultivation of $C$. equisetifolia on Europa remain less obvious (timber production, soil erosion, shade, wind breaks or water tanks).

The alien flora of Juan de Nova includes many naturalized taxa colonizing natural and semi-natural habitats, many of which are only cultivated on a small scale. These old practices reflect a tendency to artificialize the island and should be associated with Hector Patureau (manager of a guano and copra exploitation from 1952 to 1967) and other settlers. However, these plantations suffer mostly from the rough ecological conditions of this territory, notably those on sand during the dry season. Some native species of Juan de Nova have been cultivated as ornamentals (Dombeya greveana and Psiadia altissima) or for forestry exploitation (Cordia subcordata Lam., Salvadora angustifolia and Thespesia populneoides (Roxb.) Kostel.). To date, alien invasive (or potentially invasive) plants on Juan de Nova include Casuarina equisetifolia, Cocos nucifera L., Gossypium hirsutum L. and Lantana strigocamara R.W. Sanders.

The alien flora of the Iles Glorieuses has a very high proportion of naturalized taxa (mostly locally distributed within small areas) and a low proportion of cultivated taxa. These alien plants have largely been favored by past disturbance regimes (especially during the period of the coconut grove exploitation from 1885 to 1958) and current human activities. Therefore, alien plants can to date be found both in disturbed areas (residential areas, pathways, surroundings of the airstrip) and in natural or semi-natural habitats. Invasive alien plants on the Iles Glorieuses include Agave sisalana, Carica papaya L., Casuarina equisetifolia, Cocos nucifera, Flacourtia indica (Burm. f.) Merr. and Passiflora suberosa L..

The alien flora of Tromelin mostly includes non-naturalized taxa and cultivated taxa for ornamental and/or alimentary purposes, mostly close to the meteorological station (plants in poor health 
condition). This highlights a human will to modify this tiny island since the 1950's by introducing plants that can improve living conditions (ornamentals, shade) and human diet (Ananas comosus (L.) Merr., Capsicum frutescens L., Carica papaya, Citrus cf. sinensis (L.) Osbeck, Curcuma amada Roxb., Piper nigrum L. or even Solanum lycopersicum L.; taxa cultivated in pots, all excluded from analysis). Among the invasive aliens of Tromelin are included Cocos nucifera, Consolea falcata (Ekman \& Werderm.) F.M. Knuth, Euphorbia hirta L. and E. prostrata Aiton.

\section{CONCLUSION}

This study has improved knowledge about the vascular flora of the Iles Eparses, which was poorly known until recently, and our understanding of the flora of the western part of the Indian Ocean. In the case of native taxa, their density per $\mathrm{km}^{2}$ is far higher for the Iles Eparses than for the Aldabra group, given their small terrestrial surface area. The flora of each island is conditioned by the latitudinal gradient on the islands and by the climatic conditions specific to each territory. It is also conditioned by the proximity of nearby large source territories such as Madagascar, for the flora of Europa and Juan de Nova, and the Seychelles for the Iles Glorieuses. The rate of endemism observed on the Iles Eparses is very low, but will no doubt slightly increase as new species are described. Finally, with regard to threats to native flora by naturalized taxa, a management program for the eradication of Agave sisalana and Furcraeae foetida has been underway on Europa since 2011 in partnership with the TAAF and the FAZSOI while another program targeted against all (potential) invasive species has begun in 2016 on Tromelin in partnership with the TAAF.

\section{ACKNOWLEDGMENTS}

This study was made possible thanks to the financial and technical support of the Ministère de l'Écologie, du Développement Durable et de l'Énergie (MEDDE), of the Direction de l'Environnement, de l'Aménagement et du Logement de La Réunion (DEAL Réunion), of the CNRSINEE, of the Terres Australes et Antarctiques Françaises (TAAF), of the Forces Armées en Zone Sud de l'Océan Indien (FAZSOI) and the entire staff of the CBNM - especially Marie Lacoste, Christian Fontaine, Johnny Férard, Jérémy Beaurepaire, Benoit Dumeau, Betty Dufour, Antoine Chauvrat, Thibault Rochier, Frédéric Picot and Hervé Fossy - whom we heartily thank. Our thoughts also go to the dozens of scientists and military people we met during our expeditions. Finally, we also thank Frantz Hopkins and especially Brigitte Wiart-Boullet and John Thompson for their assistance in translation.

\section{REFERENCES}

Angiosperm Phylogeny Group (A.P.G.), 2009. An update of the Angiosperm Phylogeny Group classification for the orders and families of flowering plants: APG III. Botanical Journal of the Linnean Society 161, 105-121.

Barré, N., and J. Servan. 1988. L'avifaune des Iles éparses. In Thibault J.-C. et Guyot I., Eds. Livre rouge des oiseaux menaces des régions françaises d'Outre-Mer. Saint-Cloud, CIPO/ICBP Monographie 5, 209-224.

Battistini, R. 1966. La morphologie de l'île Europa. Mission scientifique à l'île Europa. L. R. Paris, Éditions du Muséum 91, 7-18.

Battistini, R., and G. Cremers. 1972. Geomorphology and vegetation of Iles Glorieuses. Atoll Research Bulletin 159, 1-25.

Bosser, J. 1952. Note sur la végétation des îles Europa et Juan de Nova. Le Naturaliste Malgache 4(1), 41-42.

Bosser, J., T. Cadet, J. Guého, H. R. Julien, and W. Marais. eds. 1976-2009. Flore des Mascareignes: La Réunion, Maurice, Rodrigues. 25 volumes parus. Mauritius Sugar Industry Research Institute (MSIRI), Port Louis, Maurice; Office de la Recherche Scientifique et Technique d'Outre-Mer 
(ORSTOM), Paris, France and Royal Botanic Garden, Kew, England, UK.

Boullet, V. 2005. Mission îles Glorieuses (10-16 Août 2005) - Flore et végétation. Pré-rapport non publié, Conservatoire Botanique National de Mascarin, île de La Réunion, 7 p. http://ileseparses.cbnm.org/index.php/presentation/actions-du-cbm?showall=\&start $=2$

Boullet, V. 2006. Mission île Europa (24 Mai-3 Juin 2006) - Flore et Végétation. Pré-rapport non publié, Conservatoire Botanique National de Mascarin, île de La Réunion, 11 p. http://ileseparses.cbnm.org/index.php/presentation/actions-du-cbm?showall=\&start $=2$

Boullet, V. 2008a. Typologie détaillée de la végétation et des habitats de l'île d'Europa. Fascicule 1: Systèmes de mangroves lagonnaires coralliennes. Rapport technique non publié, Conservatoire Botanique National de Mascarin, 12 p. http://ileseparses.cbnm.org/index.php/presentation/actionsdu-cbm?showall $=\&$ start $=2$

Boullet, V. 2008b. Typologie détaillée de la végétation et des habitats de l'île d'Europa - Fascicule 2: Systèmes de sansouires et des steppes salées coralliennes. Rapport technique non publié, Conservatoire Botanique National de Mascarin, 38 p. http://ileseparses.cbnm.org/index.php/presentation/actions-du-cbm?showall=\&start $=2$

Boullet, V. 2014. La végétation de l'île Europa (canal du Mozambique). 1 - Sansouïres et steppes salées. Documents phytosociologiques, série 3, 1, 500-535.

Bourjea, J., S. Ciccione, M. Lauret-Stepler, C. Marmoex, and C. Jean. 2011. Les îles Eparses, vingtcinq ans de recherche sur les tortues marines. Bull. la Société Herpétologique Fr. 140, 95-111.

Brummit, R. K., and C. E. Powell. 1992. Authors of plant names. Royal Botanic Gardens, Kew, 732 p.

Caceres, S. 2003. Étude préalable pour le classement en réserve Naturelle des Îles Éparses. Mémoire de DESS Sciences et gestion de l'environnement tropical de l'Université de la Réunion, $147 \mathrm{p}$. http://poupin.joseph.free.fr/pdf/caceres-2003-iles-eparses-classement.pdf

Cadet, T. 1984. Mission aux Glorieuses, 28-29 décembre 1984. Saint Denis, La Réunion, Université de La Réunion, 8 p.

Camoin, G. F., M. Colonna, L. F. Montaggioni, J. Casanova, G. Faure, and B.A. Thomassin. 1997. Holocene sea level changes and reef development in southwestern Indian Ocean. Coral Reefs 16 : 247-259.

Camoin, G. F., L. F. Montaggioni, and C. J. R. Braithwaite. 1984. Late glacial to post glacial sea levels in the Western Indian Ocean. Marine Ecology 206 : 119-146.

Capuron, R. 1966. Rapport succinct sur la végétation et la flore de l'île Europa. Mission scientifique à l'île Europa. R. Legendre Paris, Éditions du Muséum 91, 19-22.

Carlton, J. T. 1996. Biological Invasions and Cryptogenic Species. Ecology 77(6), 1653-1655.

Chabanet, P., and P. Durville. 2005. Reef fish inventory of Juan de Nova's natural park (Western Indian Ocean). Western Indian Ocean Journal of Marine Science 4(2), 145-162.

Chabanet, P., L. Bigot, J.-B. Nicet, P. Durville, L. Massé, T. Mulochau, C. Russo, E. Tessier, and D. Obura. 2016. Coral reef monitoring in the iles eparses, Mozambique channel (2011-2013). Acta oecologica 72 (2016) 62-71.

Cointat, C. 2010. Les îles Éparses, terres d'avenir. Rapport d'information n² 299, Sénat session ordinaire de 2009-2010, Commission des lois constitutionnelles, de législation, du suffrage universel, du Règlement et d'administration générale et du groupe d'études sur l'Arctique, l'Antarctique et les Terres australes, sur les îles Éparses. Espace Librairie du Sénat.

Conand, C., T. Mulochau, S. Stöhr, M. Eléaume, and P. Chabanet. 2016. Inventory of Echinoderms in the Iles Eparses (Europa, Glorieuses, Juan de Nova), Mozambique Channel, France. Acta oecologica 72 (2016) 53-61.

Conservatoire Botanique National de Mascarin (Gigord L. D. B. coordinateur, Hivert J. \& Cuidet Y. auteurs principaux) 2013a. - Flore et végétations des îles Éparses. http://ileseparses.cbnm.org/

Conservatoire Botanique National de Mascarin (Boullet V. coord.), 2013b. Index de la flore vasculaire de Mayotte (Trachéophytes): statuts, menaces et protections. Version tableur 2013.2 (mise à jour du 26 Juin 2013). Conservatoire Botanique National de Mascarin, Saint-Leu (Réunion).

Conservatoire Botanique National de Mascarin (Boullet V. coord.), 2017. Index de la flore vasculaire de la Réunion (Trachéophytes): statuts, menaces et protections. Version tableur 2015.1 (mise à jour du 19 Janvier 2017). Conservatoire Botanique National de Mascarin, Saint-Leu (Réunion). 
Decary, R. 1937. Les satellites de Madagascar et l'ancienne navigation dans le Canal du Mozambique. Bulletin de l'Académie, Madagascar, 20, 53-72.

Delépine, R., L. A. Maugé, and A. Padovani. 1976. Observations écologiques et climatologiques dans les îles Europa, Glorieuses et Tromelin. Biologie marine et exploitation des ressources de l'océan Indien occidental. Saint Denis, La Réunion, ORSTOM.

Dumeau, B., J. Hivert, and L. D. B. Gigord. 2014. L'avifaune de l'île d'Europa, la plus grande des îles Éparses, entre Afrique et Madagascar. Ornithos 21(1), 14-37.

Durville, P., P. Chabanet, and J.-P. Quod. 2003. Visual Census of the Reef Fishes in the Natural Reserve of the Glorieuses Islands (Western Indian Ocean). Western Indian Ocean Journal of Marine Science 2(2), 95-104.

Ellenberg, H., and D. Mueller-Dombois. 1967. A key to Raunkiaer plant life forms with revised subdivisions. Ber. geobot. Inst. E.T.H., Stiftg Rübel, Zurich, 37: 56-73.

Fosberg, F. R. 1956. Military geography of the northern Marshall Islands. Intelligence Division, Office of the Engineer, Headquarters U.S.A.F. (Far East), Tokyo, XI + 320 p.

Fosberg, F. R. 1971. Preliminary survey of Aldabra vegetation. Philosophical transactions of the Royal Society of London, 260: 215-225.

Fosberg, F. R., and S. A. Renvoize. 1980. The flora of Aldabra and neighbouring islands. Kew Bulletin Additional Series VII, 358 p.

Frain, I. 2009. Les naufragés de Tromelin. Éditions Michel Lafon, 343 p.

Friedmann, F. 1994. Flore des Seychelles - Dicotylédones. Collection Didactiques, Éditions de l'Orstom, Paris, $663 \mathrm{p}$.

Friedmann, F. 2011. Flore des Seychelles - Dicotylédones. Collection Faune et flore tropicales , IRD Éditions/MNHN, $663 \mathrm{p}$.

Gibson, C. W. D., and J. Phillipson. 1983. The vegetation of Aldabra Atoll: preliminary analysis and explanation of the vegetation map. Philosophical Transactions of the Royal Society of London, 302: 201-235.

Goldberg, W. M. 2016. Atolls of the world: revisiting the original checklist. Atoll Research Bulletin, 610: 1-47.

Gravier-Bonnet, N., and C. Bourmaud. 2006a. Hydroids (Cnidaria, Hydrozoa) of coral reefs: preliminary results on community structure, species distribution and reproductive biology in Juan de Nova island (Southwest Indian Ocean). Western Indian Ocean Journal of Marine Science 5(2), 123-132.

Gravier-Bonnet, N., and C. Bourmaud. 2006b. Hydroids (Cnidaria, Hydrozoa) of coral reefs: preliminary results on community structure, species distribution and reproductive biology in the Îles Glorieuses (Southwest Indian Ocean). 10th Int. Coral Reef Symposium, Okinawa, Japon, pp. 188-196.

Hivert, J., B. Dufour, and L. D. B., Gigord. 2014. Compte-rendu scientifique et technique de mission de longue durée d'étude de la flore et des habitats des Glorieuses (Janvier-Mars 2014). Rapport non publié, Conservatoire Botanique National de Mascarin, île de La Réunion, 60 p. http://ileseparses.cbnm.org/index.php/presentation/actions-du-cbm?showall=\&start $=2$

Hivert, J., J. Férard, C. Fontaine, and L. D. B. Gigord. 2013. Compte-rendu scientifique et technique de mission de longue durée d'étude de la flore et des habitats de Juan de Nova (Mars 2013). Rapport non publié, Conservatoire Botanique National de Mascarin, île de La Réunion, 70 p. http://ileseparses.cbnm.org/index.php/presentation/actions-du-cbm?showall=\&start=2

Hnatuik, S. H. 1979. Numbers of plant species on the islands of Aldabra Atoll. Philosophical Transactions of the Royal Society of London, 286: 247-254.

Hoareau, A. 1993. Les îles Éparses: histoire et découverte. Azalées éditions, Saint-André, La Réunion, $239 \mathrm{p}$.

Humbert, H., and J.-F. Leroy. 1936-2001. Flore de Madagascar et des Comores: plantes vasculaires. Publiée sous les auspices du gouvernement général de Madagascar et du Muséum National d'Histoire Naturelle de Paris, Tananarive et Paris.

Larrue, S., J.-F. Butaud, P. Dumas, and S. Ballet. 2015. Native plant species richness on Eastern Polynesia's remote atolls: Which abiotic factors influence its spatial pattern? Progress in Physical Geography, Vol. 40(1), 112-134. 
Le Corre, M., and P. Jouventin. 1997. Ecological significance and conservation priorities of Europa Island (western Indian Ocean), with special reference to seabirds. Terre et Vie (Revue d'écologie) 52, 205-220.

Le Corre, M., and J.-M. Probst. 1997. Migrant and vagrant birds of Europa Island (southern Mozambique Channel). Ostrich 68, 13-18.

Le Corre, M., and R. J. Safford. 2001. La Réunion and Iles Éparses. Pp 693-702 in Fishpool, L.D.C. and Evans M.I. (eds) Important Bird Areas of Africa and associated islands. Cambridge, UK: Bird Life International.

Le Gall, J.-Y., A. Lebeau, and J. Kopp. 1985. Évaluation de la production de tortues vertes Chelonia mydas nouveau-nés sur les sites de ponte Europa et Tromelin (Océan Indien). Océanogr. Trop., 20 (2), 117-133.

Madagascar Catalogue. 2017. Catalogue of the Vascular Plants of Madagascar. Missouri Botanical Garden, St. Louis, U.S.A. \& Antananarivo, Madagascar. http://www.efloras.org/madagascar

Météo-France. 2016. Données climatiques mensuelles sur les îles Éparses (Europa, Juan de Nova, Les Glorieuses, Tromelin) sur la période 1951-2016.

Mitić, B., I. Boršić, I. Dujmović, S. Bogdanović, M. Milović, P. Cigić, I. Rešetnik, and T. Nikolić. 2008. Alien flora of Croatia: proposals for standards in terminology, criteria and related database. Nat. Croat., 17(2), 73-90.

Mueller-Dombois, D., and R. F. Fosberg. 1998. Vegetation of the Tropical Pacific Islands. SpringerVerlag, New York, Ecological Studies, vol. 132, 733 p., 521 illustr.

Ocean Surface Current Analyses, Real Time (OSCAR). 2015. National Oceanic and Atmospheric Administration. http://www.oscar.noaa.gov/datadisplay/oscar latlon.php

Paulian, R. 1955. Observations sur la faune terrestre de l'île Tromelin. Le Naturaliste Malgache 4(1), 41-42.

Perrier De La Bâthie, H. 1921. Note sur la constitution géologique et la flore des îles Chesterfield, Juan de Nova, Europa et Nosy-Trozona. Bulletin économique de Madagascar 1921, 170-176.

Pyšek, P., D. M. Richardson, M. Rejmánek, G. L. Webster, M. Williamson, M., and J. Kirschner. 2004. Alien plants in checklists and floras: towards better communication between taxonomists and ecologists. Taxon 53(1), 131-143.

Poisson, H. 1923. Rapport de tournée à l'île Europa, Morombe et retour par terre, via Manombo (du 16 au 26 février 1923). Bulletin économique de Madagascar, 131-141.

Polhill, R. M. (ed.) 1948-2012. Flora of Tropical East Africa. Env. 200 vol. parus. A. A. Balkema, Rotterdam, Royal Botanic Gardens, Kew.

Pope, G. V., et al. (ed.) 1960-(2005). Flora Zambesiaca: Mozambique, Malawi, Zambia, Rhodesia, Botswana. 32 vol. parus. Flora Zambesica Managing Committee.

Poupin, J. 2016. First inventory of the Crustacea (Decapoda, Stomatopoda) of Juan de Nova Island with ecological observations and comparison with nearby islands in the Mozambique channel (Europa, Glorieuses, Mayotte). Acta oecologica 72 (2016) 41-52. http://dx.doi.org/10.1016/j.actao.2015.04.001

Poupin, J., M. Zubia, N. Gravier-Bonnet, P. Chabanet, and A. Duhec. 2013a. Crustacea Decapoda of Glorieuses Islands, with notes on the distribution of the coconut crab (Birgus latro) in the western Indian Ocean. Marine Biodiversity Records, 1-12.

Poupin, J., M. Zubia, N. Gravier-Bonnet, P. Chabanet, and M. Malay. 2013b. Illustrated Checklist of the Decapoda at Europa Island. Western Indian Ocean Journal of Marine Science 11(1), 1-25.

Probst, J.-M, R. Tézier, P. Houchois, G. Sourice, L. Reynaud, C. Villedieu, M. Banderier, P. Barroil, S. Ciccione, H. Sauvignet, D. Roos, and G. Bertrand. 2000. Inventaire des Oiseaux, des Reptiles et des Mammifères de l'Archipel des Glorieuses (îles éparses de l'Océan Indien). Bulletin Phaethon 11, 31-50.

Probst, J.-M, R. Tézier, P. Barroil, G. Bertrand, N. Villeneuve, F. Moullin, and J. Lehir. 2001. Compte-rendu d'observation des vertébrés terrestres d'Europa. Mission du 10 novembre au 16 Décembre 2001 (Canal du Mozambique). Bulletin Phaethon 14, 92-99.

Pryer, K.M., E. Schuettpelz, P. G. Wolf, H. Schneider, A. R. Smith, and R. Cranfill. 2004. Phylogeny and evolution of ferns (Monilophytes) with a focus on the early leptosporangiate divergences. American Journal of Botany 91(10), 1582-1598. 
Quétel, C., S. Marinesque, D. Ringler, L. Fillinger, T. Changeux, C. Marteau, and M. Trousselier. 2016. Iles Eparses (SW Indian Ocean) as reference ecosystems for environmental research. Acta oecologica (2016). http://dx.doi.org/10.1016/j.actao.2015.12.012

Quod, J. P., A. Barrere, P. Chabanet, P. Durville, J.-B. Nicet, and R. Garnier. 2007. The status of the coral reefs of French scattered islands in the Indian Ocean. Revue d'écologie - La Terre et la Vie, 2007, 62 (1), 3-16.

Richardson, D. M., P. Pyšek, M. Rejmánek, M. G. Barbour, F. D. Panetta, and C. J. West. 2000. Naturalization and invasion of alien plants: concepts and definitions. Diversity and Distributions, 6, 93-107. Speke, J. H. 1863. Journal of the Discovery of the Source of the Nile. William Blackwood and Sons, Edinburgh and London, MDCCCLXIII, 658 p.

Staub, F. 1970. Geography and ecology of Tromelin Island. Atoll Research Bulletin 136, 197-209.

Stevens, P. F., 2001-2015. Angiosperm Phylogeny Website. Version 14, April 2015. http://www.mobot.org/mobot/research/apweb/

Stoddart, D. R. 1992. Biogeography of the Tropical Pacific. Pacific Science 46 (2), 276-293.

Terres Australes et Antarctiques Françaises. 2011. Les îles éparses: Europa, Bassas da India, Juan de Nova, Glorieuses, Tromelin. Livret de découverte des îles Éparses, TAAF, 28 p. http://www.taaf.fr/IMG/pdf/livret eparses_2016web.pdf

Terres Australes et Antarctiques Françaises, 2016. http://www.taaf.fr/The-French-Southern-andAntarctic-Lands

Thaman, R. R. 2016. The Flora of Tuvalu: Lakau Mo Mouku o Tuvalu. Atoll Research Bulletin 611, 1129.

Thaman, R. R., and M. Samuelu. 2016. Vascular plants, vegetation and ethnobotany of Banaba (ocean island), Republic of Kiribati. Atoll Research Bulletin 609, 1-91.

The International Plant Names Index, 2017. http://www.ipni.org/index.html

Thulin, M. (ed.) 1993-2006. Flora of Somalia. 4 volumes. Royal Botanics Gardens, Kew.

USDA, ARS, National Genetic Resources Program. 2017. Germplasm Resources Information Network - (GRIN) [National Plant Germplasm System]. National Germplasm Resources Laboratory, Beltsville, Maryland. Available at https://npgsweb.arsgrin.gov/gringlobal/taxonomybrowse.aspx (accessed October 2015).

Virah-Sawmy, M., K. J. Willis, and L. Gillson. 2009. Threshold response of Madagascar's littoral forest to sea-level rise. Global Ecology and Biogeography (Global Ecol. Biogeogr.) 18 : 98-110.

Voeltzkow, A. 1904. Berichte uber eine Reise nach Ost Africa zur Untersuchung der Bildung und des Aufbaues der Riffe und inseln des westlichen Indischen Ozeans. Berichte I und II s. diese Zeitschrift., pp. 27.

Woodroffe, S. A., and B. J. Horton. 2005. Holocene sea-level changes in the Indo-Pacific. Journal of Asian earth sciences 25 (1): 29-43. 


\section{APPENDIX A}

Table 2. List of all taxa found on the Iles Eparses. In the column 'Life forms' differents codes proposed by Ellenberg and Mueller-Dombois (1967) were used and applied to level 3: P scap = trees; P caesp = shrubs; P ros = tuft trees; P dol = bottle trees; P succ = tall succulents; $\mathrm{P}$ gram $=$ phanerophytic grasses; $\mathrm{P}$ herb = phanerophytic forbs; Ch frut caesp = caespitose woody dwarf-shrubs; Ch frut rept $=$ reptant woody dwarf-shrubs; Ch suff caesp = caespitose semi-woody dwarfshrubs; Ch suff rept = reptant semi-woody dwarf-shrubs; Ch suff scap = scapose semi-woody dwarf-shrubs; Ch herb caesp = caespitose herbaceous

chamaephytes; Ch herb rept $=$ reptant herbaceous chamaephytes; $\mathrm{Ch}$ herb pulv = pulvinate herbaceous chamaephytes; $\mathrm{Ch}$ st succ $=$ low succulents with stemsucculents; Ch 1 succ = low succulents with leaf-succulents; $\mathrm{EL}=$ hemi-epiphytes, pseudo-lianas , epiphytic lianas; $\mathrm{H}$ caesp = caespitose hemicryptophytes; $\mathrm{H}$ rept $=$ reptant hemicryptophytes; $\mathrm{H}$ scap = scapose hemicryptophytes without rosette; $\mathrm{c} \mathrm{G}$ bulb = rain-green bulbous geophytes; $\mathrm{c} \mathrm{G}$ rhiz $=$ rain-green rhizomegeophytes; $\mathrm{T}$ caesp $=$ caespitose therophytes; $\mathrm{T}$ rept $=$ reptant therophytes; $\mathrm{T}$ scap $=$ therophytes without rosette; $\mathrm{T}$ ros $=$ therophytes with rosette; $\mathrm{T}$ sem $=$ therophytes with semi-rosette; $\mathrm{T}$ succ $=$ succulent therophytes; st $\mathrm{PL}=$ phanerophytic and chamaephytic winding climbers; el PL = phanerophytic and chamaephytic tendril climbers; d PL = phanerophytic and chamaephytic spread-climbers; st HL = hemicryptophytic winding climbers; el HL = hemicryptophytic tendril climbers; el GL = geophytic tendril climbers; $\mathrm{d}$ TL = throphytic spread climbers. In the column 'Voucher herbarium specimens' differents acronyms were used to precise the name of the herbarium were herbarium specimens have been deposited : $\mathrm{CBNM}=\mathrm{CBNM}$ herbarium ; $\mathrm{MO}=\mathrm{Missouri}$ Botanical Garden herbarium ; $\mathrm{P}=$ Muséum National d'Histoire Naturelle de Paris herbarium

\begin{tabular}{|c|c|c|c|c|c|c|c|c|c|}
\hline \multirow[t]{2}{*}{ Scientific name } & \multirow[t]{2}{*}{ Family } & \multirow{2}{*}{$\begin{array}{l}\text { Life } \\
\text { forms }\end{array}$} & \multirow{2}{*}{$\begin{array}{l}\text { General status and } \\
\text { distribution range }\end{array}$} & \multicolumn{4}{|c|}{ Presence } & \multirow{2}{*}{$\begin{array}{l}\text { First record } \\
\text { source from } \\
\text { (name of the } \\
\text { territory) }\end{array}$} & \multirow{2}{*}{$\begin{array}{l}\text { Voucher herbarium } \\
\text { specimens (name of the } \\
\text { territory and acronym of } \\
\text { the herbarium) }\end{array}$} \\
\hline & & & & $\begin{array}{l}\text { Europ } \\
\quad a \\
\text { (EUR) }\end{array}$ & $\begin{array}{l}\text { Juan } \\
\text { de } \\
\text { Nova } \\
\text { (JDN) }\end{array}$ & $\begin{array}{l}\text { lles } \\
\text { Glorieuses } \\
\text { (GLO) }\end{array}$ & $\begin{array}{l}\text { Tromelin } \\
\text { (TRO) }\end{array}$ & & \\
\hline $\begin{array}{l}\text { Abutilon } \\
\text { pseudocleistogamum } \\
\text { Hochr. }\end{array}$ & Malvaceae & $\begin{array}{l}\text { Ch frut } \\
\text { caesp }\end{array}$ & Madagascar & $x$ & & & & $\begin{array}{l}\text { Bibliography } \\
\text { (EUR) }\end{array}$ & EUR: CBNM, MO, P \\
\hline Acalypha indica L. & Euphorbiaceae & T scap & $\begin{array}{l}\text { Supposed native from Asia } \\
\text { and tropical Africa }\end{array}$ & & $x$ & $x$ & & $\begin{array}{l}\text { Bibliography } \\
\text { (GLO); Field } \\
\text { work (JDN) }\end{array}$ & $\begin{array}{l}\text { JDN: CBNM, MO, P; GLO: } \\
\text { CBNM, MO, P }\end{array}$ \\
\hline Acalypha indica L. & Cactaceae & P succ & $\begin{array}{l}\text { Central America, from South } \\
\text { of United States of America } \\
\text { (Florida, Texas) to the } \\
\text { Caribbean and Venezuela }\end{array}$ & & $x$ & & & $\begin{array}{l}\text { Field work } \\
\text { (JDN) }\end{array}$ & \\
\hline
\end{tabular}




\begin{tabular}{|c|c|c|c|c|c|c|c|c|c|}
\hline \multirow[t]{2}{*}{ Scientific name } & \multirow[t]{2}{*}{ Family } & \multirow{2}{*}{$\begin{array}{l}\text { Life } \\
\text { forms }\end{array}$} & \multirow{2}{*}{$\begin{array}{l}\text { General status and } \\
\text { distribution range }\end{array}$} & \multicolumn{4}{|c|}{ Presence } & \multirow{2}{*}{$\begin{array}{l}\text { First record } \\
\text { source from } \\
\text { (name of the } \\
\text { territory) }\end{array}$} & \multirow{2}{*}{$\begin{array}{l}\text { Voucher herbarium } \\
\text { specimens (name of the } \\
\text { territory and acronym of } \\
\text { the herbarium) }\end{array}$} \\
\hline & & & & $\begin{array}{l}\text { Europ } \\
\quad a \\
\text { (EUR) }\end{array}$ & $\begin{array}{l}\text { Juan } \\
\text { de } \\
\text { Nova } \\
\text { (JDN) }\end{array}$ & $\begin{array}{c}\text { Iles } \\
\text { Glorieuses } \\
\text { (GLO) }\end{array}$ & $\begin{array}{l}\text { Tromelin } \\
\text { (TRO) }\end{array}$ & & \\
\hline $\begin{array}{l}\text { Achyranthes aspera } \\
\text { L. var. aspera }\end{array}$ & Amaranthaceae & $\begin{array}{l}\text { Ch suff } \\
\text { scap }\end{array}$ & $\begin{array}{l}\text { Native from tropical Asia, } \\
\text { became almost pantropical }\end{array}$ & $x$ & $x$ & & & $\begin{array}{l}\text { Bibliography } \\
\text { (EUR); Field } \\
\text { work (JDN) }\end{array}$ & $\begin{array}{l}\text { EUR: CBNM, MO, P; JDN: } \\
\text { CBNM, MO, P }\end{array}$ \\
\hline $\begin{array}{l}\text { Achyranthes aspera } \\
\text { L. var. fruticosa } \\
\text { (Lam.) Boerl. }\end{array}$ & Amaranthaceae & $\begin{array}{l}\text { Ch suff } \\
\text { caesp / } \\
\text { d PL }\end{array}$ & Pantropical & & $x$ & $x$ & & $\begin{array}{l}\text { Bibliography } \\
\text { (JDN, GLO) }\end{array}$ & $\begin{array}{l}\text { JDN: CBNM, MO; GLO: } \\
\text { CBNM, MO, P }\end{array}$ \\
\hline $\begin{array}{l}\text { Achyranthes aspera } \\
\text { L. var.1 }\end{array}$ & Amaranthaceae & $\begin{array}{l}\text { Ch suff } \\
\text { caesp }\end{array}$ & ? & & $x$ & & & $\begin{array}{l}\text { Field work } \\
\text { (JDN) }\end{array}$ & JDN: CBNM, MO, P \\
\hline $\begin{array}{l}\text { Achyranthes aspera } \\
\text { L. var. } 2\end{array}$ & Amaranthaceae & $\begin{array}{l}\text { Ch suff } \\
\text { caesp }\end{array}$ & $?$ & & $x$ & & & $\begin{array}{l}\text { Field work } \\
\text { (JDN) }\end{array}$ & JDN: CBNM, MO, P \\
\hline $\begin{array}{l}\text { Achyranthes sp.nov. } \\
?\end{array}$ & Amaranthaceae & P caesp & Europa (endemic ?) & $x$ & & & & $\begin{array}{l}\text { Bibliography } \\
\text { (EUR) }\end{array}$ & EUR: CBNM, MO, P \\
\hline $\begin{array}{l}\text { Achyranthes cf. } \\
\text { talbotii Hutch. \& } \\
\text { Dalziel }\end{array}$ & Amaranthaceae & $\begin{array}{l}\text { Ch suff } \\
\text { caesp }\end{array}$ & Madagascar, West Africa & $x$ & & & & $\begin{array}{l}\text { Bibliography } \\
\text { (EUR) }\end{array}$ & EUR: CBNM, MO, P \\
\hline $\begin{array}{l}\text { Acrostichum aureum } \\
\text { L. }\end{array}$ & Pteridaceae & $\begin{array}{l}\text { Ch herb } \\
\text { caesp / } \\
\text { P herb }\end{array}$ & Pantropical & & $x$ & & & $\begin{array}{l}\text { Field work } \\
\text { (JDN) }\end{array}$ & JDN: CBNM, MO, P \\
\hline Adansonia digitata $\mathrm{L}$. & Malvaceae & P dol & $\begin{array}{l}\text { Tropical Africa, mostly } \\
\text { ornamental (sub-humid and } \\
\text { semi-arid regions in the South } \\
\text { of the Sahara), Comoros, } \\
\text { South Saudi Arabia; probably } \\
\text { introduced and naturalized in } \\
\text { Madagascar; introduced and } \\
\text { cultivated elsewhere in } \\
\text { tropical regions }\end{array}$ & & $x$ & & & $\begin{array}{l}\text { Bibliography } \\
\text { (JDN) }\end{array}$ & \\
\hline
\end{tabular}




\begin{tabular}{|c|c|c|c|c|c|c|c|c|c|}
\hline \multirow[t]{2}{*}{ Scientific name } & \multirow[t]{2}{*}{ Family } & \multirow{2}{*}{$\begin{array}{l}\text { Life } \\
\text { forms }\end{array}$} & \multirow{2}{*}{$\begin{array}{l}\text { General status and } \\
\text { distribution range }\end{array}$} & \multicolumn{4}{|c|}{ Presence } & \multirow{2}{*}{$\begin{array}{l}\text { First record } \\
\text { source from } \\
\text { (name of the } \\
\text { territory) }\end{array}$} & \multirow{2}{*}{$\begin{array}{l}\text { Voucher herbarium } \\
\text { specimens (name of the } \\
\text { territory and acronym of } \\
\text { the herbarium) }\end{array}$} \\
\hline & & & & $\begin{array}{c}\text { Europ } \\
a \\
\text { (EUR) }\end{array}$ & $\begin{array}{l}\text { Juan } \\
\text { de } \\
\text { Nova } \\
\text { (JDN) }\end{array}$ & $\begin{array}{c}\text { lles } \\
\text { Glorieuses } \\
\text { (GLO) }\end{array}$ & $\begin{array}{l}\text { Tromelin } \\
\text { (TRO) }\end{array}$ & & \\
\hline $\begin{array}{l}\text { Adiantum hirsutum } \\
\text { Bory }\end{array}$ & Pteridaceae & $\begin{array}{l}\text { Ch herb } \\
\text { caesp / } \\
\text { Ch herb } \\
\text { rept }\end{array}$ & $\begin{array}{l}\text { Madagascar, Mascarene } \\
\text { islands (Reunion and } \\
\text { Mauritius) }\end{array}$ & & & $x$ & & $\begin{array}{l}\text { Field work } \\
\text { (GLO) }\end{array}$ & GLO: CBNM, MO, P \\
\hline $\begin{array}{l}\text { Adiantum } \\
\text { philippense L. }\end{array}$ & Pteridaceae & $\begin{array}{l}\text { Ch herb } \\
\text { caesp }\end{array}$ & $\begin{array}{l}\text { Pantropical and } \\
\text { pansubstropical }\end{array}$ & & & $x$ & & $\begin{array}{l}\text { Field work } \\
\text { (GLO) }\end{array}$ & GLO: CBNM, MO \\
\hline $\begin{array}{l}\text { Agave sisalana } \\
\text { Perrine }\end{array}$ & Asparagaceae & $\begin{array}{l}\text { Ch I } \\
\text { succ }\end{array}$ & $\begin{array}{l}\text { Eastern Mexico; mostly } \\
\text { cultivated elsewhere }\end{array}$ & $x$ & $x$ & $x$ & & $\begin{array}{l}\text { Bibliography } \\
\text { (EUR); Field } \\
\text { work (JDN, } \\
\text { GLO) }\end{array}$ & GLO: CBNM \\
\hline $\begin{array}{l}\text { Ageratum conyzoides } \\
\text { L. }\end{array}$ & Asteraceae & T caesp & Central and South America & & & $x$ & & $\begin{array}{l}\text { Field work } \\
\text { (GLO) }\end{array}$ & GLO: CBNM, MO, P \\
\hline $\begin{array}{l}\text { Albizia lebbeck (L.) } \\
\text { Benth. }\end{array}$ & Fabaceae & P scap & $\begin{array}{l}\text { Probably native from tropical } \\
\text { Asia; became pantropical }\end{array}$ & & $x$ & & & $\begin{array}{l}\text { Field work } \\
\text { (JDN) }\end{array}$ & \\
\hline $\begin{array}{l}\text { Alocasia } \\
\text { macrorrhizos (L.) G. } \\
\text { Don var. } \\
\text { macrorrhizos }\end{array}$ & Araceae & $\begin{array}{l}\text { Ch herb } \\
\text { caesp }\end{array}$ & $\begin{array}{l}\text { Sri Lanka, India to Malaysia, } \\
\text { introduced and cultivated in } \\
\text { many areas }\end{array}$ & & & $x$ & & $\begin{array}{l}\text { Field work } \\
\text { (GLO) }\end{array}$ & GLO: CBNM, MO, P \\
\hline Aloe vera (L.) Burm. $\mathrm{f}$. & $\begin{array}{l}\text { Xanthorrhoeace } \\
\text { ae }\end{array}$ & $\begin{array}{l}\mathrm{Ch} \mathrm{I} \\
\text { succ }\end{array}$ & $\begin{array}{l}\text { South West Saudi Arabia } \\
\text { (often wrongly given as native } \\
\text { to the Canary islands); } \\
\text { cultivated as ornemental and } \\
\text { medicinal plant from very long } \\
\text { time and introduced from } \\
\text { very long time in } \\
\text { Mediterranean, Central and } \\
\text { South America }\end{array}$ & & & & $x$ & $\begin{array}{l}\text { Field work } \\
\text { (TRO) }\end{array}$ & \\
\hline
\end{tabular}




\begin{tabular}{|c|c|c|c|c|c|c|c|c|c|}
\hline \multirow[t]{2}{*}{ Scientific name } & \multirow[t]{2}{*}{ Family } & \multirow{2}{*}{$\begin{array}{l}\text { Life } \\
\text { forms }\end{array}$} & \multirow{2}{*}{$\begin{array}{l}\text { General status and } \\
\text { distribution range }\end{array}$} & \multicolumn{4}{|c|}{ Presence } & \multirow{2}{*}{$\begin{array}{l}\text { First record } \\
\text { source from } \\
\text { (name of the } \\
\text { territory) }\end{array}$} & \multirow{2}{*}{$\begin{array}{l}\text { Voucher herbarium } \\
\text { specimens (name of the } \\
\text { territory and acronym o } \\
\text { the herbarium) }\end{array}$} \\
\hline & & & & $\begin{array}{l}\text { Europ } \\
\text { a } \\
\text { (EUR) }\end{array}$ & $\begin{array}{l}\text { Juan } \\
\text { de } \\
\text { Nova } \\
\text { (JDN) }\end{array}$ & $\begin{array}{l}\text { Iles } \\
\text { Glorieuses } \\
\text { (GLO) }\end{array}$ & $\begin{array}{l}\text { Tromelin } \\
\text { (TRO) }\end{array}$ & & \\
\hline $\begin{array}{l}\text { Alternanthera } \\
\text { pungens Kunth }\end{array}$ & Amaranthaceae & $\begin{array}{l}\text { Ch herb } \\
\text { rept }\end{array}$ & $\begin{array}{l}\text { South America, became } \\
\text { pantropical }\end{array}$ & $x$ & & & & $\begin{array}{l}\text { Bibliography } \\
\text { (EUR) }\end{array}$ & EUR: CBNM, MO, P \\
\hline $\begin{array}{l}\text { Amaranthus } \\
\text { graecizans L. subsp. } \\
\text { silvestris (Vill.) } \\
\text { Brenan }\end{array}$ & Amaranthaceae & T caesp & Cosmopolitan & $x$ & $x$ & & & $\begin{array}{l}\text { Field work } \\
\text { (EUR, JDN) }\end{array}$ & $\begin{array}{l}\text { EUR: CBNM, MO, P; JDN: } \\
\text { CBNM, MO, P }\end{array}$ \\
\hline $\begin{array}{l}\text { Amaranthus spinosus } \\
\text { L. }\end{array}$ & Amaranthaceae & T caesp & Pantropical & & & $x$ & & $\begin{array}{l}\text { Field work } \\
\text { (GLO) }\end{array}$ & GLO: CBNM \\
\hline Amaranthus viridis L. & Amaranthaceae & T caesp & $\begin{array}{l}\text { Obscure origin (supposed } \\
\text { native from South America), } \\
\text { became } \pm \text { pantropical; } \\
\text { naturalized in warm and } \\
\text { temperate regions }\end{array}$ & $x$ & $x$ & $x$ & & $\begin{array}{l}\text { Bibliography } \\
\text { (EUR); Field } \\
\text { work (JDN, } \\
\text { GLO) }\end{array}$ & EUR: CBNM, MO, $\mathrm{P}$ \\
\hline Annona muricata $\mathrm{L}$. & Annonaceae & P caesp & $\begin{array}{l}\text { Tropical Central America ; } \\
\text { widely cultivated in tropical } \\
\text { regions }\end{array}$ & & & $x$ & & $\begin{array}{l}\text { Field work } \\
\text { (GLO) }\end{array}$ & GLO: CBNM, MO, P \\
\hline Annona squamosa $\mathrm{L}$. & Annonaceae & P caesp & $\begin{array}{l}\text { Supposed native from Antillas } \\
\text {; widely cultivated in tropical } \\
\text { regions }\end{array}$ & & & $x$ & & $\begin{array}{l}\text { Field work } \\
\text { (GLO) }\end{array}$ & GLO: CBNM, MO, P \\
\hline $\begin{array}{l}\text { Antigonon leptopus } \\
\text { Hook. \& Arn. }\end{array}$ & Polygonaceae & el PL & $\begin{array}{l}\text { Mexico and Central America } \\
\text { (Guatemala); mostly } \\
\text { cultivated and naturalized } \\
\text { elsewhere in tropical and } \\
\text { subtropical regions }\end{array}$ & & $x$ & & & $\begin{array}{l}\text { Field work } \\
\text { (JDN) }\end{array}$ & JDN: CBNM, MO \\
\hline Asystasia sp. & Acanthaceae & $\begin{array}{l}\text { Ch suff } \\
\text { caesp }\end{array}$ & $?$ & & $x$ & $x$ & & $\begin{array}{l}\text { Field work } \\
\text { (JDN, GLO) }\end{array}$ & $\begin{array}{l}\text { JDN: CBNM, MO; GLO: } \\
\text { CBNM }\end{array}$ \\
\hline $\begin{array}{l}\text { Avicennia marina } \\
\text { (Forssk.) Vierh. }\end{array}$ & Avicenniaceae & P scap & Indo-Pacific & $x$ & & & & $\begin{array}{l}\text { Bibliography } \\
\text { (EUR) }\end{array}$ & EUR: CBNM, MO, P \\
\hline
\end{tabular}




\begin{tabular}{|c|c|c|c|c|c|c|c|c|c|}
\hline \multirow[t]{2}{*}{ Scientific name } & \multirow[t]{2}{*}{ Family } & \multirow{2}{*}{$\begin{array}{l}\text { Life } \\
\text { forms }\end{array}$} & \multirow{2}{*}{$\begin{array}{l}\text { General status and } \\
\text { distribution range }\end{array}$} & \multicolumn{4}{|c|}{ Presence } & \multirow{2}{*}{$\begin{array}{c}\text { First record } \\
\text { source from } \\
\text { (name of the } \\
\text { territory) }\end{array}$} & \multirow{2}{*}{$\begin{array}{l}\text { Voucher herbarium } \\
\text { specimens (name of the } \\
\text { territory and acronym of } \\
\text { the herbarium) }\end{array}$} \\
\hline & & & & $\begin{array}{l}\text { Europ } \\
\text { a } \\
\text { (EUR) }\end{array}$ & $\begin{array}{l}\text { Juan } \\
\text { de } \\
\text { Nova } \\
\text { (JDN) }\end{array}$ & $\begin{array}{l}\text { Iles } \\
\text { Glorieuses } \\
\text { (GLO) }\end{array}$ & $\begin{array}{l}\text { Tromelin } \\
\text { (TRO) }\end{array}$ & & \\
\hline $\begin{array}{l}\text { Bambusa vulgaris } \\
\text { Schrad. ex J.C. } \\
\text { Wendl. var. vittata } \\
\text { Rivière \& C. Rivière }\end{array}$ & Poaceae & P gram & $\begin{array}{l}\text { Tropical Asia; mostly } \\
\text { cultivated and naturalized in } \\
\text { tropical regions }\end{array}$ & & $x$ & & & $\begin{array}{l}\text { Field work } \\
\text { (JDN) }\end{array}$ & \\
\hline $\begin{array}{l}\text { Boerhavia coccinea } \\
\text { Mill. }\end{array}$ & Nyctaginaceae & $\begin{array}{l}\text { Ch herb } \\
\text { rept }\end{array}$ & Pantropical & $x$ & $x$ & & & $\begin{array}{l}\text { Field work } \\
\text { (EUR, JDN) }\end{array}$ & $\begin{array}{l}\text { EUR: CBNM, MO, P; JDN: } \\
\text { CBNM, MO, P }\end{array}$ \\
\hline Boerhavia diffusa $\mathrm{L}$. & Nyctaginaceae & $\begin{array}{l}\text { Ch herb } \\
\text { caesp }\end{array}$ & Pantropical & & & $x$ & & $\begin{array}{l}\text { Bibliography } \\
\text { (GLO) }\end{array}$ & GLO: CBNM \\
\hline Boerhavia erecta $\mathrm{L}$. & Nyctaginaceae & $\begin{array}{l}\text { Ch herb } \\
\text { caesp }\end{array}$ & $\begin{array}{l}\text { Pantropical; probably native } \\
\text { from tropical America }\end{array}$ & $x$ & $x$ & $x$ & & $\begin{array}{l}\text { Field work } \\
\text { (EUR, JDN, } \\
\text { GLO) }\end{array}$ & $\begin{array}{l}\text { JDN: CBNM, MO; GLO: } \\
\text { CBNM, MO, P }\end{array}$ \\
\hline Boerhavia repens L. & Nyctaginaceae & $\begin{array}{l}\text { Ch herb } \\
\text { rept }\end{array}$ & Pantropical & $x$ & & & & $\begin{array}{l}\text { Bibliography } \\
\text { (EUR) }\end{array}$ & EUR: CBNM, MO, P \\
\hline $\begin{array}{l}\text { Boerhavia sp. nov. } 1 \\
\text { ? }\end{array}$ & Nyctaginaceae & $\begin{array}{l}\text { Ch herb } \\
\text { rept }\end{array}$ & West of the Indian Ocean & & $x$ & $x$ & & $\begin{array}{l}\text { Bibliography } \\
\text { (GLO); Field } \\
\text { work (JDN) }\end{array}$ & $\begin{array}{l}\text { JDN: CBNM, MO, P; GLO: } \\
\text { CBNM, MO, P }\end{array}$ \\
\hline $\begin{array}{l}\text { Boerhavia sp. nov. } 2 \\
\text { ? }\end{array}$ & Nyctaginaceae & $\begin{array}{l}\text { Ch herb } \\
\text { rept }\end{array}$ & Tromelin & & & & $x$ & $\begin{array}{l}\text { Bibliography } \\
\text { (TRO) }\end{array}$ & TRO: CBNM, MO, P \\
\hline $\begin{array}{l}\text { Bothriochloa pertusa } \\
\text { (L.) A. Camus }\end{array}$ & Poaceae & $\mathrm{H}$ rept & $\begin{array}{l}\text { Warm regions from Africa, } \\
\text { India and China; introduced } \\
\text { and naturalized elsewhere }\end{array}$ & $x$ & & $x$ & & $\begin{array}{l}\text { Field work } \\
\text { (EUR, GLO) }\end{array}$ & $\begin{array}{l}\text { EUR: CBNM, MO, P; GLO: } \\
\text { CBNM, MO, P }\end{array}$ \\
\hline $\begin{array}{l}\text { Bougainvillea } x \\
\text { buttiana Holttum \& } \\
\text { Standl. }\end{array}$ & Nyctaginaceae & $d P L$ & $\begin{array}{l}\text { Hybrid of horticultural origin, } \\
\text { cultivated in warm regions }\end{array}$ & $x$ & $x$ & & & $\begin{array}{l}\text { Bibliography } \\
\text { (EUR); Field } \\
\text { work (JDN) }\end{array}$ & JDN: CBNM, MO \\
\hline $\begin{array}{l}\text { Bruguiera } \\
\text { gymnorhiza (L.) } \\
\text { Savigny }\end{array}$ & Rhizophoraceae & P scap & $\begin{array}{l}\text { Indo-Pacific coastlines (from } \\
\text { East Africa to Fiji) }\end{array}$ & $x$ & & & & $\begin{array}{l}\text { Bibliography } \\
\text { (EUR) }\end{array}$ & EUR: CBNM, MO, P \\
\hline
\end{tabular}




\begin{tabular}{|c|c|c|c|c|c|c|c|c|c|}
\hline \multirow[t]{2}{*}{ Scientific name } & \multirow[t]{2}{*}{ Family } & \multirow{2}{*}{$\begin{array}{l}\text { Life } \\
\text { forms }\end{array}$} & \multirow{2}{*}{$\begin{array}{l}\text { General status and } \\
\text { distribution range }\end{array}$} & \multicolumn{4}{|c|}{ Presence } & \multirow{2}{*}{$\begin{array}{l}\text { First record } \\
\text { source from } \\
\text { (name of the } \\
\text { territory) }\end{array}$} & \multirow{2}{*}{$\begin{array}{l}\text { Voucher herbarium } \\
\text { specimens (name of the } \\
\text { territory and acronym of } \\
\text { the herbarium) }\end{array}$} \\
\hline & & & & $\begin{array}{l}\text { Europ } \\
\quad a \\
\text { (EUR) }\end{array}$ & $\begin{array}{l}\text { Juan } \\
\text { de } \\
\text { Nova } \\
\text { (JDN) }\end{array}$ & $\begin{array}{l}\text { Iles } \\
\text { Glorieuses } \\
\text { (GLO) }\end{array}$ & $\begin{array}{l}\text { Tromelin } \\
\text { (TRO) }\end{array}$ & & \\
\hline $\begin{array}{l}\text { Bulbostylis basalis } \\
\text { Fosberg }\end{array}$ & Cyperaceae & H caesp & Seychelles (Aldabra) & & & $x$ & & $\begin{array}{l}\text { Field work } \\
\text { (GLO) }\end{array}$ & GLO: CBNM, MO, P \\
\hline $\begin{array}{l}\text { Bulbostylis hispidula } \\
\text { (Vahl) R.W. Haines } \\
\text { subsp. hispidula }\end{array}$ & Cyperaceae & T caesp & $\begin{array}{l}\text { Africa, Northern America, } \\
\text { Southern America, } \\
\text { Madagascar, Seychelles }\end{array}$ & & $x$ & & & $\begin{array}{l}\text { Field work } \\
\text { (JDN) }\end{array}$ & JDN: CBNM, MO, P \\
\hline $\begin{array}{l}\text { Calophyllum } \\
\text { inophyllum L. }\end{array}$ & Calophyllaceae & P scap & $\begin{array}{l}\text { East Africa (coastlines), from } \\
\text { Madagascar to Pacific }\end{array}$ & & $x$ & $x$ & & $\begin{array}{l}\text { Bibliography } \\
\text { (GLO); Field } \\
\text { work (JDN) }\end{array}$ & $\begin{array}{l}\text { JDN: CBNM, MO; GLO: } \\
\text { CBNM, MO, P }\end{array}$ \\
\hline $\begin{array}{l}\text { Canavalia rosea (Sw.) } \\
\text { DC. }\end{array}$ & Fabaceae & st PL & $\begin{array}{l}\text { Tropical and subtropical } \\
\text { costlines }\end{array}$ & & $x$ & $x$ & & $\begin{array}{l}\text { Bibliography } \\
\text { (JDN); Field } \\
\text { work (GLO) }\end{array}$ & JDN: CBNM, MO, P \\
\hline $\begin{array}{l}\text { Capparis cartilaginea } \\
\text { Decne. }\end{array}$ & Capparaceae & P caesp & $\begin{array}{l}\text { Tropical and subtropical Africa } \\
\text { (East and North Africa) and } \\
\text { Asia (Middle East and South } \\
\text { West Asia) }\end{array}$ & $x$ & $x$ & & & $\begin{array}{l}\text { Bibliography } \\
\text { (EUR); Field } \\
\text { work (JDN) }\end{array}$ & $\begin{array}{l}\text { EUR: CBNM, MO, P; JDN: } \\
\text { CBNM, MO, P }\end{array}$ \\
\hline $\begin{array}{l}\text { Capsicum frutescens } \\
\text { L. }\end{array}$ & Solanaceae & $\begin{array}{l}\text { T caesp } \\
\text { / P herb }\end{array}$ & America & $x$ & & $x$ & & $\begin{array}{l}\text { Bibliography } \\
\text { (EUR); Field } \\
\text { work (GLO) }\end{array}$ & GLO: CBNM, MO, P \\
\hline Carica papaya L. & Caricaceae & P scap & $\begin{array}{l}\text { Tropical America (from Florida } \\
\text { to Northern Argentina); } \\
\text { mostly cultivated in tropical } \\
\text { regions and often naturalized }\end{array}$ & & & $x$ & $x$ & $\begin{array}{l}\text { Bibliography } \\
\text { (GLO, TRO) }\end{array}$ & GLO: CBNM, MO, P \\
\hline $\begin{array}{l}\text { Cascabela thevetia } \\
\text { (L.) Lippold }\end{array}$ & Apocynaceae & P caesp & $\begin{array}{l}\text { Topical America; widely } \\
\text { cultivated in tropical regions }\end{array}$ & & $x$ & & & $\begin{array}{l}\text { Field work } \\
\text { (JDN) }\end{array}$ & \\
\hline Cassytha filiformis L. & Lauraceae & $\mathrm{EL}$ & Pantropical & & $x$ & $x$ & & $\begin{array}{l}\text { Bibliography } \\
\text { (GLO); Field } \\
\text { work (JDN) }\end{array}$ & $\begin{array}{l}\text { JDN: CBNM, MO, P; GLO: } \\
\text { CBNM, MO, P }\end{array}$ \\
\hline
\end{tabular}




\begin{tabular}{|c|c|c|c|c|c|c|c|c|c|}
\hline \multirow[t]{2}{*}{ Scientific name } & \multirow[t]{2}{*}{ Family } & \multirow{2}{*}{$\begin{array}{l}\text { Life } \\
\text { forms }\end{array}$} & \multirow{2}{*}{$\begin{array}{l}\text { General status and } \\
\text { distribution range }\end{array}$} & \multicolumn{4}{|c|}{ Presence } & \multirow{2}{*}{$\begin{array}{l}\text { First record } \\
\text { source from } \\
\text { (name of the } \\
\text { territory) }\end{array}$} & \multirow{2}{*}{$\begin{array}{l}\text { Voucher herbarium } \\
\text { specimens (name of the } \\
\text { territory and acronym o } \\
\text { the herbarium) }\end{array}$} \\
\hline & & & & $\begin{array}{l}\text { Europ } \\
\quad \text { a } \\
\text { (EUR) }\end{array}$ & $\begin{array}{l}\text { Juan } \\
\text { de } \\
\text { Nova } \\
\text { (JDN) }\end{array}$ & $\begin{array}{l}\text { Iles } \\
\text { Glorieuses } \\
\text { (GLO) }\end{array}$ & $\begin{array}{l}\text { Tromelin } \\
\text { (TRO) }\end{array}$ & & \\
\hline $\begin{array}{l}\text { Casuarina } \\
\text { equisetifolia } \mathrm{L} .\end{array}$ & Casuarinaceae & P scap & $\begin{array}{l}\text { Indo-Pacific, Australian and } \\
\text { New Zealand coastlines; } \\
\text { mostly cultivated in tropical } \\
\text { regions and often naturalized }\end{array}$ & $x$ & $x$ & $x$ & $x$ & $\begin{array}{l}\text { Bibliography } \\
\text { (EUR, JDN, } \\
\text { GLO, TRO) }\end{array}$ & $\begin{array}{l}\text { EUR: CBNM, MO, P; JDN: } \\
\text { CBNM, MO; GLO: CBNM, } \\
\text { MO, P }\end{array}$ \\
\hline $\begin{array}{l}\text { Catharanthus roseus } \\
\text { (L.) G. Don }\end{array}$ & Apocynaceae & $\begin{array}{l}\text { Ch suff } \\
\text { caesp }\end{array}$ & Madagascar & & $x$ & $x$ & & $\begin{array}{l}\text { Bibliography } \\
\text { (GLO); Field } \\
\text { work (JDN) }\end{array}$ & $\begin{array}{l}\text { JDN: CBNM, MO, P; GLO: } \\
\text { CBNM, MO, P }\end{array}$ \\
\hline $\begin{array}{l}\text { Ceiba pentandra (L.) } \\
\text { Gaertn. }\end{array}$ & Malvaceae & P scap & Tropical America & & $x$ & & & $\begin{array}{l}\text { Field work } \\
\text { (JDN) }\end{array}$ & JDN: CBNM, MO, P \\
\hline $\begin{array}{l}\text { Celosia spicata } \\
\text { (Thouars) Spreng. }\end{array}$ & Amaranthaceae & $d P L$ & $\begin{array}{l}\text { Madagascar, Comoros and } \\
\text { Seychelles (Aldabra) }\end{array}$ & & $x$ & $x$ & & $\begin{array}{l}\text { Field work } \\
\text { (JDN, GLO) }\end{array}$ & $\begin{array}{l}\text { JDN: CBNM , MO, P; } \\
\text { GLO: CBNM, MO, P }\end{array}$ \\
\hline Cenchrus echinatus L. & Poaceae & T caesp & $\begin{array}{l}\text { Tropical America, became } \\
\text { pantropical }\end{array}$ & & $x$ & $x$ & & $\begin{array}{l}\text { Bibliography } \\
\text { (GLO); Field } \\
\text { work (JDN) }\end{array}$ & $\begin{array}{l}\text { JDN: CBNM, MO; GLO: } \\
\text { CBNM, MO, P }\end{array}$ \\
\hline $\begin{array}{l}\text { Cenchrus } \\
\text { polystachios (L.) } \\
\text { Morrone }\end{array}$ & Poaceae & $\begin{array}{l}\text { H caesp } \\
\text { / T } \\
\text { caesp / } \\
\text { P gram }\end{array}$ & Pantropical & & & $x$ & & $\begin{array}{l}\text { Field work } \\
\text { (GLO) }\end{array}$ & GLO: CBNM, MO, P \\
\hline $\begin{array}{l}\text { Ceriops tagal (Perr.) } \\
\text { C.B. Rob. }\end{array}$ & Rhizophoraceae & P scap & $\begin{array}{l}\text { Indo-Pacific coastlines (from } \\
\text { East Africa to Melanesia) }\end{array}$ & $x$ & & & & $\begin{array}{l}\text { Bibliography } \\
\text { (EUR) }\end{array}$ & EUR: CBNM, MO, P \\
\hline $\begin{array}{l}\text { Cheirolaena linearis } \\
\text { Benth }\end{array}$ & Malvaceae & T scap & Madagascar & & $x$ & & & $\begin{array}{l}\text { Field work } \\
\text { (JDN) }\end{array}$ & JDN: CBNM, MO, P \\
\hline Chloris barbata Sw. & Poaceae & $\begin{array}{l}\text { T caesp } \\
\text { / H } \\
\text { caesp }\end{array}$ & Tropical cosmopolitan & $x$ & & $x$ & & $\begin{array}{l}\text { Field work } \\
\text { (EUR, GLO) }\end{array}$ & $\begin{array}{l}\text { EUR: CBNM, MO, P; GLO: } \\
\text { CBNM, MO, P }\end{array}$ \\
\hline $\begin{array}{l}\text { Christella cf. dentata } \\
\text { (Forssk.) Brownsey \& } \\
\text { Jermy }\end{array}$ & $\begin{array}{l}\text { Thelypteridacea } \\
\text { e }\end{array}$ & $\begin{array}{l}\text { Ch herb } \\
\text { caesp }\end{array}$ & $\begin{array}{l}\text { Paleotropical and } \\
\text { paleosubtropical; introduced and } \\
\text { naturalized elsewhere (America) }\end{array}$ & & & $x$ & & $\begin{array}{l}\text { Field work } \\
\text { (GLO) }\end{array}$ & GLO: CBNM \\
\hline
\end{tabular}




\begin{tabular}{|c|c|c|c|c|c|c|c|c|c|}
\hline \multirow[t]{2}{*}{ Scientific name } & \multirow[t]{2}{*}{ Family } & \multirow{2}{*}{$\begin{array}{l}\text { Life } \\
\text { forms }\end{array}$} & \multirow{2}{*}{$\begin{array}{l}\text { General status and } \\
\text { distribution range }\end{array}$} & \multicolumn{4}{|c|}{ Presence } & \multirow{2}{*}{$\begin{array}{l}\text { First record } \\
\text { source from } \\
\text { (name of the } \\
\text { territory) }\end{array}$} & \multirow{2}{*}{$\begin{array}{l}\text { Voucher herbarium } \\
\text { specimens (name of the } \\
\text { territory and acronym of } \\
\text { the herbarium) }\end{array}$} \\
\hline & & & & $\begin{array}{l}\text { Europ } \\
\text { a } \\
\text { (EUR) }\end{array}$ & $\begin{array}{l}\text { Juan } \\
\text { de } \\
\text { Nova } \\
\text { (JDN) }\end{array}$ & $\begin{array}{l}\text { Iles } \\
\text { Glorieuses } \\
\text { (GLO) }\end{array}$ & $\begin{array}{l}\text { Tromelin } \\
\text { (TRO) }\end{array}$ & & \\
\hline $\begin{array}{l}\text { Citrus aurantiifolia } \\
\text { (Christm.) Swingle }\end{array}$ & Rutaceae & P caesp & $\begin{array}{l}\text { South East of Asia (or } \\
\text { Indonesia), mostly cultivated } \\
\text { in tropical and subtropical } \\
\text { regions }\end{array}$ & & $x$ & & & $\begin{array}{l}\text { Field work } \\
\text { (JDN) }\end{array}$ & JDN: CBNM \\
\hline $\begin{array}{l}\text { Cleome grandidieri } \\
\text { Baill. }\end{array}$ & Cleomaceae & T caesp & Madagascar & & $\mathrm{x}$ & & & $\begin{array}{l}\text { Field work } \\
\text { (JDN) }\end{array}$ & JDN: CBNM, MO, P \\
\hline $\begin{array}{l}\text { Cleome strigosa } \\
\text { (Boj.) Oliv. }\end{array}$ & Cleomaceae & T caesp & $\begin{array}{l}\text { East African coastlines, coral } \\
\text { Seychelles, Zanzibar, Pemba }\end{array}$ & & $\mathrm{x}$ & $x$ & & $\begin{array}{l}\text { Bibliography } \\
\text { (GLO); Field } \\
\text { work (JDN) }\end{array}$ & $\begin{array}{l}\text { JDN: CBNM, MO; GLO: } \\
\text { CBNM, MO, P }\end{array}$ \\
\hline Cocos nucifera L. & Arecaceae & P ros & $\begin{array}{l}\text { Supposed native from central } \\
\text { Malaysia; widespread in } \\
\text { tropical regions (became } \\
\text { pantropical); cultivated in } \\
\text { tropical regions and } \\
\text { naturalised }\end{array}$ & $x$ & $\mathrm{x}$ & $x$ & $\mathrm{x}$ & $\begin{array}{l}\text { Bibliography } \\
\text { (EUR, JDN, } \\
\text { GLO, TRO) }\end{array}$ & \\
\hline $\begin{array}{l}\text { Colubrina asiatica (L.) } \\
\text { Brongn. }\end{array}$ & Rhamnaceae & P caesp & $\begin{array}{l}\text { Asia, Malaysia, Pacific and } \\
\text { East Africa coastlines, } \\
\text { Madagascar }\end{array}$ & & $x$ & $x$ & & $\begin{array}{l}\text { Bibliography } \\
\text { (GLO); Field } \\
\text { work (JDN) }\end{array}$ & $\begin{array}{l}\text { JDN: CBNM, MO, P; GLO: } \\
\text { CBNM, MO, P }\end{array}$ \\
\hline $\begin{array}{l}\text { Commelina } \\
\text { benghalensis } \mathrm{L} .\end{array}$ & Commelinaceae & $\begin{array}{l}\text { Ch herb } \\
\text { rept }\end{array}$ & $\begin{array}{l}\text { Tropical Africa, South Africa, } \\
\text { Madagascar, India and South } \\
\text { East Asia }\end{array}$ & & & $x$ & & $\begin{array}{l}\text { Field work } \\
\text { (GLO) }\end{array}$ & GLO: CBNM, MO, P \\
\hline $\begin{array}{l}\text { Commelina forskalaei } \\
\text { Vahl }\end{array}$ & Commelinaceae & $\begin{array}{l}\text { Ch herb } \\
\text { rept }\end{array}$ & $\begin{array}{l}\text { Africa, Arabian peninsula, } \\
\text { South India, China }\end{array}$ & & $x$ & & & $\begin{array}{l}\text { Bibliography } \\
\text { (JDN) }\end{array}$ & JDN: CBNM, MO, P \\
\hline $\begin{array}{l}\text { Commicarpus } \\
\text { plumbagineus (Cav.) } \\
\text { Standl. }\end{array}$ & Nyctaginaceae & $d P L$ & South and tropical Africa, Asia & & $\mathrm{x}$ & $\mathrm{x}$ & & $\begin{array}{l}\text { Field work } \\
\text { (JDN, GLO) }\end{array}$ & $\begin{array}{l}\text { JDN: CBNM; GLO: CBNM, } \\
\text { MO, P }\end{array}$ \\
\hline
\end{tabular}




\begin{tabular}{|c|c|c|c|c|c|c|c|c|c|}
\hline \multirow[t]{2}{*}{ Scientific name } & \multirow[t]{2}{*}{ Family } & \multirow{2}{*}{$\begin{array}{l}\text { Life } \\
\text { forms }\end{array}$} & \multirow{2}{*}{$\begin{array}{l}\text { General status and } \\
\text { distribution range }\end{array}$} & \multicolumn{4}{|c|}{ Presence } & \multirow{2}{*}{$\begin{array}{l}\text { First record } \\
\text { source from } \\
\text { (name of the } \\
\text { territory) }\end{array}$} & \multirow{2}{*}{$\begin{array}{l}\text { Voucher herbarium } \\
\text { specimens (name of the } \\
\text { territory and acronym of } \\
\text { the herbarium) }\end{array}$} \\
\hline & & & & $\begin{array}{l}\text { Europ } \\
\quad \text { a } \\
\text { (EUR) }\end{array}$ & $\begin{array}{l}\text { Juan } \\
\text { de } \\
\text { Nova } \\
\text { (JDN) }\end{array}$ & $\begin{array}{l}\text { Iles } \\
\text { Glorieuses } \\
\text { (GLO) }\end{array}$ & $\begin{array}{l}\text { Tromelin } \\
\text { (TRO) }\end{array}$ & & \\
\hline $\begin{array}{l}\text { Consolea falcata } \\
\text { (Ekman \& Werderm.) } \\
\text { F.M. Knuth }\end{array}$ & Cactaceae & P succ & North West Haiti & & $x$ & & $x$ & $\begin{array}{l}\text { Field work } \\
\text { (JDN, TRO) }\end{array}$ & \\
\hline $\begin{array}{l}\text { Corchorus aestuans } \\
\text { L. }\end{array}$ & Malvaceae & T caesp & Pantropical & & $x$ & $X$ & & $\begin{array}{l}\text { Field work } \\
\text { (JDN, GLO) }\end{array}$ & $\begin{array}{l}\text { JDN: CBNM, MO; GLO: } \\
\text { CBNM }\end{array}$ \\
\hline $\begin{array}{l}\text { Corchorus trilocularis } \\
\text { L. }\end{array}$ & Malvaceae & T scap & $\begin{array}{l}\text { Paleotropical to } \\
\text { paleosubtropical, became a } \\
\text { pantropical weed }\end{array}$ & $x$ & & & & $\begin{array}{l}\text { Field work } \\
\text { (EUR) }\end{array}$ & EUR: CBNM, MO, P \\
\hline $\begin{array}{l}\text { Cordia subcordata } \\
\text { Lam. }\end{array}$ & Boraginaceae & P scap & Indo-Pacific coastlines & $x$ & $x$ & $x$ & & $\begin{array}{l}\text { Bibliography } \\
\text { (EUR, GLO); } \\
\text { Field work } \\
\text { (JDN) }\end{array}$ & $\begin{array}{l}\text { EUR: CBNM, MO, P; JDN: } \\
\text { CBNM, MO; GLO: CBNM, } \\
\text { MO, P }\end{array}$ \\
\hline $\begin{array}{l}\text { Crotalaria edmundi- } \\
\text { bakeri R. Vig. }\end{array}$ & Fabaceae & $\begin{array}{l}\text { T caesp } \\
\text { / Ch suff } \\
\text { caesp }\end{array}$ & $\begin{array}{l}\text { East African coastlines, from } \\
\text { Mozambique to Somalia, } \\
\text { Comoros, Aldabra }\end{array}$ & & $x$ & & & $\begin{array}{l}\text { Field work } \\
\text { (JDN) }\end{array}$ & JDN: CBNM, MO, P \\
\hline $\begin{array}{l}\text { Cucumis melo L. } \\
\text { subsp. agrestis } \\
\text { (Naudin) Pangalo var. } \\
\text { agrestis }\end{array}$ & Cucurbitaceae & el TL & Africa & $x$ & $x$ & $x$ & & $\begin{array}{l}\text { Bibliography } \\
\text { (EUR, GLO); } \\
\text { Field work } \\
\text { (JDN) }\end{array}$ & $\begin{array}{l}\text { EUR: CBNM, MO, P; JDN: } \\
\text { CBNM, MO; GLO: CBNM, } \\
\text { MO }\end{array}$ \\
\hline $\begin{array}{l}\text { Cyanthillium } \\
\text { cinereum (L.) H. Rob. }\end{array}$ & Asteraceae & T sem & Tropical Africa and Asia & & & $x$ & & $\begin{array}{l}\text { Field work } \\
\text { (GLO) }\end{array}$ & GLO: CBNM, MO, P \\
\hline $\begin{array}{l}\text { Cynanchum } \\
\text { luteifluens (Jum. \& H. } \\
\text { Perrier) Desc. }\end{array}$ & Apocynaceae & st PL & Madagascar & $x$ & & & & $\begin{array}{l}\text { Bibliography } \\
\text { (EUR) }\end{array}$ & EUR: CBNM, MO, P \\
\hline $\begin{array}{l}\text { Cynodon dactylon (L.) } \\
\text { Pers. }\end{array}$ & Poaceae & $\begin{array}{l}\text { H rept / } \\
\text { c G rhiz }\end{array}$ & Cosmopolitan & $x$ & $x$ & & & $\begin{array}{l}\text { Field work } \\
\text { (EUR, JDN) }\end{array}$ & $\begin{array}{l}\text { EUR: CBNM, MO, P; JDN: } \\
\text { CBNM, MO, P }\end{array}$ \\
\hline Cynodon sp. & Poaceae & $\begin{array}{l}\text { H rept / } \\
\text { c G rhiz }\end{array}$ & ? & & $x$ & & & $\begin{array}{l}\text { Field work } \\
\text { (JDN) }\end{array}$ & JDN: CBNM, MO, P \\
\hline
\end{tabular}




\begin{tabular}{|c|c|c|c|c|c|c|c|c|c|}
\hline \multirow[t]{2}{*}{ Scientific name } & \multirow[t]{2}{*}{ Family } & \multirow{2}{*}{$\begin{array}{l}\text { Life } \\
\text { forms }\end{array}$} & \multirow{2}{*}{$\begin{array}{l}\text { General status and } \\
\text { distribution range }\end{array}$} & \multicolumn{4}{|c|}{ Presence } & \multirow{2}{*}{$\begin{array}{l}\text { First record } \\
\text { source from } \\
\text { (name of the } \\
\text { territory) }\end{array}$} & \multirow{2}{*}{$\begin{array}{l}\text { Voucher herbarium } \\
\text { specimens (name of the } \\
\text { territory and acronym o } \\
\text { the herbarium) }\end{array}$} \\
\hline & & & & $\begin{array}{l}\text { Europ } \\
\quad a \\
\text { (EUR) }\end{array}$ & $\begin{array}{l}\text { Juan } \\
\text { de } \\
\text { Nova } \\
\text { (JDN) }\end{array}$ & $\begin{array}{l}\text { Iles } \\
\text { Glorieuses } \\
\text { (GLO) }\end{array}$ & $\begin{array}{l}\text { Tromelin } \\
\text { (TRO) }\end{array}$ & & \\
\hline $\begin{array}{l}\text { Cyperus dubius } \\
\text { Rottb. }\end{array}$ & Cyperaceae & H caesp & $\begin{array}{l}\text { Paleotropical and subtropical } \\
\text { (from West Africa to Asia and } \\
\text { Philippines) }\end{array}$ & & & $x$ & & $\begin{array}{l}\text { Field work } \\
\text { (GLO) }\end{array}$ & GLO: CBNM, MO, P \\
\hline Cyperus ligularis L. & Cyperaceae & H caesp & Pantropical & & & $x$ & & $\begin{array}{l}\text { Field work } \\
\text { (GLO) }\end{array}$ & GLO: CBNM, MO, P \\
\hline Cyperus rotundus L. & Cyperaceae & $\begin{array}{l}\text { H caesp } \\
\text { / c G } \\
\text { rhiz / Ch } \\
\text { herb } \\
\text { caesp }\end{array}$ & $\begin{array}{l}\text { Pantropical and } \\
\text { pansubtropical }\end{array}$ & $x$ & $x$ & $x$ & & $\begin{array}{l}\text { Bibliography } \\
\text { (JDN, GLO); } \\
\text { Field work } \\
\text { (EUR) }\end{array}$ & $\begin{array}{l}\text { EUR: CBNM, MO, P; JDN: } \\
\text { CBNM, MO, P; GLO: } \\
\text { CBNM, MO, P }\end{array}$ \\
\hline $\begin{array}{l}\text { Dactyloctenium } \\
\text { aegyptium (L.) Willd. }\end{array}$ & Poaceae & T caesp & $\begin{array}{l}\text { Pantropical and } \\
\text { pansubtropical }\end{array}$ & $x$ & $x$ & & & $\begin{array}{l}\text { Bibliography } \\
\text { (JDN); Field } \\
\text { work (EUR) }\end{array}$ & $\begin{array}{l}\text { EUR: CBNM, MO, P; JDN: } \\
\text { CBNM, MO, P }\end{array}$ \\
\hline $\begin{array}{l}\text { Dactyloctenium } \\
\text { aristatum Link }\end{array}$ & Poaceae & T caesp & $\begin{array}{l}\text { Pakistan, Kenya to Sudan, } \\
\text { Egypt, Arabia to India }\end{array}$ & & & & $x$ & $\begin{array}{l}\text { Field work } \\
\text { (TRO) }\end{array}$ & TRO: CBNM, MO, P \\
\hline $\begin{array}{l}\text { Dactyloctenium } \\
\text { capitatum A. Camus }\end{array}$ & Poaceae & $\begin{array}{l}\text { T caesp } \\
\text { / H rept }\end{array}$ & Madagascar & $x$ & & & & $\begin{array}{l}\text { Bibliography } \\
\text { (EUR) }\end{array}$ & EUR: CBNM, MO, P \\
\hline $\begin{array}{l}\text { Dactyloctenium } \\
\text { ctenioides (Steud.) } \\
\text { Lorch ex Bosser }\end{array}$ & Poaceae & T caesp & $\begin{array}{l}\text { East African, Madagascarn } \\
\text { Mascarene islands and } \\
\text { Seychelles coastlines }\end{array}$ & $x$ & $x$ & $x$ & & $\begin{array}{l}\text { Bibliography } \\
\text { (GLO); Field } \\
\text { work (EUR, } \\
\text { JDN) }\end{array}$ & $\begin{array}{l}\text { EUR: CBNM, MO, P; JDN: } \\
\text { CBNM, MO, P; GLO: } \\
\text { CBNM, MO, P }\end{array}$ \\
\hline $\begin{array}{l}\text { Dactyloctenium } \\
\text { geminatum Hack. }\end{array}$ & Poaceae & H rept & East and South East Africa & & $x$ & & & $\begin{array}{l}\text { Field work } \\
\text { (JDN) }\end{array}$ & JDN: CBNM, MO, P \\
\hline $\begin{array}{l}\text { Daknopholis boivinii } \\
\text { (A. Camus) Clayton }\end{array}$ & Poaceae & Trept & $\begin{array}{l}\text { East Africa, Madagascar, coral } \\
\text { Seychelles (Aldabra, } \\
\text { Cosmoledo); introduced } \\
\text { elsewhere (Reunion) }\end{array}$ & $x$ & $x$ & $x$ & & $\begin{array}{l}\text { Field work } \\
\text { (EUR, JDN, } \\
\text { GLO) }\end{array}$ & $\begin{array}{l}\text { EUR: CBNM, MO, P; JDN: } \\
\text { CBNM, MO, P; GLO: } \\
\text { CBNM, MO, P }\end{array}$ \\
\hline
\end{tabular}




\begin{tabular}{|c|c|c|c|c|c|c|c|c|c|}
\hline \multirow[t]{2}{*}{ Scientific name } & \multirow[t]{2}{*}{ Family } & \multirow{2}{*}{$\begin{array}{l}\text { Life } \\
\text { forms }\end{array}$} & \multirow{2}{*}{$\begin{array}{l}\text { General status and } \\
\text { distribution range }\end{array}$} & \multicolumn{4}{|c|}{ Presence } & \multirow{2}{*}{$\begin{array}{l}\text { First record } \\
\text { source from } \\
\text { (name of the } \\
\text { territory) }\end{array}$} & \multirow{2}{*}{$\begin{array}{l}\text { Voucher herbarium } \\
\text { specimens (name of the } \\
\text { territory and acronym of } \\
\text { the herbarium) }\end{array}$} \\
\hline & & & & $\begin{array}{l}\text { Europ } \\
\quad a \\
\text { (EUR) }\end{array}$ & $\begin{array}{l}\text { Juan } \\
\text { de } \\
\text { Nova } \\
\text { (JDN) }\end{array}$ & $\begin{array}{l}\text { Iles } \\
\text { Glorieuses } \\
\text { (GLO) }\end{array}$ & $\begin{array}{l}\text { Tromelin } \\
\text { (TRO) }\end{array}$ & & \\
\hline Datura innoxia Mill. & Solanaceae & T caesp & $\begin{array}{l}\text { Central America; naturalized } \\
\text { in tropical and subtropical } \\
\text { regions }\end{array}$ & & $x$ & $x$ & & $\begin{array}{l}\text { Field work } \\
\text { (JDN, GLO) }\end{array}$ & JDN: CBNM, MO, P \\
\hline $\begin{array}{l}\text { Delonix regia (Bojer) } \\
\text { Raf. }\end{array}$ & Fabaceae & P scap & Madagascar & $x$ & $x$ & & & $\begin{array}{l}\text { Bibliography } \\
\text { (EUR); Field } \\
\text { work (JDN) }\end{array}$ & EUR: CBNM, MO, P \\
\hline $\begin{array}{l}\text { Digitaria ciliaris } \\
\text { (Retz.) Koeler var. } \\
\text { chrysoblephara (Fig. } \\
\text { \& De Not.) }\end{array}$ & Poaceae & T caesp & $\begin{array}{l}\text { China, United-States of } \\
\text { America, Pakistan, } \\
\text { Madagascar, Southern Africa }\end{array}$ & $x$ & & & & $\begin{array}{l}\text { Field work } \\
\text { (EUR) }\end{array}$ & EUR: CBNM, MO, P \\
\hline $\begin{array}{l}\text { Digitaria horizontalis } \\
\text { Willd. }\end{array}$ & Poaceae & T caesp & $\begin{array}{l}\text { West tropical Africa and } \\
\text { tropical America; introduced } \\
\text { and naturalized elsewhere }\end{array}$ & & & $x$ & & $\begin{array}{l}\text { Field work } \\
\text { (GLO) }\end{array}$ & GLO: CBNM, MO, P \\
\hline $\begin{array}{l}\text { Digitaria setigera } \\
\text { Roth }\end{array}$ & Poaceae & T caesp & Paleotropical & & $x$ & & & $\begin{array}{l}\text { Field work } \\
\text { (JDN) }\end{array}$ & JDN: CBNM, MO, P \\
\hline Digitaria sp. & Poaceae & T caesp & $?$ & & & $x$ & & $\begin{array}{l}\text { Field work } \\
\text { (GLO) }\end{array}$ & GLO: CBNM \\
\hline $\begin{array}{l}\text { Disperis tripetaloides } \\
\text { (Thouars) Lindl. }\end{array}$ & Orchidaceae & c G bulb & $\begin{array}{l}\text { Madagascar, Comoros } \\
\text { (Mayotte), Mascarene islands } \\
\text { (Reunion, Mauritius and } \\
\text { Rodrigue), Seychelles }\end{array}$ & & & $x$ & & $\begin{array}{l}\text { Field work } \\
\text { (GLO) }\end{array}$ & GLO: CBNM, MO, P \\
\hline $\begin{array}{l}\text { Dodonaea viscosa } \\
\text { (L.) Jacq. }\end{array}$ & Sapindaceae & P caesp & Pantropical & & $x$ & $x$ & & $\begin{array}{l}\text { Field work } \\
\text { (JDN, GLO) }\end{array}$ & $\begin{array}{l}\text { JDN: CBNM, MO, P; GLO: } \\
\text { CBNM, MO }\end{array}$ \\
\hline $\begin{array}{l}\text { Dombeya greveana } \\
\text { Baill. var. } \\
\text { metameropsis } \\
\text { (Hochr.) Arènes }\end{array}$ & Malvaceae & P caesp & Madagascar & & $x$ & & & $\begin{array}{l}\text { Bibliography } \\
\text { (JDN) }\end{array}$ & JDN: CBNM, MO, P \\
\hline
\end{tabular}




\begin{tabular}{|c|c|c|c|c|c|c|c|c|c|}
\hline \multirow[t]{2}{*}{ Scientific name } & \multirow[t]{2}{*}{ Family } & \multirow{2}{*}{$\begin{array}{l}\text { Life } \\
\text { forms }\end{array}$} & \multirow{2}{*}{$\begin{array}{l}\text { General status and } \\
\text { distribution range }\end{array}$} & \multicolumn{4}{|c|}{ Presence } & \multirow{2}{*}{$\begin{array}{l}\text { First record } \\
\text { source from } \\
\text { (name of the } \\
\text { territory) }\end{array}$} & \multirow{2}{*}{$\begin{array}{l}\text { Voucher herbarium } \\
\text { specimens (name of the } \\
\text { territory and acronym of } \\
\text { the herbarium) }\end{array}$} \\
\hline & & & & $\begin{array}{l}\text { Europ } \\
\text { a } \\
\text { (EUR) }\end{array}$ & $\begin{array}{l}\text { Juan } \\
\text { de } \\
\text { Nova } \\
\text { (JDN) }\end{array}$ & $\begin{array}{l}\text { Iles } \\
\text { Glorieuses } \\
\text { (GLO) }\end{array}$ & $\begin{array}{l}\text { Tromelin } \\
\text { (TRO) }\end{array}$ & & \\
\hline $\begin{array}{l}\text { Eleusine indica (L.) } \\
\text { Gaertn. }\end{array}$ & Poaceae & $\begin{array}{l}\text { H caesp } \\
\text { / Ch } \\
\text { herb } \\
\text { caesp }\end{array}$ & $\begin{array}{l}\text { Subcosmopolitan tropical and } \\
\text { subtropical (introduced in } \\
\text { America) }\end{array}$ & & & $x$ & & $\begin{array}{l}\text { Field work } \\
\text { (GLO) }\end{array}$ & GLO: CBNM, MO, P \\
\hline $\begin{array}{l}\text { Enteropogon } \\
\text { sechellensis (Baker) } \\
\text { Benth. ex T. Durand } \\
\text { \& Schinz }\end{array}$ & Poaceae & H caesp & $\begin{array}{l}\text { East Africa, Madagascar, } \\
\text { Comoros, Seychelles }\end{array}$ & & & $x$ & & $\begin{array}{l}\text { Field work } \\
\text { (GLO) }\end{array}$ & GLO: CBNM, MO, P \\
\hline $\begin{array}{l}\text { Eragrostis capuronii } \\
\text { A. Camus }\end{array}$ & Poaceae & T caesp & Madagascar & $x$ & & & & $\begin{array}{l}\text { Field work } \\
\text { (EUR) }\end{array}$ & EUR: CBNM, MO, P \\
\hline $\begin{array}{l}\text { Eragrostis cilianensis } \\
\text { (All.) Vignolo ex } \\
\text { Janch. }\end{array}$ & Poaceae & T caesp & Subcosmopolitan & & $x$ & & & $\begin{array}{l}\text { Field work } \\
\text { (JDN) }\end{array}$ & JDN: CBNM, MO, P \\
\hline $\begin{array}{l}\text { Eragrostis ciliaris (L.) } \\
\mathrm{R} . \mathrm{Br} \text {. }\end{array}$ & Poaceae & T caesp & $\begin{array}{l}\text { Pantropical (supposed } \\
\text { introduced in America) }\end{array}$ & $\mathrm{X}$ & $\mathrm{x}$ & $x$ & & $\begin{array}{l}\text { Bibliography } \\
\text { (EUR); Field } \\
\text { work (JDN, } \\
\text { GLO) }\end{array}$ & $\begin{array}{l}\text { EUR: CBNM, MO, P; JDN: } \\
\text { CBNM, MO, P; GLO: } \\
\text { CBNM, MO, P }\end{array}$ \\
\hline Eragrostis minor Host & Poaceae & T caesp & Subcosmopolitan & & $x$ & & & $\begin{array}{l}\text { Field work } \\
\text { (JDN) }\end{array}$ & JDN: CBNM, MO, P \\
\hline $\begin{array}{l}\text { Eragrostis } \\
\text { subaequiglumis } \\
\text { Renvoize }\end{array}$ & Poaceae & T caesp & Seychelles & & & $x$ & & $\begin{array}{l}\text { Field work } \\
\text { (GLO) }\end{array}$ & GLO: CBNM, MO, P \\
\hline $\begin{array}{l}\text { Eriochloa fatmensis } \\
\text { (Hochst. \& Steud.) } \\
\text { Clayton }\end{array}$ & Poaceae & T caesp & $\begin{array}{l}\text { Tropical Africa, Southern } \\
\text { Africa and Asia temperate }\end{array}$ & & $x$ & & & $\begin{array}{l}\text { Field work } \\
\text { (JDN) }\end{array}$ & JDN: CBNM, MO, P \\
\hline
\end{tabular}




\begin{tabular}{|c|c|c|c|c|c|c|c|c|c|}
\hline \multirow[t]{2}{*}{ Scientific name } & \multirow[t]{2}{*}{ Family } & \multirow{2}{*}{$\begin{array}{l}\text { Life } \\
\text { forms }\end{array}$} & \multirow{2}{*}{$\begin{array}{l}\text { General status and } \\
\text { distribution range }\end{array}$} & \multicolumn{4}{|c|}{ Presence } & \multirow{2}{*}{$\begin{array}{l}\text { First record } \\
\text { source from } \\
\text { (name of the } \\
\text { territory) }\end{array}$} & \multirow{2}{*}{$\begin{array}{l}\text { Voucher herbarium } \\
\text { specimens (name of the } \\
\text { territory and acronym o } \\
\text { the herbarium) }\end{array}$} \\
\hline & & & & $\begin{array}{l}\text { Europ } \\
\quad a \\
\text { (EUR) }\end{array}$ & $\begin{array}{l}\text { Juan } \\
\text { de } \\
\text { Nova } \\
\text { (JDN) }\end{array}$ & $\begin{array}{l}\text { Iles } \\
\text { Glorieuses } \\
\text { (GLO) }\end{array}$ & $\begin{array}{l}\text { Tromelin } \\
\text { (TRO) }\end{array}$ & & \\
\hline Erythrina variegata $\mathrm{L}$. & Fabaceae & P scap & Indo-Pacific & & $x$ & $x$ & & $\begin{array}{l}\text { Field work } \\
\text { (JDN, GLO) }\end{array}$ & $\begin{array}{l}\text { JDN: CBNM, MO; GLO: } \\
\text { CBNM }\end{array}$ \\
\hline Euphorbia hirta L. & Euphorbiaceae & $\begin{array}{l}\text { T caesp } \\
\text { / Ch } \\
\text { herb } \\
\text { caesp }\end{array}$ & $\begin{array}{l}\text { Tropical America; widespread } \\
\text { in tropical and subtropical } \\
\text { regions }\end{array}$ & $x$ & $x$ & $x$ & $x$ & $\begin{array}{l}\text { Bibliography } \\
\text { (EUR, JDN, } \\
\text { GLO) ; Field } \\
\text { work (TRO) }\end{array}$ & $\begin{array}{l}\text { EUR: CBNM, MO, P; JDN: } \\
\text { CBNM; GLO: CBNM, MO, } \\
\text { P; TRO: CBNM, MO, P }\end{array}$ \\
\hline $\begin{array}{l}\text { Euphorbia mertonii } \\
\text { Fosberg }\end{array}$ & Euphorbiaceae & $\begin{array}{l}\text { Ch herb } \\
\text { rept }\end{array}$ & $\begin{array}{l}\text { Coral Seychelles (Aldabra) and } \\
\text { Madagascar }\end{array}$ & & $x$ & & & $\begin{array}{l}\text { Field work } \\
\text { (JDN) }\end{array}$ & JDN: CBNM \\
\hline $\begin{array}{l}\text { Euphorbia prostrata } \\
\text { Aiton }\end{array}$ & Euphorbiaceae & Trept & $\begin{array}{l}\text { Tropical and subtropical } \\
\text { America; widespread in } \\
\text { tropical, subtropical and warm } \\
\text { temperate regions }\end{array}$ & $x$ & $x$ & $x$ & $x$ & $\begin{array}{l}\text { Bibliography } \\
\text { (GLO); Field } \\
\text { work (EUR, } \\
\text { JDN, TRO) }\end{array}$ & $\begin{array}{l}\text { EUR: CBNM, MO, P; JDN: } \\
\text { CBNM, MO, P; GLO: } \\
\text { CBNM, MO, P; TRO: } \\
\text { CBNM, MO, P }\end{array}$ \\
\hline Euphorbia sp. nov.? & Euphorbiaceae & $\begin{array}{l}\text { Ch herb } \\
\text { caesp }\end{array}$ & Europa (endemic ?) & $x$ & & & & $\begin{array}{l}\text { Bibliography } \\
\text { (EUR) }\end{array}$ & EUR: CBNM, MO, P \\
\hline $\begin{array}{l}\text { Euphorbia stenoclada } \\
\text { Baill. }\end{array}$ & Euphorbiaceae & $\begin{array}{l}\text { P scap / } \\
\text { P succ }\end{array}$ & Madagascar & $x$ & $x$ & & & $\begin{array}{l}\text { Bibliography } \\
\text { (EUR, JDN) }\end{array}$ & \\
\hline $\begin{array}{l}\text { Euphorbia stoddartii } \\
\text { Fosberg }\end{array}$ & Euphorbiaceae & $\begin{array}{l}\text { Ch herb } \\
\text { rept }\end{array}$ & Coral Seychelles & & & $x$ & & $\begin{array}{l}\text { Bibliography } \\
\text { (GLO) }\end{array}$ & GLO: CBNM, MO, P \\
\hline $\begin{array}{l}\text { Euphorbia thymifolia } \\
\text { L. }\end{array}$ & Euphorbiaceae & $\begin{array}{l}\text { Trept / } \\
\text { Ch herb } \\
\text { rept }\end{array}$ & $\begin{array}{l}\text { Supposed native from Asia; } \\
\text { widespread in tropical regions }\end{array}$ & & $x$ & & & $\begin{array}{l}\text { Field work } \\
\text { (JDN) }\end{array}$ & JDN: CBNM, MO, P \\
\hline $\begin{array}{l}\text { Euphorbia } \\
\text { tithymaloides L. }\end{array}$ & Euphorbiaceae & $\begin{array}{l}\text { Ch st } \\
\text { succ }\end{array}$ & Florida to South America & & $x$ & $x$ & & $\begin{array}{l}\text { Field work } \\
\text { (JDN, GLO) }\end{array}$ & GLO: CBNM, MO, P \\
\hline $\begin{array}{l}\text { Evolvulus alsinoides } \\
\text { (L.) L. }\end{array}$ & Convolvulaceae & $\begin{array}{l}\text { Ch herb } \\
\text { rept / } \\
\text { Ch herb } \\
\text { caesp }\end{array}$ & $\begin{array}{l}\text { Pantropical (often considered } \\
\text { native to America) }\end{array}$ & & & $x$ & & $\begin{array}{l}\text { Field work } \\
\text { (GLO) }\end{array}$ & GLO: CBNM, MO, P \\
\hline
\end{tabular}




\begin{tabular}{|c|c|c|c|c|c|c|c|c|c|}
\hline \multirow[t]{2}{*}{ Scientific name } & \multirow[t]{2}{*}{ Family } & \multirow{2}{*}{$\begin{array}{l}\text { Life } \\
\text { forms }\end{array}$} & \multirow{2}{*}{$\begin{array}{l}\text { General status and } \\
\text { distribution range }\end{array}$} & \multicolumn{4}{|c|}{ Presence } & \multirow{2}{*}{$\begin{array}{l}\text { First record } \\
\text { source from } \\
\text { (name of the } \\
\text { territory) }\end{array}$} & \multirow{2}{*}{$\begin{array}{l}\text { Voucher herbarium } \\
\text { specimens (name of the } \\
\text { territory and acronym of } \\
\text { the herbarium) }\end{array}$} \\
\hline & & & & $\begin{array}{l}\text { Europ } \\
\quad a \\
\text { (EUR) }\end{array}$ & $\begin{array}{l}\text { Juan } \\
\text { de } \\
\text { Nova } \\
\text { (JDN) }\end{array}$ & $\begin{array}{l}\text { lles } \\
\text { Glorieuses } \\
\text { (GLO) }\end{array}$ & $\begin{array}{l}\text { Tromelin } \\
\text { (TRO) }\end{array}$ & & \\
\hline Ficus benghalensis L. & Moraceae & P scap & Asia & & $x$ & & & $\begin{array}{l}\text { Field work } \\
\text { (JDN) }\end{array}$ & JDN: CBNM \\
\hline Ficus grevei Baillon & Moraceae & P scap & Madagascar & & & $x$ & & $\begin{array}{l}\text { Bibliography } \\
\text { (GLO) }\end{array}$ & GLO: CBNM, MO, P \\
\hline $\begin{array}{l}\text { Ficus marmorata } \\
\text { Bojer ex Baker }\end{array}$ & Moraceae & P scap & Madagascar & $x$ & & & & $\begin{array}{l}\text { Bibliography } \\
\text { (EUR) }\end{array}$ & EUR: CBNM, MO, P \\
\hline Ficus sp. & Moraceae & P scap & $?$ & & & $x$ & & $\begin{array}{l}\text { Field work } \\
\text { (GLO) }\end{array}$ & GLO: CBNM, MO, P \\
\hline $\begin{array}{l}\text { Fimbristylis cymosa } \\
\text { R. Br. s. I. }\end{array}$ & Cyperaceae & $\begin{array}{l}\text { Ch herb } \\
\text { caesp }\end{array}$ & Pantropical & $x$ & & $x$ & & $\begin{array}{l}\text { Bibliography } \\
\text { (EUR, GLO) }\end{array}$ & $\begin{array}{l}\text { EUR: CBNM, MO, P; GLO: } \\
\text { CBNM, MO, P }\end{array}$ \\
\hline $\begin{array}{l}\text { Flacourtia indica } \\
\text { (Burm. f.) Merr. }\end{array}$ & Salicaceae & P caesp & $\begin{array}{l}\text { Africa, Madagascar, Malaysia, } \\
\text { Asia (tropical and subtropical } \\
\text { regions) }\end{array}$ & & & $x$ & & $\begin{array}{l}\text { Bibliography } \\
\text { (GLO) }\end{array}$ & GLO: CBNM, MO, P \\
\hline $\begin{array}{l}\text { Flueggea virosa } \\
\text { (Roxb. ex Willd.) } \\
\text { Royle }\end{array}$ & Phyllanthaceae & P caesp & $\begin{array}{l}\text { Southern and tropical Africa, } \\
\text { Southern Saudi Arabia, } \\
\text { Socotra, Madagascar, } \\
\text { Comoros, Mascarene islands } \\
\text { (Reunion), Pakistan to Japan } \\
\text { and Australia }\end{array}$ & & & $x$ & & $\begin{array}{l}\text { Bibliography } \\
\text { (GLO) }\end{array}$ & GLO: CBNM, MO, P \\
\hline $\begin{array}{l}\text { Furcraea foetida (L.) } \\
\text { Haw. }\end{array}$ & Asparagaceae & $\begin{array}{l}\text { Ch I } \\
\text { succ }\end{array}$ & $\begin{array}{l}\text { Central America, Greater } \\
\text { Antilles, Trinidad, South } \\
\text { America }\end{array}$ & $x$ & & $x$ & & $\begin{array}{l}\text { Bibliography } \\
\text { (EUR); Field } \\
\text { work (GLO) }\end{array}$ & \\
\hline $\begin{array}{l}\text { Furcraea selloa } \mathrm{K} . \\
\text { Koch var. marginata } \\
\text { Trel. }\end{array}$ & Asparagaceae & $\begin{array}{l}\text { Ch I } \\
\text { succ }\end{array}$ & Horticultural origin & & $x$ & & & $\begin{array}{l}\text { Field work } \\
\text { (JDN) }\end{array}$ & \\
\hline $\begin{array}{l}\text { Gossypium hirsutum } \\
\text { L. }\end{array}$ & Malvaceae & $\mathrm{P}$ caesp & $\begin{array}{l}\text { Cosmopolitan tropical; widely } \\
\text { cultivated in tropical and } \\
\text { subtropical regions }\end{array}$ & & $x$ & $x$ & & $\begin{array}{l}\text { Bibliography } \\
\text { (JDN, GLO) }\end{array}$ & $\begin{array}{l}\text { JDN: CBNM; GLO; CBNM, } \\
\text { MO, P }\end{array}$ \\
\hline
\end{tabular}




\begin{tabular}{|c|c|c|c|c|c|c|c|c|c|}
\hline \multirow[t]{2}{*}{ Scientific name } & \multirow[t]{2}{*}{ Family } & \multirow{2}{*}{$\begin{array}{l}\text { Life } \\
\text { forms }\end{array}$} & \multirow{2}{*}{$\begin{array}{l}\text { General status and } \\
\text { distribution range }\end{array}$} & \multicolumn{4}{|c|}{ Presence } & \multirow{2}{*}{$\begin{array}{l}\text { First record } \\
\text { source from } \\
\text { (name of the } \\
\text { territory) }\end{array}$} & \multirow{2}{*}{$\begin{array}{l}\text { Voucher herbarium } \\
\text { specimens (name of the } \\
\text { territory and acronym of } \\
\text { the herbarium) }\end{array}$} \\
\hline & & & & $\begin{array}{l}\text { Europ } \\
\text { a } \\
\text { (EUR) }\end{array}$ & $\begin{array}{l}\text { Juan } \\
\text { de } \\
\text { Nova } \\
\text { (JDN) }\end{array}$ & $\begin{array}{l}\text { Iles } \\
\text { Glorieuses } \\
\text { (GLO) }\end{array}$ & $\begin{array}{l}\text { Tromelin } \\
\text { (TRO) }\end{array}$ & & \\
\hline Guettarda speciosa L. & Rubiaceae & P caesp & Indo-Pacific & $x$ & $x$ & $x$ & & $\begin{array}{l}\text { Bibliography } \\
\text { (EUR, GLO); } \\
\text { Field work } \\
\text { (JDN) }\end{array}$ & $\begin{array}{l}\text { EUR: CBNM, MO, P; JDN: } \\
\text { CBNM, MO, P; GLO: } \\
\text { CBNM, MO, P }\end{array}$ \\
\hline Guilandina bonduc L. & Fabaceae & $\begin{array}{l}\mathrm{d} \text { PL / P } \\
\text { caesp }\end{array}$ & Pantropical & $x$ & $x$ & $x$ & & $\begin{array}{l}\text { Bibliography } \\
\text { (EUR, JDN, } \\
\text { GLO) }\end{array}$ & $\begin{array}{l}\text { EUR: CBNM, MO, P; JDN: } \\
\text { CBNM; GLO: CBNM, MO, } \\
\text { P }\end{array}$ \\
\hline $\begin{array}{l}\text { Gynandropsis } \\
\text { gynandra (L.) Briq. }\end{array}$ & Cleomaceae & T caesp & $\begin{array}{l}\text { Pantropical (native from } \\
\text { Africa or most widely } \\
\text { paleotropical and } \\
\text { paleosubtropical) }\end{array}$ & & & $x$ & & $\begin{array}{l}\text { Field work } \\
\text { (GLO) }\end{array}$ & GLO: CBNM, MO, P \\
\hline $\begin{array}{l}\text { Heliotropium } \\
\text { foertherianum Diane } \\
\text { \& Hilger }\end{array}$ & Boraginaceae & P caesp & $\begin{array}{l}\text { Indo-Pacific coastlines } \\
\text { (Eastern Africa and Indian } \\
\text { Ocean to Polynesia) }\end{array}$ & & $x$ & $x$ & $x$ & $\begin{array}{l}\text { Bibliography } \\
\text { (JDN, GLO, } \\
\text { TRO) }\end{array}$ & $\begin{array}{l}\text { JDN: CBNM; GLO: CBNM, } \\
\text { MO, P; TRO: CBNM, MO, } \\
\mathrm{P}\end{array}$ \\
\hline $\begin{array}{l}\text { Hernandia } \\
\text { nymphaeifolia (C. } \\
\text { Presl) Kubitzki }\end{array}$ & Hernandiaceae & P scap & Indo-Pacific coastlines & & & $x$ & & $\begin{array}{l}\text { Bibliography } \\
\text { (GLO) }\end{array}$ & GLO: CBNM, MO, P \\
\hline $\begin{array}{l}\text { Heteropogon } \\
\text { contortus (L.) P. } \\
\text { Beauv. ex Roem. \& } \\
\text { Schult. }\end{array}$ & Poaceae & H caesp & Pantropical & & $x$ & $x$ & & $\begin{array}{l}\text { Field work } \\
\text { (JDN, GLO) }\end{array}$ & $\begin{array}{l}\text { JDN: CBNM, MO, P; GLO: } \\
\text { CBNM, MO, P }\end{array}$ \\
\hline $\begin{array}{l}\text { Hibiscus physaloides } \\
\text { Guill. \& Perr. }\end{array}$ & Malvaceae & T scap & $\begin{array}{l}\text { Tropical and Southern Africa, } \\
\text { Madagascar, Comoros, } \\
\text { Seychelles }\end{array}$ & & $x$ & $x$ & & $\begin{array}{l}\text { Bibliography } \\
\text { (GLO); Field } \\
\text { work (JDN) }\end{array}$ & $\begin{array}{l}\text { JDN: CBNM, MO, P; GLO: } \\
\text { CBNM, MO, P }\end{array}$ \\
\hline
\end{tabular}




\begin{tabular}{|c|c|c|c|c|c|c|c|c|c|}
\hline \multirow[t]{2}{*}{ Scientific name } & \multirow[t]{2}{*}{ Family } & \multirow{2}{*}{$\begin{array}{l}\text { Life } \\
\text { forms }\end{array}$} & \multirow{2}{*}{$\begin{array}{l}\text { General status and } \\
\text { distribution range }\end{array}$} & \multicolumn{4}{|c|}{ Presence } & \multirow{2}{*}{$\begin{array}{l}\text { First record } \\
\text { source from } \\
\text { (name of the } \\
\text { territory) }\end{array}$} & \multirow{2}{*}{$\begin{array}{l}\text { Voucher herbarium } \\
\text { specimens (name of the } \\
\text { territory and acronym o } \\
\text { the herbarium) }\end{array}$} \\
\hline & & & & $\begin{array}{l}\text { Europ } \\
\quad \text { a } \\
\text { (EUR) }\end{array}$ & $\begin{array}{l}\text { Juan } \\
\text { de } \\
\text { Nova } \\
\text { (JDN) }\end{array}$ & $\begin{array}{l}\text { Iles } \\
\text { Glorieuses } \\
\text { (GLO) }\end{array}$ & $\begin{array}{l}\text { Tromelin } \\
\text { (TRO) }\end{array}$ & & \\
\hline Hibiscus tiliaceus L. & Malvaceae & P scap & $\begin{array}{l}\text { Tropical and subtropical } \\
\text { regions, especially in coastal } \\
\text { areas }\end{array}$ & $x$ & & $x$ & & $\begin{array}{l}\text { Field work } \\
\text { (EUR, GLO) }\end{array}$ & $\begin{array}{l}\text { EUR: CBNM, MO, P; GLO: } \\
\text { CBNM, MO, P }\end{array}$ \\
\hline $\begin{array}{l}\text { Hyphaene coriacea } \\
\text { Gaertn. }\end{array}$ & Arecaceae & P ros & $\begin{array}{l}\text { Eastern and southern Africa, } \\
\text { Madagascar, Comoros }\end{array}$ & & $x$ & & & $\begin{array}{l}\text { Bibliography } \\
\text { (JDN) }\end{array}$ & \\
\hline $\begin{array}{l}\text { Hypoestes juanensis } \\
\text { Benoist }\end{array}$ & Acanthaceae & $\begin{array}{l}\text { Ch suff } \\
\text { caesp }\end{array}$ & Juan de Nova (endemic) & & $x$ & & & $\begin{array}{l}\text { Field work } \\
\text { (JDN) }\end{array}$ & JDN: CBNM, MO, P \\
\hline $\begin{array}{l}\text { Imperata cylindrica } \\
\text { (L.) P. Beauv. }\end{array}$ & Poaceae & c G rhiz & $\begin{array}{l}\text { Paleotropical, from } \\
\text { mediterranean region to } \\
\text { South West Asia, Chili }\end{array}$ & & $x$ & $x$ & & $\begin{array}{l}\text { Field work } \\
\text { (JDN, GLO) }\end{array}$ & $\begin{array}{l}\text { JDN: CBNM, MO; GLO: } \\
\text { CBNM, MO, P }\end{array}$ \\
\hline Indigofera tinctoria L. & Fabaceae & $\begin{array}{l}\text { Ch suff } \\
\text { caesp }\end{array}$ & $\begin{array}{l}\text { Tropical Africa and tropical } \\
\text { Asia }\end{array}$ & & $x$ & & & $\begin{array}{l}\text { Field work } \\
\text { (JDN) }\end{array}$ & JDN: CBNM, MO, P \\
\hline $\begin{array}{l}\text { Ipomoea coptica (L.) } \\
\text { Roth }\end{array}$ & Convolvulaceae & $\begin{array}{l}\text { T rept / } \\
\text { d TL }\end{array}$ & $\begin{array}{l}\text { Tropical Africa and South } \\
\text { West Asia }\end{array}$ & $x$ & & & & $\begin{array}{l}\text { Field work } \\
\text { (EUR) }\end{array}$ & EUR: CBNM \\
\hline $\begin{array}{l}\text { Ipomoea pes-caprae } \\
\text { (L.) R. Br. subsp. } \\
\text { brasiliensis (L.) } \\
\text { Ooststr. }\end{array}$ & Convolvulaceae & $\begin{array}{l}\text { Ch suff } \\
\text { rept / st } \\
\text { PL }\end{array}$ & Pantropical & $x$ & $x$ & $x$ & $x$ & $\begin{array}{l}\text { Bibliography } \\
\text { (EUR, JDN, } \\
\text { GLO, TRO) }\end{array}$ & $\begin{array}{l}\text { EUR: CBNM, MO, P; JDN: } \\
\text { CBNM, MO, P; GLO: } \\
\text { CBNM, MO, P }\end{array}$ \\
\hline Ipomoea violacea $\mathrm{L}$. & Convolvulaceae & st PL & $\begin{array}{l}\text { Pantropical (except West } \\
\text { Africa), mainly on coastlines }\end{array}$ & $x$ & $x$ & $\mathrm{x}$ & & $\begin{array}{l}\text { Bibliography } \\
\text { (GLO); Field } \\
\text { work (EUR, } \\
\text { JDN) }\end{array}$ & $\begin{array}{l}\text { EUR: CBNM, MO, P; JDN: } \\
\text { CBNM; GLO: CBNM, MO, } \\
\text { P }\end{array}$ \\
\hline $\begin{array}{l}\text { Lantana } \\
\text { strigocamara R.W. } \\
\text { Sanders }\end{array}$ & Verbenaceae & P caesp & $\begin{array}{l}\text { North America, central and } \\
\text { south America, West Indies; } \\
\text { cultivated as ornemental on } \\
\text { tropical regions, naturalized } \\
\text { and invasive }\end{array}$ & & $x$ & & & $\begin{array}{l}\text { Field work } \\
\text { (JDN) }\end{array}$ & JDN: CBNM, MO, P \\
\hline
\end{tabular}




\begin{tabular}{|c|c|c|c|c|c|c|c|c|c|}
\hline \multirow[t]{2}{*}{ Scientific name } & \multirow[t]{2}{*}{ Family } & \multirow{2}{*}{$\begin{array}{l}\text { Life } \\
\text { forms }\end{array}$} & \multirow{2}{*}{$\begin{array}{l}\text { General status and } \\
\text { distribution range }\end{array}$} & \multicolumn{4}{|c|}{ Presence } & \multirow{2}{*}{$\begin{array}{l}\text { First record } \\
\text { source from } \\
\text { (name of the } \\
\text { territory) }\end{array}$} & \multirow{2}{*}{$\begin{array}{c}\text { Voucher herbarium } \\
\text { specimens (name of the } \\
\text { territory and acronym o } \\
\text { the herbarium) }\end{array}$} \\
\hline & & & & $\begin{array}{c}\text { Europ } \\
a \\
\text { (EUR) }\end{array}$ & $\begin{array}{l}\text { Juan } \\
\text { de } \\
\text { Nova } \\
\text { (JDN) }\end{array}$ & $\begin{array}{l}\text { lles } \\
\text { Glorieuses } \\
\text { (GLO) }\end{array}$ & $\begin{array}{l}\text { Tromelin } \\
\text { (TRO) }\end{array}$ & & \\
\hline $\begin{array}{l}\text { Launaea intybacea } \\
\text { (Jacq.) Beauverd }\end{array}$ & Asteraceae & T sem & $\begin{array}{l}\text { Dry regions of the New and } \\
\text { the Old tropic (islands } \\
\text { included), sometimes } \\
\text { considered native from } \\
\text { tropical America and became } \\
\text { pantropical }\end{array}$ & & $x$ & $x$ & & $\begin{array}{l}\text { Field work } \\
\text { (JDN, GLO) }\end{array}$ & $\begin{array}{l}\text { JDN: CBNM, MO, P; GLO: } \\
\text { CBNM, MO, P }\end{array}$ \\
\hline $\begin{array}{l}\text { Launaea sarmentosa } \\
\text { (Willd.) Schultz }\end{array}$ & Asteraceae & $\begin{array}{l}\text { Ch herb } \\
\text { rept }\end{array}$ & $\begin{array}{l}\text { Africa, Madagascar, } \\
\text { Seychelles, Mascarene islands } \\
\text { (Reunion and Mauritius) to } \\
\text { tropical Asia }\end{array}$ & & $x$ & $x$ & & $\begin{array}{l}\text { Bibliography } \\
\text { (GLO); Field } \\
\text { work (JDN) }\end{array}$ & $\begin{array}{l}\text { JDN: CBNM, MO, P; GLO: } \\
\text { CBNM, MO, P }\end{array}$ \\
\hline $\begin{array}{l}\text { Lepidium } \\
\text { englerianum } \\
\text { (Muschl.) Al-Shehbaz }\end{array}$ & Brassicaceae & T caesp & $\begin{array}{l}\text { Southern and Eastern Africa, } \\
\text { Madagascar }\end{array}$ & $x$ & & $x$ & $x$ & $\begin{array}{l}\text { Bibliography } \\
\text { (EUR); Field } \\
\text { work (GLO, } \\
\text { TRO) }\end{array}$ & $\begin{array}{l}\text { EUR: CBNM, MO, P; GLO: } \\
\text { CBNM, MO, P; TRO: } \\
\text { CBNM, MO, P }\end{array}$ \\
\hline $\begin{array}{l}\text { Leptadenia } \\
\text { madagascariensis } \\
\text { Decne. }\end{array}$ & Apocynaceae & st PL & Madagascar and Comoros & & $x$ & & & $\begin{array}{l}\text { Bibliography } \\
\text { (JDN) }\end{array}$ & JDN: CBNM, MO \\
\hline $\begin{array}{l}\text { Lepturus repens (G. } \\
\text { Forst.) R. Br. }\end{array}$ & Poaceae & H rept & $\begin{array}{l}\text { Coast of Southern Africa, } \\
\text { eastern tropical, Madagascar, } \\
\text { Mascarene island (Reunion } \\
\text { and Mauritius), West of the } \\
\text { Indian Ocean, Sri Lanka, } \\
\text { Malaysia, Northern Australia } \\
\text { and Polynesia }\end{array}$ & & $x$ & $x$ & & $\begin{array}{l}\text { Field work } \\
\text { (JDN, GLO) }\end{array}$ & $\begin{array}{l}\text { JDN: CBNM, MO, P; GLO: } \\
\text { CBNM, MO, P }\end{array}$ \\
\hline
\end{tabular}




\begin{tabular}{|c|c|c|c|c|c|c|c|c|c|}
\hline \multirow[t]{2}{*}{ Scientific name } & \multirow[t]{2}{*}{ Family } & \multirow{2}{*}{$\begin{array}{l}\text { Life } \\
\text { forms }\end{array}$} & \multirow{2}{*}{$\begin{array}{l}\text { General status and } \\
\text { distribution range }\end{array}$} & \multicolumn{4}{|c|}{ Presence } & \multirow{2}{*}{$\begin{array}{l}\text { First record } \\
\text { source from } \\
\text { (name of the } \\
\text { territory) }\end{array}$} & \multirow{2}{*}{$\begin{array}{l}\text { Voucher herbarium } \\
\text { specimens (name of the } \\
\text { territory and acronym of } \\
\text { the herbarium) }\end{array}$} \\
\hline & & & & $\begin{array}{l}\text { Europ } \\
\quad \text { a } \\
\text { (EUR) }\end{array}$ & $\begin{array}{l}\text { Juan } \\
\text { de } \\
\text { Nova } \\
\text { (JDN) }\end{array}$ & $\begin{array}{l}\text { lles } \\
\text { Glorieuses } \\
\text { (GLO) }\end{array}$ & $\begin{array}{l}\text { Tromelin } \\
\text { (TRO) }\end{array}$ & & \\
\hline $\begin{array}{l}\text { Leucaena } \\
\text { leucocephala (Lam.) } \\
\text { de Wit }\end{array}$ & Fabaceae & $\begin{array}{l}\text { P caesp } \\
\text { / P scap }\end{array}$ & $\begin{array}{l}\text { Supposed native from Mexico } \\
\text { and Central America; became } \\
\text { pantropical and } \\
\text { pansubtropical }\end{array}$ & & & $x$ & & $\begin{array}{l}\text { Field work } \\
\text { (GLO) }\end{array}$ & GLO: CBNM, MO, P \\
\hline $\begin{array}{l}\text { Lumnitzera racemosa } \\
\text { Willd. }\end{array}$ & Combretaceae & P scap & Indo-Pacific coastlines & & $x$ & & & $\begin{array}{l}\text { Field work } \\
\text { (JDN) }\end{array}$ & JDN: CBNM, MO, P \\
\hline $\begin{array}{l}\text { Lycium elliotii } \\
\text { Dammer s. I. }\end{array}$ & Solanaceae & $\begin{array}{l}\text { Ch frut } \\
\text { caesp / } \\
\text { P caesp } \\
\text { / Ch I } \\
\text { succ }\end{array}$ & $\begin{array}{l}\text { Mascarene islands, South and } \\
\text { South-East Africa, } \\
\text { Madagascar }\end{array}$ & $x$ & & & & $\begin{array}{l}\text { Field work } \\
\text { (EUR) }\end{array}$ & EUR: CBNM, MO, P \\
\hline $\begin{array}{l}\text { Lygodium kerstenii } \\
\text { Kuhn }\end{array}$ & Lygodiaceae & $\begin{array}{l}\text { Ch herb } \\
\text { rept }\end{array}$ & $\begin{array}{l}\text { East Africa, Madagascar, } \\
\text { Comoros }\end{array}$ & & & $x$ & & $\begin{array}{l}\text { Field work } \\
\text { (GLO) }\end{array}$ & GLO: CBNM, MO, P \\
\hline $\begin{array}{l}\text { Maerua baillonii } \\
\text { Hadj-Moust. }\end{array}$ & Capparaceae & P caesp & Madagascar & & $x$ & & & $\begin{array}{l}\text { Field work } \\
\text { (JDN) }\end{array}$ & JDN: CBNM, MO, P \\
\hline $\begin{array}{l}\text { Malvastrum } \\
\text { coromandelianum } \\
\text { (L.) Garcke }\end{array}$ & Malvaceae & $\begin{array}{l}\text { Ch suff } \\
\text { caesp / } \\
\text { T caesp }\end{array}$ & $\begin{array}{l}\text { Central and South America; } \\
\text { became pantropical }\end{array}$ & $x$ & & $x$ & & $\begin{array}{l}\text { Bibliography } \\
\text { (GLO); Field } \\
\text { work (EUR) }\end{array}$ & $\begin{array}{l}\text { EUR: CBNM, MO, P; GLO: } \\
\text { CBNM, MO, P }\end{array}$ \\
\hline $\begin{array}{l}\text { Manihot esculenta } \\
\text { Crantz }\end{array}$ & Euphorbiaceae & P caesp & $\begin{array}{l}\text { South America; widely } \\
\text { cultivated in tropical regions }\end{array}$ & & $x$ & & & $\begin{array}{l}\text { Bibliography } \\
\text { (JDN) }\end{array}$ & \\
\hline $\begin{array}{l}\text { Marsdenia verrucosa } \\
\text { Decne. }\end{array}$ & Apocynaceae & P caesp & Madagascar & & $x$ & & & $\begin{array}{l}\text { Field work } \\
\text { (JDN) }\end{array}$ & JDN: CBNM \\
\hline $\begin{array}{l}\text { Mimusops caffra E. } \\
\text { Mey. ex A. DC. }\end{array}$ & Sapotaceae & P scap & $\begin{array}{l}\text { South East and Southern } \\
\text { african coastlines, from } \\
\text { Mozambique to the East of } \\
\text { The Cap }\end{array}$ & $x$ & & & & $\begin{array}{l}\text { Field work } \\
\text { (EUR) }\end{array}$ & \\
\hline
\end{tabular}




\begin{tabular}{|c|c|c|c|c|c|c|c|c|c|}
\hline \multirow[t]{2}{*}{ Scientific name } & \multirow[t]{2}{*}{ Family } & \multirow{2}{*}{$\begin{array}{l}\text { Life } \\
\text { forms }\end{array}$} & \multirow{2}{*}{$\begin{array}{l}\text { General status and } \\
\text { distribution range }\end{array}$} & \multicolumn{4}{|c|}{ Presence } & \multirow{2}{*}{$\begin{array}{l}\text { First record } \\
\text { source from } \\
\text { (name of the } \\
\text { territory) }\end{array}$} & \multirow{2}{*}{$\begin{array}{l}\text { Voucher herbarium } \\
\text { specimens (name of the } \\
\text { territory and acronym of } \\
\text { the herbarium) }\end{array}$} \\
\hline & & & & $\begin{array}{l}\text { Europ } \\
\text { a } \\
\text { (EUR) }\end{array}$ & $\begin{array}{l}\text { Juan } \\
\text { de } \\
\text { Nova } \\
\text { (JDN) }\end{array}$ & $\begin{array}{c}\text { lles } \\
\text { Glorieuses } \\
\text { (GLO) }\end{array}$ & $\begin{array}{l}\text { Tromelin } \\
\text { (TRO) }\end{array}$ & & \\
\hline $\begin{array}{l}\text { Mimusops coriacea } \\
\text { (A. DC.) Miq. }\end{array}$ & Sapotaceae & P scap & $\begin{array}{l}\text { Madagascar, Comoros } \\
\text { (Anjouan and Mayotte); } \\
\text { introduced and naturalized } \\
\text { elsewhere in tropical regions }\end{array}$ & & $x$ & & & $\begin{array}{l}\text { Field work } \\
\text { (JDN) }\end{array}$ & JDN: CBNM, MO, P \\
\hline $\begin{array}{l}\text { Mollugo nudicaulis } \\
\text { Lam. }\end{array}$ & Molluginaceae & Tros & Pantropical & $x$ & $x$ & $x$ & & $\begin{array}{l}\text { Bibliography } \\
\text { (EUR); Field } \\
\text { work (JDN, } \\
\text { GLO) }\end{array}$ & $\begin{array}{l}\text { EUR: CBNM, MO, P; JDN: } \\
\text { CBNM; GLO: CBNM, MO }\end{array}$ \\
\hline Morinda citrifolia $L$. & Rubiaceae & P caesp & $\begin{array}{l}\text { India and Ceylan to Malaysia, } \\
\text { Northern Australia, Pacific }\end{array}$ & & & $x$ & & $\begin{array}{l}\text { Field work } \\
\text { (GLO) }\end{array}$ & GLO : CBNM, MO, P \\
\hline $\begin{array}{l}\text { Moringa oleifera } \\
\text { Lam. }\end{array}$ & Moringaceae & P scap & $\begin{array}{l}\text { Probably native from the } \\
\text { North West of India and } \\
\text { Pakistan; introduced and } \\
\text { cultivated in tropical and } \\
\text { subtropical regions, } \\
\text { sometimes naturalized }\end{array}$ & $x$ & $x$ & $x$ & & $\begin{array}{l}\text { Bibliography } \\
\text { (EUR); Field } \\
\text { work (JDN, } \\
\text { GLO) }\end{array}$ & $\begin{array}{l}\text { EUR: CBNM, MO, P; GLO: } \\
\text { CBNM, MO, P }\end{array}$ \\
\hline Morus alba L. & Moraceae & P scap & $\begin{array}{l}\text { Central and Eastern China; } \\
\text { widely cultivated in } \\
\text { temperates regions and often } \\
\text { naturalized (South and } \\
\text { tropical Africca, America) }\end{array}$ & & $x$ & & & $\begin{array}{l}\text { Field work } \\
\text { (JDN) }\end{array}$ & \\
\hline $\begin{array}{l}\text { Mucuna gigantea } \\
\text { (Willd.) DC. }\end{array}$ & Fabaceae & st PL & $\begin{array}{l}\text { Tropical Africa to French } \\
\text { Polynesia }\end{array}$ & & $x$ & $x$ & & $\begin{array}{l}\text { Field work } \\
\text { (JDN, GLO) }\end{array}$ & GLO : CBNM, MO, P \\
\hline
\end{tabular}




\begin{tabular}{|c|c|c|c|c|c|c|c|c|c|}
\hline \multirow[t]{2}{*}{ Scientific name } & \multirow[t]{2}{*}{ Family } & \multirow{2}{*}{$\begin{array}{l}\text { Life } \\
\text { forms }\end{array}$} & \multirow{2}{*}{$\begin{array}{l}\text { General status and } \\
\text { distribution range }\end{array}$} & \multicolumn{4}{|c|}{ Presence } & \multirow{2}{*}{$\begin{array}{l}\text { First record } \\
\text { source from } \\
\text { (name of the } \\
\text { territory) }\end{array}$} & \multirow{2}{*}{$\begin{array}{l}\text { Voucher herbarium } \\
\text { specimens (name of the } \\
\text { territory and acronym of } \\
\text { the herbarium) }\end{array}$} \\
\hline & & & & $\begin{array}{c}\text { Europ } \\
a \\
\text { (EUR) }\end{array}$ & $\begin{array}{l}\text { Juan } \\
\text { de } \\
\text { Nova } \\
\text { (JDN) }\end{array}$ & $\begin{array}{c}\text { Iles } \\
\text { Glorieuses } \\
\text { (GLO) }\end{array}$ & $\begin{array}{l}\text { Tromelin } \\
\text { (TRO) }\end{array}$ & & \\
\hline Musa sp. & Musaceae & $P$ herb & ? & & $x$ & $x$ & $x$ & $\begin{array}{l}\text { Bibliography } \\
\text { (JDN, GLO, } \\
\text { TRO) }\end{array}$ & \\
\hline $\begin{array}{l}\text { Nephrolepis biserrata } \\
\text { (Sw.) Schott }\end{array}$ & $\begin{array}{l}\text { Nephrolepidacea } \\
\text { e }\end{array}$ & $\begin{array}{l}\text { Ch herb } \\
\text { rept }\end{array}$ & Pantropical & & & $x$ & & $\begin{array}{l}\text { Field work } \\
\text { (GLO) }\end{array}$ & GLO : CBNM, MO, P \\
\hline $\begin{array}{l}\text { Nervilia bicarinata } \\
\text { (Blume) Schltr. }\end{array}$ & Orchidaceae & c G bulb & $\begin{array}{l}\text { Tropical Africa, Eastern Africa, } \\
\text { Saudi Arabia, Madagascar, } \\
\text { Comoros (Grande Comore, } \\
\text { Mayotte), Mascarene islands } \\
\text { (Reunion and Mauritius) }\end{array}$ & & $x$ & $x$ & & $\begin{array}{l}\text { Field work } \\
\text { (JDN, GLO) }\end{array}$ & $\begin{array}{l}\text { JDN: CBNM; GLO: CBNM, } \\
\text { MO, P }\end{array}$ \\
\hline $\begin{array}{l}\text { Nesogenes } \\
\text { madagascariensis } \\
\text { (Bonati) Marais }\end{array}$ & Orobanchaceae & T caesp & Madagascar & & $x$ & & & $\begin{array}{l}\text { Field work } \\
\text { (JDN) }\end{array}$ & JDN: CBNM, MO, P \\
\hline $\begin{array}{l}\text { Nesogenes prostrata } \\
\text { (Benth.) Hemsl. }\end{array}$ & Orobanchaceae & Trept & Coral Seychelles & & & $x$ & & $\begin{array}{l}\text { Field work } \\
\text { (GLO) }\end{array}$ & GLO: CBNM, MO, P \\
\hline $\begin{array}{l}\text { Ochrosia } \\
\text { oppositifolia (Lam.) } \\
\text { K. Schum. }\end{array}$ & Apocynaceae & P scap & $\begin{array}{l}\text { Indo-Pacific (Seychelles to } \\
\text { Polynesia) }\end{array}$ & & & $x$ & & $\begin{array}{l}\text { Bibliography } \\
\text { (GLO) }\end{array}$ & GLO: CBNM, MO, P \\
\hline $\begin{array}{l}\text { Oldenlandia } \\
\text { corymbosa L. var. } \\
\text { caespitosa (Benth.) } \\
\text { Verdc. }\end{array}$ & Rubiaceae & T caesp & Tropical Africa & $x$ & $x$ & & & $\begin{array}{l}\text { Field work } \\
\text { (EUR, JDN) }\end{array}$ & $\begin{array}{l}\text { EUR: CBNM, MO, P; JDN: } \\
\text { CBNM, MO, P }\end{array}$ \\
\hline
\end{tabular}




\begin{tabular}{|c|c|c|c|c|c|c|c|c|c|}
\hline \multirow[t]{2}{*}{ Scientific name } & \multirow[t]{2}{*}{ Family } & \multirow{2}{*}{$\begin{array}{l}\text { Life } \\
\text { forms }\end{array}$} & \multirow{2}{*}{$\begin{array}{l}\text { General status and } \\
\text { distribution range }\end{array}$} & \multicolumn{4}{|c|}{ Presence } & \multirow{2}{*}{$\begin{array}{l}\text { First record } \\
\text { source from } \\
\text { (name of the } \\
\text { territory) }\end{array}$} & \multirow{2}{*}{$\begin{array}{c}\text { Voucher herbarium } \\
\text { specimens (name of the } \\
\text { territory and acronym o } \\
\text { the herbarium) }\end{array}$} \\
\hline & & & & $\begin{array}{l}\text { Europ } \\
\quad a \\
\text { (EUR) }\end{array}$ & $\begin{array}{l}\text { Juan } \\
\text { de } \\
\text { Nova } \\
\text { (JDN) }\end{array}$ & $\begin{array}{l}\text { lles } \\
\text { Glorieuses } \\
\text { (GLO) }\end{array}$ & $\begin{array}{l}\text { Tromelin } \\
\text { (TRO) }\end{array}$ & & \\
\hline $\begin{array}{l}\text { Oldenlandia } \\
\text { corymbosa L. var. } \\
\text { corymbosa }\end{array}$ & Rubiaceae & T scap & $\begin{array}{l}\text { Native from Africa and India; } \\
\text { now widespread throughout } \\
\text { tropical and subtropical } \\
\text { regions }\end{array}$ & $x$ & & $x$ & & $\begin{array}{l}\text { Field work } \\
\text { (EUR, GLO) }\end{array}$ & $\begin{array}{l}\text { EUR: CBNM, MO, P; GLO: } \\
\text { CBNM, MO, P }\end{array}$ \\
\hline $\begin{array}{l}\text { Oldenlandia } \\
\text { fastigiata Bremek. } \\
\text { var. fastigiata }\end{array}$ & Rubiaceae & T scap & Africa, Madagascar & & $x$ & & & $\begin{array}{l}\text { Field work } \\
\text { (JDN) }\end{array}$ & JDN: CBNM, MO, P \\
\hline $\begin{array}{l}\text { Oldenlandia } \\
\text { fastigiata Bremek. } \\
\text { var. somala } \\
\text { (Bremek.) Verdc. }\end{array}$ & Rubiaceae & T scap & Africa & & $x$ & & & $\begin{array}{l}\text { Field work } \\
\text { (JDN) }\end{array}$ & JDN: CBNM, MO, P \\
\hline $\begin{array}{l}\text { Ophioglossum } \\
\text { lancifolium C. Presl }\end{array}$ & Ophioglossaceae & c G rhiz & $\begin{array}{l}\text { Southern and Eastern Africa, } \\
\text { Congo, Madagascar, Comoros, } \\
\text { Mascarene islands (Reunion } \\
\text { and Mauritius) }\end{array}$ & $x$ & $x$ & & & $\begin{array}{l}\text { Field work } \\
\text { (EUR, JDN) }\end{array}$ & $\begin{array}{l}\text { EUR: CBNM, MO, P; JDN: } \\
\text { CBNM, MO }\end{array}$ \\
\hline $\begin{array}{l}\text { Ophioglossum } \\
\text { polyphyllum A. Braun }\end{array}$ & Ophioglossaceae & c G rhiz & $\begin{array}{l}\text { Africa, India, Hawai, Central } \\
\text { America }\end{array}$ & $x$ & & & & $\begin{array}{l}\text { Field work } \\
\text { (EUR) }\end{array}$ & EUR: CBNM, MO, P \\
\hline $\begin{array}{l}\text { Opuntia stricta } \\
\text { (Haw.) Haw. }\end{array}$ & Cactaceae & $P$ succ & $\begin{array}{l}\text { North America, India, China, } \\
\text { Africa, Madagascar }\end{array}$ & & $x$ & & & $\begin{array}{l}\text { Field work } \\
\text { (JDN) }\end{array}$ & \\
\hline Pandanus utilis Bory & Pandanaceae & P scap & $\begin{array}{l}\text { Supposed native from } \\
\text { Mascarene islands; } \\
\text { introduced elsewhere }\end{array}$ & & & & $x$ & $\begin{array}{l}\text { Bibliography } \\
\text { (TRO) }\end{array}$ & \\
\hline $\begin{array}{l}\text { Panicum } \\
\text { pseudowoeltzkowii A. } \\
\text { Camus }\end{array}$ & Poaceae & H rept & $\begin{array}{l}\text { Madagascar; also present in } \\
\text { Mascarene islands (Reunion), } \\
\text { but indigeneous status } \\
\text { doubtful }\end{array}$ & $x$ & & & & $\begin{array}{l}\text { Field work } \\
\text { (EUR) }\end{array}$ & EUR: CBNM, MO, P \\
\hline
\end{tabular}




\begin{tabular}{|c|c|c|c|c|c|c|c|c|c|}
\hline \multirow[t]{2}{*}{ Scientific name } & \multirow[t]{2}{*}{ Family } & \multirow{2}{*}{$\begin{array}{l}\text { Life } \\
\text { forms }\end{array}$} & \multirow{2}{*}{$\begin{array}{l}\text { General status and } \\
\text { distribution range }\end{array}$} & \multicolumn{4}{|c|}{ Presence } & \multirow{2}{*}{$\begin{array}{l}\text { First record } \\
\text { source from } \\
\text { (name of the } \\
\text { territory) }\end{array}$} & \multirow{2}{*}{$\begin{array}{c}\text { Voucher herbarium } \\
\text { specimens (name of the } \\
\text { territory and acronym o } \\
\text { the herbarium) }\end{array}$} \\
\hline & & & & $\begin{array}{c}\text { Europ } \\
a \\
\text { (EUR) }\end{array}$ & $\begin{array}{l}\text { Juan } \\
\text { de } \\
\text { Nova } \\
\text { (JDN) }\end{array}$ & $\begin{array}{l}\text { Iles } \\
\text { Glorieuses } \\
\text { (GLO) }\end{array}$ & $\begin{array}{l}\text { Tromelin } \\
\text { (TRO) }\end{array}$ & & \\
\hline $\begin{array}{l}\text { Panicum voeltzkowii } \\
\text { Mez s. I. }\end{array}$ & Poaceae & $\begin{array}{l}\text { H caesp } \\
\text { / Ch } \\
\text { herb } \\
\text { caesp }\end{array}$ & Madagascar, coral Seychelles & $x$ & $x$ & $x$ & & $\begin{array}{l}\text { Field work } \\
\text { (EUR, JDN, } \\
\text { GLO) }\end{array}$ & $\begin{array}{l}\text { EUR: CBNM, MO, P; JDN: } \\
\text { CBNM, MO, P; GLO: } \\
\text { CBNM, MO, P }\end{array}$ \\
\hline $\begin{array}{l}\text { Paspalum vaginatum } \\
\text { Sw. }\end{array}$ & Poaceae & $\begin{array}{l}\text { Ch herb } \\
\text { rept }\end{array}$ & $\begin{array}{l}\text { Tropical and warm temperate } \\
\text { regions }\end{array}$ & & & $x$ & & $\begin{array}{l}\text { Field work } \\
\text { (GLO) }\end{array}$ & GLO: CBNM, MO, P \\
\hline Passiflora suberosa L. & Passifloraceae & el PL & $\begin{array}{l}\text { Tropical America; naturalized } \\
\text { elsewhere in tropical regions }\end{array}$ & & & $x$ & & $\begin{array}{l}\text { Bibliography } \\
\text { (GLO) }\end{array}$ & GLO: CBNM, MO, P \\
\hline $\begin{array}{l}\text { Pemphis acidula J.R. } \\
\text { Forst. \& G. Forst. }\end{array}$ & Lythraceae & P caesp & Indo-Pacific & $x$ & $x$ & $x$ & & $\begin{array}{l}\text { Bibliography } \\
\text { (EUR, JDN, } \\
\text { GLO) }\end{array}$ & $\begin{array}{l}\text { EUR: CBNM, MO, P; JDN: } \\
\text { CBNM, MO; GLO: CBNM, } \\
\text { MO, P }\end{array}$ \\
\hline $\begin{array}{l}\text { Perrierophytum } \\
\text { glomeratum Hochr. }\end{array}$ & Malvaceae & P caesp & $\begin{array}{l}\text { Iles Eparses (Juan de Nova, } \\
\text { Grande Glorieuse) }\end{array}$ & & $x$ & $x$ & & $\begin{array}{l}\text { Bibliography } \\
\text { (JDN); Field } \\
\text { work (GLO) }\end{array}$ & $\begin{array}{l}\text { JDN: CBNM, MO, P; GLO: } \\
\text { CBNM, MO, P }\end{array}$ \\
\hline $\begin{array}{l}\text { Persea americana } \\
\text { Mill. }\end{array}$ & Lauraceae & P scap & $\begin{array}{l}\text { Supposed native from Central } \\
\text { America }\end{array}$ & & & & $\mathrm{X}$ & $\begin{array}{l}\text { Bibliography } \\
\text { (TRO) }\end{array}$ & \\
\hline Phoenix dactylifera L. & Arecaceae & P ros & $\begin{array}{l}\text { Supposed native from North } \\
\text { East Sahara and Saudi Arabia } \\
\text { (form with small inedible } \\
\text { fruit); edible form of } \\
\text { horticultural origin probably in } \\
\text { Middle East }\end{array}$ & $x$ & & & & $\begin{array}{l}\text { Bibliography } \\
\text { (EUR) }\end{array}$ & \\
\hline
\end{tabular}




\begin{tabular}{|c|c|c|c|c|c|c|c|c|c|}
\hline \multirow[t]{2}{*}{ Scientific name } & \multirow[t]{2}{*}{ Family } & \multirow{2}{*}{$\begin{array}{l}\text { Life } \\
\text { forms }\end{array}$} & \multirow{2}{*}{$\begin{array}{l}\text { General status and } \\
\text { distribution range }\end{array}$} & \multicolumn{4}{|c|}{ Presence } & \multirow{2}{*}{$\begin{array}{l}\text { First record } \\
\text { source from } \\
\text { (name of the } \\
\text { territory) }\end{array}$} & \multirow{2}{*}{$\begin{array}{l}\text { Voucher herbarium } \\
\text { specimens (name of the } \\
\text { territory and acronym of } \\
\text { the herbarium) }\end{array}$} \\
\hline & & & & $\begin{array}{c}\text { Europ } \\
a \\
\text { (EUR) }\end{array}$ & $\begin{array}{l}\text { Juan } \\
\text { de } \\
\text { Nova } \\
\text { (JDN) }\end{array}$ & $\begin{array}{l}\text { Iles } \\
\text { Glorieuses } \\
\text { (GLO) }\end{array}$ & $\begin{array}{l}\text { Tromelin } \\
\text { (TRO) }\end{array}$ & & \\
\hline $\begin{array}{l}\text { Phyllanthus amarus } \\
\text { Schumach. }\end{array}$ & Phyllanthaceae & T scap & $\begin{array}{l}\text { Supposed native from tropical } \\
\text { America; became pantropical }\end{array}$ & & $x$ & $x$ & & $\begin{array}{l}\text { Bibliography } \\
\text { (GLO); Field } \\
\text { work (JDN) }\end{array}$ & GLO: CBNM, MO, P \\
\hline $\begin{array}{l}\text { Phyllanthus } \\
\text { coluteoides Baill. ex } \\
\text { Müll.Arg. }\end{array}$ & Phyllanthaceae & P caesp & Madagascar & & $x$ & & & $\begin{array}{l}\text { Bibliography } \\
\text { (JDN) }\end{array}$ & JDN: CBNM \\
\hline $\begin{array}{l}\text { Phyllanthus } \\
\text { maderaspatensis L. s. } \\
\text { I. }\end{array}$ & Phyllanthaceae & T caesp & $\begin{array}{l}\text { India, West of the Indian } \\
\text { Ocean }\end{array}$ & $x$ & $x$ & $x$ & & $\begin{array}{l}\text { Bibliography } \\
\text { (EUR, GLO); } \\
\text { Field work } \\
\text { (JDN) }\end{array}$ & $\begin{array}{l}\text { EUR: CBNM, MO, P; JDN: } \\
\text { CBNM, MO, P; GLO: } \\
\text { CBNM, MO, P }\end{array}$ \\
\hline Phyllanthus sp.1 & Phyllanthaceae & T caesp & $?$ & $x$ & & & & $\begin{array}{l}\text { Field work } \\
\text { (EUR) }\end{array}$ & EUR: CBNM, MO, P \\
\hline Phyllanthus sp.2 & Phyllanthaceae & T scap & ? & $x$ & & & & $\begin{array}{l}\text { Field work } \\
\text { (EUR) }\end{array}$ & EUR: CBNM, MO, P \\
\hline $\begin{array}{l}\text { Phymatosorus } \\
\text { scolopendria (Burm. } \\
\text { f.) Pic. Serm. }\end{array}$ & Polypodiaceae & $\begin{array}{l}\text { Ch herb } \\
\text { rept }\end{array}$ & Tropical Africa and Asia & & & $x$ & & $\begin{array}{l}\text { Field work } \\
\text { (GLO) }\end{array}$ & GLO: CBNM, MO, P \\
\hline $\begin{array}{l}\text { Physalis lagascae } \\
\text { Roem. \& Schult. }\end{array}$ & Solanaceae & T caesp & $\begin{array}{l}\text { Native to tropical America; } \\
\text { now almost cosmopolitan, } \\
\text { widely naturalized in the } \\
\text { tropical and subtropical } \\
\text { regions in Africa and Asia }\end{array}$ & & $x$ & $\mathrm{X}$ & & $\begin{array}{l}\text { Field work } \\
\text { (JDN, GLO) }\end{array}$ & $\begin{array}{l}\text { JDN: CBNM, MO, P; GLO: } \\
\text { CBNM, MO, P }\end{array}$ \\
\hline Pisonia grandis $\mathrm{R}$. Br. & Nyctaginaceae & P scap & Pacific to Madagascar & $x$ & $x$ & $x$ & & $\begin{array}{l}\text { Bibliography } \\
\text { (EUR, GLO); } \\
\text { Field work } \\
\text { (JDN) }\end{array}$ & $\begin{array}{l}\text { EUR: CBNM, MO, P; JDN: } \\
\text { CBNM; GLO: CBNM, MO, } \\
\text { P }\end{array}$ \\
\hline $\begin{array}{l}\text { Pithecellobium dulce } \\
\text { (Roxb.) Benth. }\end{array}$ & Fabaceae & P scap & Central America & & $x$ & & & $\begin{array}{l}\text { Field work } \\
\text { (JDN) }\end{array}$ & JDN: CBNM, MO \\
\hline
\end{tabular}




\begin{tabular}{|c|c|c|c|c|c|c|c|c|c|}
\hline \multirow[t]{2}{*}{ Scientific name } & \multirow[t]{2}{*}{ Family } & \multirow{2}{*}{$\begin{array}{l}\text { Life } \\
\text { forms }\end{array}$} & \multirow{2}{*}{$\begin{array}{l}\text { General status and } \\
\text { distribution range }\end{array}$} & \multicolumn{4}{|c|}{ Presence } & \multirow{2}{*}{$\begin{array}{l}\text { First record } \\
\text { source from } \\
\text { (name of the } \\
\text { territory) }\end{array}$} & \multirow{2}{*}{$\begin{array}{l}\text { Voucher herbarium } \\
\text { specimens (name of the } \\
\text { territory and acronym of } \\
\text { the herbarium) }\end{array}$} \\
\hline & & & & $\begin{array}{l}\text { Europ } \\
\text { a } \\
\text { (EUR) }\end{array}$ & $\begin{array}{l}\text { Juan } \\
\text { de } \\
\text { Nova } \\
\text { (JDN) }\end{array}$ & $\begin{array}{l}\text { lles } \\
\text { Glorieuses } \\
\text { (GLO) }\end{array}$ & $\begin{array}{l}\text { Tromelin } \\
\text { (TRO) }\end{array}$ & & \\
\hline $\begin{array}{l}\text { Pleurostelma } \\
\text { cernuum (Decne.) } \\
\text { Bullock }\end{array}$ & Apocynaceae & st PL & Eastern Africa, Comoros & & $x$ & & & $\begin{array}{l}\text { Field work } \\
\text { (JDN) }\end{array}$ & JDN: CBNM, MO, P \\
\hline $\begin{array}{l}\text { Plumbago aphylla } \\
\text { Boj. ex Boiss. }\end{array}$ & Plumbaginaceae & $\begin{array}{l}\text { Ch herb } \\
\text { caesp }\end{array}$ & $\begin{array}{l}\text { Eastern Africa, West of the } \\
\text { Indian Ocean }\end{array}$ & $x$ & & & & $\begin{array}{l}\text { Bibliography } \\
\text { (EUR) }\end{array}$ & EUR: CBNM, MO, P \\
\hline $\begin{array}{l}\text { Plumeria rubra L. f. } \\
\text { tricolor (Ruiz \& Pav.) } \\
\text { Woodson }\end{array}$ & Apocynaceae & $\mathrm{P}$ caesp & $\begin{array}{l}\text { Unknown origin; widely } \\
\text { cultivated in tropical regions }\end{array}$ & & $x$ & & & $\begin{array}{l}\text { Field work } \\
\text { (JDN) }\end{array}$ & \\
\hline $\begin{array}{l}\text { Pongamia pinnata } \\
\text { (L.) Pierre }\end{array}$ & Fabaceae & P scap & $\begin{array}{l}\text { Tropical Asia, Australia, Pacific } \\
\text { islands }\end{array}$ & & $x$ & & & $\begin{array}{l}\text { Field work } \\
\text { (JDN) }\end{array}$ & \\
\hline $\begin{array}{l}\text { Portulaca } \\
\text { granulatostellulata } \\
\text { (Poelln.) Ricceri \& } \\
\text { Arrigoni }\end{array}$ & Portulacaceae & T succ & $\begin{array}{l}\text { Seychelles, East Africa, } \\
\text { Mascarene islands, Europe, } \\
\text { Asia, Egypt (world wide } \\
\text { distribution) }\end{array}$ & $x$ & $x$ & $x$ & & $\begin{array}{l}\text { Bibliography } \\
\text { (EUR, JDN, } \\
\text { GLO) }\end{array}$ & $\begin{array}{l}\text { EUR: CBNM; JDN: CBNM; } \\
\text { GLO: CBNM, MO }\end{array}$ \\
\hline $\begin{array}{l}\text { Portulaca } \\
\text { mauritiensis Poelln. } \\
\text { var. aldabrensis } \\
\text { Fosberg }\end{array}$ & Portulacaceae & T succ & $\begin{array}{l}\text { Endemic Seychelles (Aldabra, } \\
\text { Assumption, Cosmoledo, } \\
\text { Farquhar) }\end{array}$ & & & $x$ & $x$ & $\begin{array}{l}\text { Bibliography } \\
\text { (GLO); Field } \\
\text { work (TRO) }\end{array}$ & $\begin{array}{l}\text { GLO: CBNM, MO, P; TRO: } \\
\text { CBNM, MO, P }\end{array}$ \\
\hline $\begin{array}{l}\text { Portulaca nitida } \\
\text { (Danin et H.G. Baker) } \\
\text { Ricceri et Arrigoni }\end{array}$ & Portulacaceae & T succ & $\begin{array}{l}\text { Aldabara Island, Ascension } \\
\text { Island, Azores, Egypt, England, } \\
\text { France, Israel, Tanzania, USA }\end{array}$ & $x$ & $x$ & $x$ & & $\begin{array}{l}\text { Field work } \\
\text { (EUR, JDN, } \\
\text { GLO) }\end{array}$ & $\begin{array}{l}\text { EUR: CBNM; JDN: CBNM; } \\
\text { GLO: CBNM }\end{array}$ \\
\hline $\begin{array}{l}\text { Portulaca gr. } \\
\text { oleracea } \mathrm{L} .\end{array}$ & Portulacaceae & T succ & Cosmopolitan & & & & $x$ & $\begin{array}{l}\text { Bibliography } \\
\text { (TRO) }\end{array}$ & TRO: CBNM, MO, P \\
\hline $\begin{array}{l}\text { Portulaca aff. } \\
\text { tuberosa Roxb. }\end{array}$ & Portulacaceae & T succ & $\begin{array}{l}\text { Maldives, Sri Lanka, Pakistan, } \\
\text { India, Australia, Pacific islands }\end{array}$ & $x$ & & & & $\begin{array}{l}\text { Bibliography } \\
\text { (EUR) }\end{array}$ & EUR: CBNM \\
\hline Premna serratifolia L. & Lamiaceae & $\begin{array}{l}P \text { caesp } \\
/ \text { d PL }\end{array}$ & Indo-Pacific & & & $x$ & & $\begin{array}{l}\text { Field work } \\
\text { (GLO) }\end{array}$ & GLO: CBNM, MO, P \\
\hline
\end{tabular}




\begin{tabular}{|c|c|c|c|c|c|c|c|c|c|}
\hline \multirow[t]{2}{*}{ Scientific name } & \multirow[t]{2}{*}{ Family } & \multirow{2}{*}{$\begin{array}{l}\text { Life } \\
\text { forms }\end{array}$} & \multirow{2}{*}{$\begin{array}{l}\text { General status and } \\
\text { distribution range }\end{array}$} & \multicolumn{4}{|c|}{ Presence } & \multirow{2}{*}{$\begin{array}{c}\text { First record } \\
\text { source from } \\
\text { (name of the } \\
\text { territory) }\end{array}$} & \multirow{2}{*}{$\begin{array}{c}\text { Voucher herbarium } \\
\text { specimens (name of the } \\
\text { territory and acronym o } \\
\text { the herbarium) }\end{array}$} \\
\hline & & & & $\begin{array}{l}\text { Europ } \\
\quad \text { a } \\
\text { (EUR) }\end{array}$ & $\begin{array}{l}\text { Juan } \\
\text { de } \\
\text { Nova } \\
\text { (JDN) }\end{array}$ & $\begin{array}{l}\text { Iles } \\
\text { Glorieuses } \\
\text { (GLO) }\end{array}$ & $\begin{array}{l}\text { Tromelin } \\
\text { (TRO) }\end{array}$ & & \\
\hline $\begin{array}{l}\text { Pseuderanthemum } \\
\text { carruthersii (Seem.) } \\
\text { Guill. var. } \\
\text { atropurpureum (W. } \\
\text { Bull) Fosberg }\end{array}$ & Acanthaceae & P caesp & $\begin{array}{l}\text { Supposed native from New } \\
\text { Hebrides; widely cultivated in } \\
\text { tropical regions }\end{array}$ & & $x$ & & & $\begin{array}{l}\text { Field work } \\
\text { (JDN) }\end{array}$ & JDN: CBNM, MO, P \\
\hline $\begin{array}{l}\text { Pseudoconyza } \\
\text { viscosa (Mill.) D'Arcy }\end{array}$ & Asteraceae & T scap & Tropical Africa and Asia & & $x$ & $x$ & & $\begin{array}{l}\text { Field work } \\
\text { (JDN, GLO) }\end{array}$ & $\begin{array}{l}\text { JDN: CBNM, MO; GLO: } \\
\text { CBNM, MO, P }\end{array}$ \\
\hline $\begin{array}{l}\text { Psiadia altissima } \\
\text { (DC.) Drake }\end{array}$ & Asteraceae & P caesp & Madagascar & $x$ & $x$ & & & $\begin{array}{l}\text { Bibliography } \\
\text { (EUR, JDN) }\end{array}$ & $\begin{array}{l}\text { EUR: CBNM, MO, P; JDN: } \\
\text { CBNM, MO, P }\end{array}$ \\
\hline $\begin{array}{l}\text { Psilotum nudum (L.) } \\
\text { P. Beauv. }\end{array}$ & Psilotaceae & $\begin{array}{l}\text { Ch herb } \\
\text { caesp }\end{array}$ & $\begin{array}{l}\text { Tropical and subtropical } \\
\text { regions }\end{array}$ & & & $x$ & & $\begin{array}{l}\text { Field work } \\
\text { (GLO) }\end{array}$ & GLO: CBNM, MO, P \\
\hline Pteris linearis Poir. & Pteridaceae & $\begin{array}{l}\text { Ch herb } \\
\text { caesp }\end{array}$ & $\begin{array}{l}\text { Tropical Africa, Madagascar, } \\
\text { Comoros, tropical Asia } \\
\text { Mascarene islands (Reunion } \\
\text { and Mauritius) }\end{array}$ & & & $x$ & & $\begin{array}{l}\text { Field work } \\
\text { (GLO) }\end{array}$ & GLO: CBNM, MO \\
\hline $\begin{array}{l}\text { Rhizophora } \\
\text { mucronata Lam. }\end{array}$ & Rhizophoraceae & P scap & $\begin{array}{l}\text { Indo-Pacific coastlines } \\
\text { (Eastern Africac to Vanuatu) }\end{array}$ & $x$ & $x$ & & & $\begin{array}{l}\text { Bibliography } \\
\text { (EUR); Field } \\
\text { work (JDN) }\end{array}$ & EUR: CBNM, MO, P \\
\hline Ricinus communis L. & Euphorbiaceae & P caesp & $\begin{array}{l}\text { Supposed native from tropical } \\
\text { North East Africa; cultivated in } \\
\text { tropical regions }\end{array}$ & & $x$ & $x$ & & $\begin{array}{l}\text { Bibliography } \\
\text { (GLO); Field } \\
\text { work (JDN) }\end{array}$ & GLO: CBNM, MO, P \\
\hline $\begin{array}{l}\text { Salicornia } \\
\text { pachystachya Bunge } \\
\text { ex Ung.-Sternb. }\end{array}$ & Amaranthaceae & T succ & $\begin{array}{l}\text { Eastern Africa coastlines } \\
\text { (South Kenya to Natal), } \\
\text { Madagascar }\end{array}$ & $x$ & & & & $\begin{array}{l}\text { Field work } \\
\text { (EUR) }\end{array}$ & EUR: CBNM, MO \\
\hline $\begin{array}{l}\text { Salsola littoralis } \\
\text { Moq. }\end{array}$ & Amaranthaceae & $\begin{array}{l}\text { Ch I } \\
\text { succ }\end{array}$ & Madagascar & $x$ & & & & $\begin{array}{l}\text { Bibliography } \\
\text { (EUR) }\end{array}$ & EUR: CBNM, MO, P \\
\hline $\begin{array}{l}\text { Salvadora } \\
\text { angustifolia Turrill }\end{array}$ & Salvadoraceae & $P$ caesp & Madagascar & & $x$ & & & $\begin{array}{l}\text { Field work } \\
\text { (JDN) }\end{array}$ & JDN: CBNM, MO, P \\
\hline
\end{tabular}




\begin{tabular}{|c|c|c|c|c|c|c|c|c|c|}
\hline \multirow[t]{2}{*}{ Scientific name } & \multirow[t]{2}{*}{ Family } & \multirow{2}{*}{$\begin{array}{l}\text { Life } \\
\text { forms }\end{array}$} & \multirow{2}{*}{$\begin{array}{l}\text { General status and } \\
\text { distribution range }\end{array}$} & \multicolumn{4}{|c|}{ Presence } & \multirow{2}{*}{$\begin{array}{l}\text { First record } \\
\text { source from } \\
\text { (name of the } \\
\text { territory) }\end{array}$} & \multirow{2}{*}{$\begin{array}{l}\text { Voucher herbarium } \\
\text { specimens (name of the } \\
\text { territory and acronym of } \\
\text { the herbarium) }\end{array}$} \\
\hline & & & & $\begin{array}{c}\text { Europ } \\
\text { a } \\
\text { (EUR) }\end{array}$ & $\begin{array}{l}\text { Juan } \\
\text { de } \\
\text { Nova } \\
\text { (JDN) }\end{array}$ & $\begin{array}{l}\text { Iles } \\
\text { Glorieuses } \\
\text { (GLO) }\end{array}$ & $\begin{array}{l}\text { Tromelin } \\
\text { (TRO) }\end{array}$ & & \\
\hline $\begin{array}{l}\text { Scaevola taccada } \\
\text { (Gaertn.) Roxb. }\end{array}$ & Goodeniaceae & $\mathrm{P}$ caesp & $\begin{array}{l}\text { Indo-Pacific (Hawai to Africa } \\
\text { coastlines); introduced and } \\
\text { cultivated elsewhere }\end{array}$ & & $x$ & $x$ & & $\begin{array}{l}\text { Bibliography } \\
\text { (JDN, GLO) }\end{array}$ & $\begin{array}{l}\text { JDN: CBNM; GLO: CBNM, } \\
\text { MO, P }\end{array}$ \\
\hline $\begin{array}{l}\text { Sclerodactylon } \\
\text { macrostachyum } \\
\text { (Benth.) A. Camus }\end{array}$ & Poaceae & $\begin{array}{l}\text { Ch herb } \\
\text { caesp }\end{array}$ & $\begin{array}{l}\text { South Tanzania, Madagascar, } \\
\text { coral Seychelles }\end{array}$ & $x$ & $x$ & $x$ & & $\begin{array}{l}\text { Bibliography } \\
\text { (EUR, JDN, } \\
\text { GLO) }\end{array}$ & $\begin{array}{l}\text { EUR: CBNM, MO, P; JDN: } \\
\text { CBNM, MO, P; GLO: } \\
\text { CBNM, MO, P }\end{array}$ \\
\hline $\begin{array}{l}\text { Secamone } \\
\text { pachystigma Jum. \& } \\
\text { H. Perrier }\end{array}$ & Apocynaceae & st PL & $\begin{array}{l}\text { Madagascar, Comoros, coral } \\
\text { Seychelles }\end{array}$ & & & $x$ & & $\begin{array}{l}\text { Field work } \\
\text { (GLO) }\end{array}$ & GLO: CBNM, MO, P \\
\hline $\begin{array}{l}\text { Senna occidentalis } \\
\text { (L.) Link }\end{array}$ & Fabaceae & T scap & $\begin{array}{l}\text { Supposed native from South } \\
\text { America; became pantropical }\end{array}$ & & $x$ & $x$ & & $\begin{array}{l}\text { Field work } \\
\text { (JDN, GLO) }\end{array}$ & $\begin{array}{l}\text { JDN: CBNM, MO; GLO: } \\
\text { CBNM, MO, P }\end{array}$ \\
\hline Senna sp. & Fabaceae & $\mathrm{P}$ caesp & $?$ & & $x$ & & & $\begin{array}{l}\text { Field work } \\
\text { (JDN) }\end{array}$ & JDN: CBNM \\
\hline $\begin{array}{l}\text { Sesuvium } \\
\text { portulacastrum (L.) L. }\end{array}$ & Aizoaceae & $\begin{array}{l}\text { Ch I } \\
\text { succ }\end{array}$ & Pantropical coastlines & $x$ & & $\mathrm{x}$ & & $\begin{array}{l}\text { Bibliography } \\
\text { (EUR, GLO) }\end{array}$ & EUR: CBNM \\
\hline Sida acuta Burm. f. & Malvaceae & $\begin{array}{l}\text { Ch suff } \\
\text { caesp / } \\
\text { T caesp }\end{array}$ & $\begin{array}{l}\text { Pantropical, extended to } \\
\text { South West and South East } \\
\text { Africa }\end{array}$ & $x$ & & $x$ & & $\begin{array}{l}\text { Field work } \\
\text { (EUR, GLO) }\end{array}$ & $\begin{array}{l}\text { EUR: CBNM, MO, P; GLO: } \\
\text { CBNM, MO, P }\end{array}$ \\
\hline $\begin{array}{l}\text { Sida cordifolia L. } \\
\text { subsp. cordifolia }\end{array}$ & Malvaceae & $\begin{array}{l}\text { Ch suff } \\
\text { caesp / } \\
\text { T caesp }\end{array}$ & $\begin{array}{l}\text { Asia tropical; considered } \\
\text { introduced elsewhere, but } \\
\text { probably native to Seychelles }\end{array}$ & $x$ & & & & $\begin{array}{l}\text { Field work } \\
\text { (EUR) }\end{array}$ & EUR: CBNM, MO, P \\
\hline Sida pusilla Cav. s. I. & Malvaceae & $\begin{array}{l}\text { Ch frut } \\
\text { rept }\end{array}$ & Indo-Pacific & $x$ & $x$ & $x$ & $x$ & $\begin{array}{l}\text { Bibliography } \\
\text { (EUR, GLO, } \\
\text { TRO); Field } \\
\text { work (JDN) }\end{array}$ & $\begin{array}{l}\text { EUR: CBNM, MO, P; JDN: } \\
\text { CBNM, MO; GLO: } \\
\text { CBNM, MO, P; TRO: } \\
\text { CBNM, MO, P }\end{array}$ \\
\hline Sida rhombifolia L. & Malvaceae & $\begin{array}{l}\text { Ch suff } \\
\text { caesp / } \\
\text { P caesp }\end{array}$ & Pantropical & & & $x$ & & $\begin{array}{l}\text { Bibliography } \\
\text { (GLO) }\end{array}$ & \\
\hline
\end{tabular}




\begin{tabular}{|c|c|c|c|c|c|c|c|c|c|}
\hline \multirow[t]{2}{*}{ Scientific name } & \multirow[t]{2}{*}{ Family } & \multirow{2}{*}{$\begin{array}{l}\text { Life } \\
\text { forms }\end{array}$} & \multirow{2}{*}{$\begin{array}{l}\text { General status and } \\
\text { distribution range }\end{array}$} & \multicolumn{4}{|c|}{ Presence } & \multirow{2}{*}{$\begin{array}{l}\text { First record } \\
\text { source from } \\
\text { (name of the } \\
\text { territory) }\end{array}$} & \multirow{2}{*}{$\begin{array}{l}\text { Voucher herbarium } \\
\text { specimens (name of the } \\
\text { territory and acronym of } \\
\text { the herbarium) }\end{array}$} \\
\hline & & & & $\begin{array}{l}\text { Europ } \\
\text { a } \\
\text { (EUR) }\end{array}$ & $\begin{array}{l}\text { Juan } \\
\text { de } \\
\text { Nova } \\
\text { (JDN) }\end{array}$ & $\begin{array}{l}\text { Iles } \\
\text { Glorieuses } \\
\text { (GLO) }\end{array}$ & $\begin{array}{l}\text { Tromelin } \\
\text { (TRO) }\end{array}$ & & \\
\hline Sideroxylon inerme L. & Sapotaceae & P scap & $\begin{array}{l}\text { Eastern and Southern Africa, } \\
\text { Comoros, Seychelles }\end{array}$ & & $x$ & & & $\begin{array}{l}\text { Bibliography } \\
\text { (JDN) }\end{array}$ & JDN: CBNM, MO, P \\
\hline $\begin{array}{l}\text { Solanum } \\
\text { americanum Mill. }\end{array}$ & Solanaceae & T caesp & $\begin{array}{l}\text { Cosmopolitan tropical and } \\
\text { subtropical }\end{array}$ & $x$ & $x$ & $x$ & & $\begin{array}{l}\text { Field work } \\
\text { (EUR, JDN, } \\
\text { GLO) }\end{array}$ & $\begin{array}{l}\text { EUR: CBNM, MO, P; GLO: } \\
\text { CBNM, MO, P }\end{array}$ \\
\hline $\begin{array}{l}\text { Solanum } \\
\text { lycopersicum L. }\end{array}$ & Solanaceae & T caesp & $\begin{array}{l}\text { Central and South America; } \\
\text { widely cultivated in } \\
\text { temperates and warm } \\
\text { regions, often escaped and } \\
\text { naturalized }\end{array}$ & $x$ & & $x$ & & $\begin{array}{l}\text { Field work } \\
\text { (EUR, GLO) }\end{array}$ & EUR: CBNM \\
\hline Solanum sp. & Solanaceae & T caesp & $?$ & & & $x$ & & $\begin{array}{l}\text { Field work } \\
\text { (GLO) }\end{array}$ & GLO: CBNM, MO, P \\
\hline Sonchus oleraceus L. & Asteraceae & T sem & $\begin{array}{l}\text { Eurasia, North Africa, North } \\
\text { and West Asia }\end{array}$ & $x$ & & & & $\begin{array}{l}\text { Field work } \\
\text { (EUR) }\end{array}$ & \\
\hline $\begin{array}{l}\text { Sophora tomentosa } \\
\text { L. subsp. tomentosa }\end{array}$ & Fabaceae & $P$ caesp & Indo-Pacific & & & $x$ & & $\begin{array}{l}\text { Bibliography } \\
\text { (GLO) }\end{array}$ & GLO: CBNM, MO, P \\
\hline $\begin{array}{l}\text { Sporobolus virginicus } \\
\text { (L.) Kunth }\end{array}$ & Poaceae & $\begin{array}{l}\text { Ch herb } \\
\text { caesp / } \\
\text { c G rhiz }\end{array}$ & Pantropical to pansubtropical & & $x$ & $x$ & & $\begin{array}{l}\text { Bibliography } \\
\text { (GLO); Field } \\
\text { work (JDN) }\end{array}$ & $\begin{array}{l}\text { JDN: CBNM, MO, P; GLO: } \\
\text { CBNM, MO, P }\end{array}$ \\
\hline $\begin{array}{l}\text { Stachytarpheta } \\
\text { jamaicensis (L.) Vahl }\end{array}$ & Verbenaceae & $\begin{array}{l}\text { Ch suff } \\
\text { caesp }\end{array}$ & $\begin{array}{l}\text { America (West Indies and } \\
\text { South of the United-States of } \\
\text { America to Mexico, Ecuador } \\
\text { and Brazil); became } \\
\text { pantropical }\end{array}$ & & & $x$ & & $\begin{array}{l}\text { Bibliography } \\
\text { (GLO) }\end{array}$ & GLO: CBNM, MO, P \\
\hline $\begin{array}{l}\text { Stachytarpheta } \\
\text { urticifolia Sims }\end{array}$ & Verbenaceae & $\begin{array}{l}\text { Ch suff } \\
\text { caesp }\end{array}$ & $\begin{array}{l}\text { Supposed native from South- } \\
\text { East Asia ; introduced and } \\
\text { naturalized elsewhere }\end{array}$ & & & $x$ & & $\begin{array}{l}\text { Field work } \\
\text { (GLO) }\end{array}$ & GLO: CBNM, MO, P \\
\hline
\end{tabular}




\begin{tabular}{|c|c|c|c|c|c|c|c|c|c|}
\hline \multirow[t]{2}{*}{ Scientific name } & \multirow[t]{2}{*}{ Family } & \multirow{2}{*}{$\begin{array}{l}\text { Life } \\
\text { forms }\end{array}$} & \multirow{2}{*}{$\begin{array}{l}\text { General status and } \\
\text { distribution range }\end{array}$} & \multicolumn{4}{|c|}{ Presence } & \multirow{2}{*}{$\begin{array}{l}\text { First record } \\
\text { source from } \\
\text { (name of the } \\
\text { territory) }\end{array}$} & \multirow{2}{*}{$\begin{array}{l}\text { Voucher herbarium } \\
\text { specimens (name of the } \\
\text { territory and acronym of } \\
\text { the herbarium) }\end{array}$} \\
\hline & & & & $\begin{array}{l}\text { Europ } \\
\quad \text { a } \\
\text { (EUR) }\end{array}$ & $\begin{array}{l}\text { Juan } \\
\text { de } \\
\text { Nova } \\
\text { (JDN) }\end{array}$ & $\begin{array}{l}\text { Iles } \\
\text { Glorieuses } \\
\text { (GLO) }\end{array}$ & $\begin{array}{l}\text { Tromelin } \\
\text { (TRO) }\end{array}$ & & \\
\hline $\begin{array}{l}\text { Stenotaphrum } \\
\text { micranthum (Desv.) } \\
\text { C.E. Hubb., }\end{array}$ & Poaceae & H rept & $\begin{array}{l}\text { Indo-Pacific coastlines, } \\
\text { Seychelles, Mascarene islands }\end{array}$ & & & $\mathrm{x}$ & & $\begin{array}{l}\text { Field work } \\
\text { (GLO) }\end{array}$ & GLO: CBNM, MO, P \\
\hline $\begin{array}{l}\text { Striga asiatica (L.) } \\
\text { Kuntze }\end{array}$ & Orobanchaceae & T scap & $\begin{array}{l}\text { Warm regions of the Old } \\
\text { World and United-States of } \\
\text { America }\end{array}$ & $x$ & & $x$ & & $\begin{array}{l}\text { Bibliography } \\
\text { (EUR); Field } \\
\text { work (GLO) }\end{array}$ & $\begin{array}{l}\text { EUR: CBNM, MO, P; GLO: } \\
\text { CBNM, MO, P }\end{array}$ \\
\hline $\begin{array}{l}\text { Suaeda monoica } \\
\text { Forssk. ex J.F. Gmel. }\end{array}$ & Amaranthaceae & $\begin{array}{l}\text { Ch I } \\
\text { succ }\end{array}$ & $\begin{array}{l}\text { Eastern Africa to Egypt, Saudi } \\
\text { Arabia, Sri Lanka, India } \\
\text { (Madras), Madagascar, Israel, } \\
\text { Syria }\end{array}$ & $x$ & & & & $\begin{array}{l}\text { Field work } \\
\text { (EUR) }\end{array}$ & \\
\hline Suriana maritima L. & Surianaceae & P caesp & $\begin{array}{l}\text { Pantropical discontinuous } \\
\text { distribution }\end{array}$ & $x$ & $x$ & $X$ & & $\begin{array}{l}\text { Bibliography } \\
\text { (EUR, GLO); } \\
\text { Field work } \\
\text { (JDN) }\end{array}$ & $\begin{array}{l}\text { EUR: CBNM, MO, P; JDN: } \\
\text { CBNM, MO; GLO: CBNM, } \\
\text { MO, P }\end{array}$ \\
\hline $\begin{array}{l}\text { Tabebuia pallida } \\
\text { (Lindl.) Miers }\end{array}$ & Bignoniaceae & P scap & West Indies & & $x$ & & & $\begin{array}{l}\text { Field work } \\
\text { (JDN) }\end{array}$ & \\
\hline Tamarindus indica L. & Fabaceae & P scap & $\begin{array}{l}\text { Indigeneous incertain area } \\
\text { (tropical Africa, Madagascar, } \\
\text { India); became paleotropical; } \\
\text { widely culivated in tropical } \\
\text { regions }\end{array}$ & $x$ & $x$ & & $x$ & $\begin{array}{l}\text { Bibliography } \\
\text { (EUR); Field } \\
\text { work (JDN, } \\
\text { TRO) }\end{array}$ & \\
\hline $\begin{array}{l}\text { Tecticornia indica } \\
\text { (Willd.) K.A. Sheph. \& } \\
\text { Paul G. Wilson }\end{array}$ & Amaranthaceae & $\begin{array}{l}\text { Ch I } \\
\text { succ }\end{array}$ & $\begin{array}{l}\text { Tropical coastlines of the } \\
\text { Indian Ocean }\end{array}$ & $x$ & & & & $\begin{array}{l}\text { Bibliography } \\
\text { (EUR) }\end{array}$ & EUR: CBNM, MO, P \\
\hline $\begin{array}{l}\text { Tephrosia purpurea } \\
\text { (L.) Pers. subsp. } \\
\text { dunensis Brummitt }\end{array}$ & Fabaceae & $\begin{array}{l}\text { Ch suff } \\
\text { caesp / } \\
\text { T caesp }\end{array}$ & $\begin{array}{l}\text { Eastern Africa, Madagascar, } \\
\text { Comoros }\end{array}$ & & $x$ & & & $\begin{array}{l}\text { Field work } \\
\text { (JDN) }\end{array}$ & JDN: CBNM, MO, P \\
\hline
\end{tabular}




\begin{tabular}{|c|c|c|c|c|c|c|c|c|c|}
\hline \multirow[t]{2}{*}{ Scientific name } & \multirow[t]{2}{*}{ Family } & \multirow{2}{*}{$\begin{array}{l}\text { Life } \\
\text { forms }\end{array}$} & \multirow{2}{*}{$\begin{array}{l}\text { General status and } \\
\text { distribution range }\end{array}$} & \multicolumn{4}{|c|}{ Presence } & \multirow{2}{*}{$\begin{array}{l}\text { First record } \\
\text { source from } \\
\text { (name of the } \\
\text { territory) }\end{array}$} & \multirow{2}{*}{$\begin{array}{l}\text { Voucher herbarium } \\
\text { specimens (name of the } \\
\text { territory and acronym of } \\
\text { the herbarium) }\end{array}$} \\
\hline & & & & $\begin{array}{l}\text { Europ } \\
\text { a } \\
\text { (EUR) }\end{array}$ & $\begin{array}{l}\text { Juan } \\
\text { de } \\
\text { Nova } \\
\text { (JDN) }\end{array}$ & $\begin{array}{l}\text { lles } \\
\text { Glorieuses } \\
\text { (GLO) }\end{array}$ & $\begin{array}{l}\text { Tromelin } \\
\text { (TRO) }\end{array}$ & & \\
\hline Terminalia catappa L. & Combretaceae & P scap & $\begin{array}{l}\text { Indo-Pacific coastlines } \\
\text { (indigeneous area discussed } \\
\text { for the western part of the } \\
\text { Indian Ocean); widely } \\
\text { cultivated in tropical regions }\end{array}$ & & $x$ & $x$ & $x$ & $\begin{array}{l}\text { Bibliography } \\
\text { (GLO); Field } \\
\text { work (JDN, } \\
\text { TRO) }\end{array}$ & $\begin{array}{l}\text { GLO: CBNM, MO, P; TRO: } \\
\text { CBNM,MO, P }\end{array}$ \\
\hline $\begin{array}{l}\text { Thespesia } \\
\text { populneoides (Roxb.) } \\
\text { Kostel. }\end{array}$ & Malvaceae & P scap & $\begin{array}{l}\text { Indian Ocean coastlines } \\
\text { (Mozambique, Zanzibar to } \\
\text { Malaysia and Australia) }\end{array}$ & $x$ & $x$ & $x$ & & $\begin{array}{l}\text { Bibliography } \\
\text { (EUR, GLO); } \\
\text { Field work } \\
\text { (JDN) }\end{array}$ & $\begin{array}{l}\text { EUR: CBNM, MO, P; JDN: } \\
\text { CBNM, MO, P; GLO: } \\
\text { CBNM, MO, P }\end{array}$ \\
\hline $\begin{array}{l}\text { Tradescantia } \\
\text { spathacea Sw. }\end{array}$ & Commelinaceae & $\begin{array}{l}\text { Ch herb } \\
\text { caesp }\end{array}$ & $\begin{array}{l}\text { Central America; cultivated } \\
\text { and naturalized in tropical } \\
\text { regions }\end{array}$ & $x$ & $x$ & & & $\begin{array}{l}\text { Field work } \\
\text { (EUR, JDN) }\end{array}$ & \\
\hline $\begin{array}{l}\text { Trianthema } \\
\text { portulacastrum L. }\end{array}$ & Aizoaceae & T rept & America; became pantropical & $x$ & $x$ & & & $\begin{array}{l}\text { Field work } \\
\text { (EUR, JDN) }\end{array}$ & EUR: CBNM, MO, P \\
\hline Tribulus cistoides L. & Zygophyllaceae & Trept & $\begin{array}{l}\text { East and North East of Africa, } \\
\text { Madagascar, Comoros, } \\
\text { Mascarene islands (Reunion } \\
\text { and Mauritius), Cape Verde, } \\
\text { warm regions of America }\end{array}$ & & $x$ & $x$ & & $\begin{array}{l}\text { Bibliography } \\
\text { (JDN, GLO) }\end{array}$ & $\begin{array}{l}\text { JDN: CBNM, MO; GLO: } \\
\text { CBNM, MO, P }\end{array}$ \\
\hline $\begin{array}{l}\text { Tribulus parvispinus } \\
\text { C. Presl }\end{array}$ & Zygophyllaceae & T rept & $\begin{array}{l}\text { Coastlines regions from South } \\
\text { Africa to Saudi Arabia and } \\
\text { Pakistan, Madagascar }\end{array}$ & $x$ & & & & $\begin{array}{l}\text { Bibliography } \\
\text { (EUR) }\end{array}$ & EUR: CBNM, MO, P \\
\hline $\begin{array}{l}\text { Tricholaena } \\
\text { monachne (Trin.) } \\
\text { Stapf \& C.E. Hubb. }\end{array}$ & Poaceae & H caesp & $\begin{array}{l}\text { Southern and tropical Africa, } \\
\text { Madagascar, Mascarene } \\
\text { islands (Reunion and } \\
\text { Mauritius) }\end{array}$ & $\mathrm{x}$ & $x$ & & & $\begin{array}{l}\text { Bibliography } \\
\text { (JDN); Field } \\
\text { work (EUR) }\end{array}$ & $\begin{array}{l}\text { EUR: CBNM, MO, P; JDN: } \\
\text { CBNM, MO, P }\end{array}$ \\
\hline
\end{tabular}




\begin{tabular}{|c|c|c|c|c|c|c|c|c|c|}
\hline \multirow[t]{2}{*}{ Scientific name } & \multirow[t]{2}{*}{ Family } & \multirow{2}{*}{$\begin{array}{l}\text { Life } \\
\text { forms }\end{array}$} & \multirow{2}{*}{$\begin{array}{l}\text { General status and } \\
\text { distribution range }\end{array}$} & \multicolumn{4}{|c|}{ Presence } & \multirow{2}{*}{$\begin{array}{l}\text { First record } \\
\text { source from } \\
\text { (name of the } \\
\text { territory) }\end{array}$} & \multirow{2}{*}{$\begin{array}{l}\text { Voucher herbarium } \\
\text { specimens (name of the } \\
\text { territory and acronym o } \\
\text { the herbarium) }\end{array}$} \\
\hline & & & & $\begin{array}{l}\text { Europ } \\
\text { a } \\
\text { (EUR) }\end{array}$ & $\begin{array}{l}\text { Juan } \\
\text { de } \\
\text { Nova } \\
\text { (JDN) }\end{array}$ & $\begin{array}{l}\text { Iles } \\
\text { Glorieuses } \\
\text { (GLO) }\end{array}$ & $\begin{array}{l}\text { Tromelin } \\
\text { (TRO) }\end{array}$ & & \\
\hline Tridax procumbens L. & Asteraceae & $\begin{array}{l}\text { Ch herb } \\
\text { rept }\end{array}$ & $\begin{array}{l}\text { Central America; became } \\
\text { pantropical }\end{array}$ & & $x$ & $x$ & & $\begin{array}{l}\text { Bibliography } \\
\text { (GLO); Field } \\
\text { work (JDN) }\end{array}$ & $\begin{array}{l}\text { JDN: CBNM, MO, P; GLO: } \\
\text { CBNM, MO, P }\end{array}$ \\
\hline $\begin{array}{l}\text { Triumfetta } \\
\text { procumbens G. Forst. }\end{array}$ & Malvaceae & $\begin{array}{l}\text { Ch suff } \\
\text { rept }\end{array}$ & $\begin{array}{l}\text { Seychelles, Tropical Asia, } \\
\text { Pacific }\end{array}$ & & & & $x$ & $\begin{array}{l}\text { Field work } \\
\text { (TRO) }\end{array}$ & TRO: CBNM, MO, P \\
\hline $\begin{array}{l}\text { Urochloa deflexa } \\
\text { (Schumach.) H. } \\
\text { Scholz }\end{array}$ & Poaceae & T caesp & $\begin{array}{l}\text { Tropical and southern Africa, } \\
\text { tropical Arabia, India }\end{array}$ & & $x$ & & & $\begin{array}{l}\text { Field work } \\
\text { (JDN) }\end{array}$ & JDN: CBNM, MO, P \\
\hline $\begin{array}{l}\text { Urochloa maxima } \\
\text { (Jacq.) R.D. Webster }\end{array}$ & Poaceae & $\begin{array}{l}\text { H caesp } \\
\text { / Ch } \\
\text { herb } \\
\text { caesp }\end{array}$ & $\begin{array}{l}\text { Tropical Africa; widely } \\
\text { introduced and naturalized in } \\
\text { warm regions }\end{array}$ & $x$ & $x$ & $x$ & & $\begin{array}{l}\text { Bibliography } \\
\text { (EUR); Field } \\
\text { work (JDN, } \\
\text { GLO) }\end{array}$ & $\begin{array}{l}\text { EUR: CBNM, MO, P; GLO: } \\
\text { CBNM, MO, P }\end{array}$ \\
\hline $\begin{array}{l}\text { Vachellia farnesiana } \\
\text { (L.) Wight \& Arn. }\end{array}$ & Fabaceae & P caesp & $\begin{array}{l}\text { Native from tropical America; } \\
\text { introduced and naturalized in } \\
\text { all the tropics, now } \\
\text { pantropical }\end{array}$ & & $x$ & & & $\begin{array}{l}\text { Field work } \\
\text { (JDN) }\end{array}$ & \\
\hline $\begin{array}{l}\text { Vigna marina (Burm.) } \\
\text { Merr. }\end{array}$ & Fabaceae & $\begin{array}{l}\text { Ch herb } \\
\text { rept / st } \\
\text { PL }\end{array}$ & Pantropical (coastlines) & & $x$ & $x$ & & $\begin{array}{l}\text { Field work } \\
\text { (JDN, GLO) }\end{array}$ & $\begin{array}{l}\text { JDN: CBNM; GLO: CBNM, } \\
\text { MO, P }\end{array}$ \\
\hline $\begin{array}{l}\text { Wollastonia biflora } \\
\text { (L.) DC. }\end{array}$ & Asteraceae & $\begin{array}{l}\text { Ch herb } \\
\text { rept / } \\
\text { Ch herb } \\
\text { caesp }\end{array}$ & $\begin{array}{l}\text { Indian Ocean coastlines } \\
\text { (Eastern Africa to India and } \\
\text { Australia) }\end{array}$ & & $x$ & $x$ & & $\begin{array}{l}\text { Bibliography } \\
\text { (JDN, GLO) }\end{array}$ & $\begin{array}{l}\text { JDN: CBNM, MO, P; GLO: } \\
\text { CBNM, MO, P }\end{array}$ \\
\hline $\begin{array}{l}\text { Zaleya cf. camillei } \\
\text { (Cordem.) H.E.K. } \\
\text { Hartmann }\end{array}$ & Aizoaceae & Trept & $\begin{array}{l}\text { Madagascar, Mascarene } \\
\text { islands (Reunion) }\end{array}$ & $x$ & & & & $\begin{array}{l}\text { Bibliography } \\
\text { (EUR) }\end{array}$ & EUR: CBNM, MO, P \\
\hline Zea mays L. & Poaceae & T caesp & $\begin{array}{l}\text { Central America (artificial } \\
\text { origin); cosmopolitan culture }\end{array}$ & & $x$ & $x$ & & $\begin{array}{l}\text { Bibliography } \\
\text { (JDN, GLO) }\end{array}$ & \\
\hline
\end{tabular}




\section{APPENDIX B}

Table 3. List of taxa found on Europa. In the column 'General status on Europa' differents codes were used : A = alien ; N = native ; K = cryptogenic.

\begin{tabular}{|c|c|c|c|c|}
\hline Scientific name & Family & $\begin{array}{c}\text { General status on } \\
\text { Europa }\end{array}$ & $\begin{array}{c}\text { Population and cultural status on } \\
\text { Europa }\end{array}$ & $\begin{array}{c}\text { Distribution range } \\
\text { on Europa }\end{array}$ \\
\hline Abutilon pseudocleistogamum Hochr. & Malvaceae & A & naturalized & local \\
\hline Achyranthes aspera L. var. aspera & Amaranthaceae & $\mathrm{K}$ & native or naturalized & local \\
\hline Achyranthes sp.nov.? & Amaranthaceae & $\mathrm{N}$ & native & local \\
\hline Achyranthes cf. talbotii Hutch. \& Dalziel & Amaranthaceae & $\mathrm{N}$ & native & local \\
\hline Agave sisalana Perrine & Asparagaceae & $A$ & invasive, cultivated & local \\
\hline Alternanthera pungens Kunth & Amaranthaceae & A & naturalized & local \\
\hline Amaranthus graecizans L. subsp. silvestris (Vill.) Brenan & Amaranthaceae & A & naturalized & local \\
\hline Amaranthus viridis $\mathrm{L}$. & Amaranthaceae & $A$ & naturalized & local \\
\hline Avicennia marina (Forssk.) Vierh. & Avicenniaceae & $\mathrm{N}$ & native & local \\
\hline Boerhavia coccinea Mill. & Nyctaginaceae & $\mathrm{K}$ & native or naturalized & wide \\
\hline Boerhavia erecta $\mathrm{L}$. & Nyctaginaceae & $A$ & naturalized & supposed extinct \\
\hline Boerhavia repens $\mathrm{L}$. & Nyctaginaceae & $\mathrm{N}$ & native & local \\
\hline Bothriochloa pertusa (L.) A. Camus & Poaceae & $A$ & naturalized & local \\
\hline Bougainvillea $x$ buttiana Holttum \& Standl. & Nyctaginaceae & $A$ & not naturalized, cultivated & supposed extinct \\
\hline Bruguiera gymnorhiza (L.) Savigny & Rhizophoraceae & $\mathrm{N}$ & native & local \\
\hline Capparis cartilaginea Decne. & Capparaceae & $\mathrm{N}$ & native & wide \\
\hline Capsicum frutescens $\mathrm{L}$. & Solanaceae & $A$ & naturalized, cultivated & supposed extinct \\
\hline Casuarina equisetifolia $\mathrm{L}$. & Casuarinaceae & $A$ & invasive, cultivated & wide \\
\hline Ceriops tagal (Perr.) C.B. Rob. & Rhizophoraceae & $\mathrm{N}$ & native & local \\
\hline Chloris barbata Sw. & Poaceae & $A$ & naturalized & local \\
\hline Cocos nucifera $\mathrm{L}$. & Arecaceae & $A$ & not naturalized, cultivated & local \\
\hline Corchorus trilocularis L. & Malvaceae & $A$ & naturalized & local \\
\hline Cordia subcordata Lam. & Boraginaceae & $\mathrm{N}$ & native & local \\
\hline Cucumis melo L. subsp. agrestis (Naudin) Pangalo var. agrestis & Cucurbitaceae & $\mathrm{K}$ & native or naturalized & local \\
\hline Cynanchum luteifluens (Jum. \& H. Perrier) Desc. & Apocynaceae & $\mathrm{N}$ & native & wide \\
\hline Cynodon dactylon (L.) Pers. & Poaceae & $A$ & naturalized & local \\
\hline Cyperus rotundus L. & Cyperaceae & A & naturalized & local \\
\hline Dactyloctenium aegyptium (L.) Willd. & Poaceae & A & naturalized & local \\
\hline Dactyloctenium capitatum A. Camus & Poaceae & $\mathrm{N}$ & native & wide \\
\hline
\end{tabular}




\begin{tabular}{|c|c|c|c|c|}
\hline Scientific name & Family & $\begin{array}{l}\text { General status on } \\
\text { Europa }\end{array}$ & $\begin{array}{c}\text { Population and cultural status on } \\
\text { Europa }\end{array}$ & $\begin{array}{l}\text { Distribution range } \\
\text { on Europa }\end{array}$ \\
\hline Dactyloctenium ctenioides (Steud.) Lorch ex Bosser & Poaceae & $\mathrm{K}$ & native or naturalized & local \\
\hline Daknopholis boivinii (A. Camus) Clayton & Poaceae & $\mathrm{N}$ & native & wide \\
\hline Delonix regia (Bojer) Raf. & Fabaceae & A & naturalized, cultivated & local \\
\hline Digitaria ciliaris (Retz.) Koeler var. chrysoblephara (Fig. \& De Not.) & Poaceae & A & naturalized & local \\
\hline Eragrostis capuronii A. Camus & Poaceae & $\mathrm{N}$ & native & wide \\
\hline Eragrostis ciliaris (L.) R. Br. & Poaceae & $\mathrm{N}$ & native & wide \\
\hline Euphorbia hirta L. & Euphorbiaceae & $A$ & naturalized & local \\
\hline Euphorbia prostrata Aiton & Euphorbiaceae & $A$ & naturalized & wide \\
\hline Euphorbia sp. nov.? & Euphorbiaceae & $\mathrm{N}$ & native & wide \\
\hline Euphorbia stenoclada Baill. & Euphorbiaceae & $\mathrm{N}$ & native & wide \\
\hline Ficus marmorata Bojer ex Baker & Moraceae & $\mathrm{N}$ & native & wide \\
\hline Fimbristylis cymosa R. Br. s. I. & Cyperaceae & $\mathrm{N}$ & native & local \\
\hline Furcraea foetida (L.) Haw. & Asparagaceae & A & invasive, cultivated & local \\
\hline Guettarda speciosa L. & Rubiaceae & $\mathrm{N}$ & native & local \\
\hline Guilandina bonduc L. & Fabaceae & $\mathrm{N}$ & native & local \\
\hline Hibiscus tiliaceus L. & Malvaceae & $\mathrm{N}$ & native & local \\
\hline Ipomoea coptica (L.) Roth & Convolvulaceae & $A$ & naturalized & local \\
\hline Ipomoea pes-caprae (L.) R. Br. subsp. brasiliensis (L.) Ooststr. & Convolvulaceae & $\mathrm{N}$ & native & local \\
\hline Ipomoea violacea $\mathrm{L}$. & Convolvulaceae & $\mathrm{N}$ & native & local \\
\hline Lepidium englerianum (Muschl.) Al-Shehbaz & Brassicaceae & $\mathrm{N}$ & native & wide \\
\hline Lycium elliotii Dammer s. I. & Solanaceae & $\mathrm{N}$ & native & local \\
\hline Malvastrum coromandelianum (L.) Garcke & Malvaceae & A & naturalized & local \\
\hline Mimusops caffra E. Mey. ex A. DC. & Sapotaceae & A & not naturalized, cultivated & supposed extinct \\
\hline Mollugo nudicaulis Lam. & Molluginaceae & $\mathrm{N}$ & native & local \\
\hline Moringa oleifera Lam. & Moringaceae & $A$ & naturalized, cultivated & local \\
\hline Oldenlandia corymbosa L. var. caespitosa (Benth.) Verdc. & Rubiaceae & A & naturalized & local \\
\hline Oldenlandia corymbosa L. var. corymbosa & Rubiaceae & A & naturalized & local \\
\hline Ophioglossum lancifolium C. Presl & Ophioglossaceae & $\mathrm{N}$ & native & local \\
\hline Ophioglossum polyphyllum A. Braun & Ophioglossaceae & $\mathrm{N}$ & native & local \\
\hline Panicum pseudowoeltzkowii A. Camus & Poaceae & $\mathrm{K}$ & native or naturalized & local \\
\hline Panicum voeltzkowii Mez s. I. & Poaceae & $\mathrm{N}$ & native & wide \\
\hline Pemphis acidula J.R. Forst. \& G. Forst. & Lythraceae & $\mathrm{N}$ & native & wide \\
\hline
\end{tabular}




\begin{tabular}{|c|c|c|c|c|}
\hline Scientific name & Family & $\begin{array}{l}\text { General status on } \\
\text { Europa }\end{array}$ & $\begin{array}{c}\text { Population and cultural status on } \\
\text { Europa }\end{array}$ & $\begin{array}{l}\text { Distribution range } \\
\text { on Europa }\end{array}$ \\
\hline Phoenix dactylifera $\mathrm{L}$. & Arecaceae & A & not naturalized, cultivated & local \\
\hline Phyllanthus maderaspatensis L. s. I. & Phyllanthaceae & $\mathrm{N}$ & native & wide \\
\hline Phyllanthus sp.1 & Phyllanthaceae & $\mathrm{N}$ & native & local \\
\hline Phyllanthus sp.2 & Phyllanthaceae & $\mathrm{N}$ & native & local \\
\hline Pisonia grandis $\mathrm{R}$. Br. & Nyctaginaceae & $\mathrm{N}$ & native & local \\
\hline Plumbago aphylla Boj. ex Boiss. & Plumbaginaceae & $\mathrm{N}$ & native & wide \\
\hline Portulaca granulatostellulata (Poelln.) Ricceri \& Arrigoni & Portulacaceae & $\mathrm{K}$ & native or naturalized & local \\
\hline Portulaca nitida (Danin et H.G. Baker) Ricceri et Arrigoni & Portulacaceae & $\mathrm{N}$ & native & local \\
\hline Portulaca aff. tuberosa Roxb. & Portulacaceae & $\mathrm{N}$ & native & local \\
\hline Psiadia altissima (DC.) Drake & Asteraceae & $\mathrm{N}$ & native & wide \\
\hline Rhizophora mucronata Lam. & Rhizophoraceae & $\mathrm{N}$ & native & local \\
\hline Salicornia pachystachya Bunge ex Ung.-Sternb. & Amaranthaceae & $\mathrm{N}$ & native & local \\
\hline Salsola littoralis Moq. & Amaranthaceae & $\mathrm{N}$ & native & wide \\
\hline Sclerodactylon macrostachyum (Benth.) A. Camus & Poaceae & $\mathrm{N}$ & native & wide \\
\hline Sesuvium portulacastrum (L.) L. & Aizoaceae & $\mathrm{N}$ & native & local \\
\hline Sida acuta Burm. f. & Malvaceae & $A$ & naturalized & local \\
\hline Sida cordifolia L. subsp. cordifolia & Malvaceae & $A$ & naturalized & local \\
\hline Sida pusilla Cav. s. I. & Malvaceae & $A$ & naturalized & local \\
\hline Solanum americanum Mill. & Solanaceae & $A$ & naturalized & local \\
\hline Solanum lycopersicum L. & Solanaceae & $A$ & naturalized & local \\
\hline Sonchus oleraceus L. & Asteraceae & $A$ & naturalized & supposed extinct \\
\hline Striga asiatica (L.) Kuntze & Orobanchaceae & $A$ & naturalized & local \\
\hline Suaeda monoica Forssk. ex J.F. Gmel. & Amaranthaceae & $\mathrm{N}$ & native & local \\
\hline Suriana maritima L. & Surianaceae & $\mathrm{N}$ & native & wide \\
\hline Tamarindus indica $\mathrm{L}$. & Fabaceae & $A$ & not naturalized, cultivated & supposed extinct \\
\hline Tecticornia indica (Willd.) K.A. Sheph. \& Paul G. Wilson & Amaranthaceae & $\mathrm{N}$ & native & local \\
\hline Thespesia populneoides (Roxb.) Kostel. & Malvaceae & $\mathrm{N}$ & native & local \\
\hline Tradescantia spathacea Sw. & Commelinaceae & A & not naturalized, cultivated & supposed extinct \\
\hline Trianthema portulacastrum $\mathrm{L}$. & Aizoaceae & A & naturalized & local \\
\hline Tribulus parvispinus C. Presl & Zygophyllaceae & $\mathrm{K}$ & native or naturalized & local \\
\hline Tricholaena monachne (Trin.) Stapf \& C.E. Hubb. & Poaceae & $\mathrm{N}$ & native & local \\
\hline Urochloa maxima (Jacq.) R.D. Webster & Poaceae & $A$ & naturalized & local \\
\hline Zaleya cf. camillei (Cordem.) H.E.K. Hartmann & Aizoaceae & $\mathrm{K}$ & native or naturalized & local \\
\hline
\end{tabular}


Table 4. List of taxa found on Juan de Nova. In the column 'General status on Europa' differents codes were used : A = alien ; $\mathrm{N}=$ native ; $\mathrm{K}=$ cryptogenic.

\begin{tabular}{|c|c|c|c|c|}
\hline Scientific name & Family & $\begin{array}{c}\text { General status on } \\
\text { Juan de Nova }\end{array}$ & $\begin{array}{c}\text { Population and cultural status on } \\
\text { Juan de Nova }\end{array}$ & $\begin{array}{c}\text { Distribution range } \\
\text { on Juan de Nova }\end{array}$ \\
\hline$\overline{\text { Acalypha indica } \mathrm{L} \text {. }}$ & Euphorbiaceae & A & naturalized & local \\
\hline Acanthocereus tetragonus (L.) Hummelinck & Cactaceae & A & naturalized, cultivated & local \\
\hline Achyranthes aspera L. var. aspera & Amaranthaceae & $\mathrm{N}$ & native & local \\
\hline Achyranthes aspera L. var. fruticosa (Lam.) Boerl. & Amaranthaceae & $\mathrm{N}$ & native & local \\
\hline Achyranthes aspera L. var.1 & Amaranthaceae & $\mathrm{N}$ & native & wide \\
\hline Achyranthes aspera L. var. 2 & Amaranthaceae & $\mathrm{N}$ & native & local \\
\hline Acrostichum aureum $\mathrm{L}$. & Pteridaceae & $\mathrm{N}$ & native & local \\
\hline Adansonia digitata $\mathrm{L}$. & Malvaceae & $A$ & not naturalized, cultivated & supposed extinct \\
\hline Agave sisalana Perrine & Asparagaceae & A & naturalized, cultivated & local \\
\hline Albizia lebbeck (L.) Benth. & Fabaceae & A & not naturalized, cultivated & local \\
\hline Amaranthus graecizans L. subsp. silvestris (Vill.) Brenan & Amaranthaceae & A & naturalized & local \\
\hline Amaranthus viridis $L$. & Amaranthaceae & $A$ & naturalized & local \\
\hline Antigonon leptopus Hook. \& Arn. & Polygonaceae & A & naturalized, cultivated & local \\
\hline Asystasia sp. & Acanthaceae & $\mathrm{N}$ & native & local \\
\hline Bambusa vulgaris Schrad. ex J.C. Wendl. var. vittata Rivière \& C. Rivière & Poaceae & A & not naturalized, cultivated & local \\
\hline Boerhavia coccinea Mill. & Nyctaginaceae & $\mathrm{N}$ & native & wide \\
\hline Boerhavia erecta $\mathrm{L}$. & Nyctaginaceae & A & naturalized & local \\
\hline Boerhavia sp. nov. 1 ? & Nyctaginaceae & $\mathrm{N}$ & native & wide \\
\hline Bougainvillea $x$ buttiana Holttum \& Standl. & Nyctaginaceae & A & not naturalized, cultivated & local \\
\hline Bulbostylis hispidula (Vahl) R.W. Haines subsp. hispidula & Cyperaceae & $\mathrm{N}$ & native & local \\
\hline Calophyllum inophyllum L. & Calophyllaceae & $A$ & not naturalized, cultivated & local \\
\hline Canavalia rosea (Sw.) DC. & Fabaceae & $\mathrm{N}$ & native & local \\
\hline Capparis cartilaginea Decne. & Capparaceae & $\mathrm{N}$ & native & local \\
\hline Cascabela thevetia (L.) Lippold & Apocynaceae & $A$ & not naturalized, cultivated & local \\
\hline Cassytha filiformis $\mathrm{L}$. & Lauraceae & $\mathrm{N}$ & native & wide \\
\hline Casuarina equisetifolia $\mathrm{L}$. & Casuarinaceae & $A$ & invasive, cultivated & wide \\
\hline Catharanthus roseus (L.) G. Don & Apocynaceae & $A$ & naturalized, cultivated & local \\
\hline Ceiba pentandra (L.) Gaertn. & Malvaceae & $A$ & not naturalized, cultivated & local \\
\hline Celosia spicata (Thouars) Spreng. & Amaranthaceae & $\mathrm{N}$ & native & local \\
\hline Cenchrus echinatus L. & Poaceae & $A$ & naturalized & local \\
\hline Cheirolaena linearis Benth & Malvaceae & $\mathrm{N}$ & native & local \\
\hline
\end{tabular}




\begin{tabular}{|c|c|c|c|c|}
\hline Scientific name & Family & $\begin{array}{l}\text { General status on } \\
\text { Juan de Nova }\end{array}$ & $\begin{array}{c}\text { Population and cultural status on } \\
\text { Juan de Nova }\end{array}$ & $\begin{array}{c}\text { Distribution range } \\
\text { on Juan de Nova }\end{array}$ \\
\hline Citrus aurantiifolia (Christm.) Swingle & Rutaceae & A & not naturalized, cultivated & local \\
\hline Cleome grandidieri Baill. & Cleomaceae & $\mathrm{N}$ & native & local \\
\hline Cleome strigosa (Boj.) Oliv. & Cleomaceae & $\mathrm{N}$ & native & wide \\
\hline Cocos nucifera $\mathrm{L}$. & Arecaceae & $A$ & invasive, cultivated & wide \\
\hline Colubrina asiatica (L.) Brongn. & Rhamnaceae & $\mathrm{N}$ & native & local \\
\hline Commelina forskalaei Vahl & Commelinaceae & $A$ & naturalized & local \\
\hline Commicarpus plumbagineus (Cav.) Standl. & Nyctaginaceae & $\mathrm{N}$ & native & local \\
\hline Consolea falcata (Ekman \& Werderm.) F.M. Knuth & Cactaceae & A & naturalized, cultivated & local \\
\hline Corchorus aestuans L. & Malvaceae & A & naturalized & local \\
\hline Cordia subcordata Lam. & Boraginaceae & $\mathrm{N}$ & native, cultivated & local \\
\hline Crotalaria edmundi-bakeri R. Vig. & Fabaceae & $\mathrm{K}$ & native or naturalized & local \\
\hline Cucumis melo L. subsp. agrestis (Naudin) Pangalo var. agrestis & Cucurbitaceae & K & native or naturalized & local \\
\hline Cynodon dactylon (L.) Pers. & Poaceae & $\mathrm{N}$ & native & local \\
\hline Cynodon sp. & Poaceae & A & naturalized & local \\
\hline Cyperus rotundus $\mathrm{L}$. & Cyperaceae & A & naturalized & local \\
\hline Dactyloctenium aegyptium (L.) Willd. & Poaceae & A & naturalized & local \\
\hline Dactyloctenium ctenioides (Steud.) Lorch ex Bosser & Poaceae & $\mathrm{N}$ & native & wide \\
\hline Dactyloctenium geminatum Hack. & Poaceae & $\mathrm{N}$ & native & local \\
\hline Daknopholis boivinii (A. Camus) Clayton & Poaceae & $\mathrm{N}$ & native & local \\
\hline Datura innoxia Mill. & Solanaceae & $A$ & naturalized & local \\
\hline Delonix regia (Bojer) Raf. & Fabaceae & $A$ & not naturalized, cultivated & local \\
\hline Digitaria setigera Roth & Poaceae & $\mathrm{K}$ & native or naturalized & local \\
\hline Dodonaea viscosa (L.) Jacq. & Sapindaceae & $\mathrm{K}$ & native or naturalized & local \\
\hline Dombeya greveana Baill. var. metameropsis (Hochr.) Arènes & Malvaceae & $\mathrm{N}$ & native, cultivated & wide \\
\hline Eragrostis cilianensis (All.) Vignolo ex Janch. & Poaceae & A & naturalized & local \\
\hline Eragrostis ciliaris (L.) R. Br. & Poaceae & $\mathrm{N}$ & native & wide \\
\hline Eragrostis minor Host & Poaceae & $A$ & naturalized & local \\
\hline Eriochloa fatmensis (Hochst. \& Steud.) Clayton & Poaceae & A & naturalized & local \\
\hline Erythrina variegata $\mathrm{L}$. & Fabaceae & $A$ & not naturalized, cultivated & local \\
\hline Euphorbia hirta L. & Euphorbiaceae & $A$ & naturalized & wide \\
\hline Euphorbia mertonii Fosberg & Euphorbiaceae & $\mathrm{N}$ & native & local \\
\hline Euphorbia prostrata Aiton & Euphorbiaceae & $A$ & naturalized & wide \\
\hline Euphorbia stenoclada Baill. & Euphorbiaceae & $\mathrm{N}$ & native & local \\
\hline
\end{tabular}




\begin{tabular}{|c|c|c|c|c|}
\hline Scientific name & Family & $\begin{array}{l}\text { General status on } \\
\text { Juan de Nova }\end{array}$ & $\begin{array}{c}\text { Population and cultural status on } \\
\text { Juan de Nova }\end{array}$ & $\begin{array}{l}\text { Distribution range } \\
\text { on Juan de Nova }\end{array}$ \\
\hline Euphorbia thymifolia L. & Euphorbiaceae & A & naturalized & local \\
\hline Euphorbia tithymaloides L. & Euphorbiaceae & $A$ & naturalized, cultivated & local \\
\hline Ficus benghalensis $\mathrm{L}$. & Moraceae & $A$ & not naturalized, cultivated & local \\
\hline Furcraea selloa K. Koch var. marginata Trel. & Asparagaceae & A & not naturalized, cultivated & local \\
\hline Gossypium hirsutum L. & Malvaceae & A & invasive, cultivated & wide \\
\hline Guettarda speciosa L. & Rubiaceae & $\mathrm{N}$ & native & local \\
\hline Guilandina bonduc L. & Fabaceae & $\mathrm{N}$ & native & wide \\
\hline Heliotropium foertherianum Diane \& Hilger & Boraginaceae & $\mathrm{N}$ & native & wide \\
\hline Heteropogon contortus (L.) P. Beauv. ex Roem. \& Schult. & Poaceae & $\mathrm{K}$ & native or naturalized & local \\
\hline Hibiscus physaloides Guill. \& Perr. & Malvaceae & $\mathrm{K}$ & native or naturalized & local \\
\hline Hyphaene coriacea Gaertn. & Arecaceae & $\mathrm{K}$ & not naturalized, cultivated & local \\
\hline Hypoestes juanensis Benoist & Acanthaceae & $\mathrm{N}$ & native & wide \\
\hline Imperata cylindrica (L.) P. Beauv. & Poaceae & $A$ & naturalized, cultivated & local \\
\hline Indigofera tinctoria $\mathrm{L}$. & Fabaceae & $A$ & naturalized & local \\
\hline Ipomoea pes-caprae (L.) R. Br. subsp. brasiliensis (L.) Ooststr. & Convolvulaceae & $\mathrm{N}$ & native & local \\
\hline Ipomoea violacea $\mathrm{L}$. & Convolvulaceae & $\mathrm{N}$ & native & wide \\
\hline Lantana strigocamara R.W. Sanders & Verbenaceae & $A$ & invasive & wide \\
\hline Launaea intybacea (Jacq.) Beauverd & Asteraceae & $\mathrm{N}$ & native & local \\
\hline Launaea sarmentosa (Willd.) Schultz & Asteraceae & $\mathrm{N}$ & native & local \\
\hline Leptadenia madagascariensis Decne. & Apocynaceae & $\mathrm{N}$ & native & wide \\
\hline Lepturus repens (G. Forst.) R. Br. & Poaceae & $\mathrm{N}$ & native & local \\
\hline Lumnitzera racemosa Willd. & Combretaceae & $\mathrm{N}$ & native & local \\
\hline Maerua baillonii Hadj-Moust. & Capparaceae & $\mathrm{N}$ & native & local \\
\hline Manihot esculenta Crantz & Euphorbiaceae & $A$ & not naturalized, cultivated & supposed extinct \\
\hline Marsdenia verrucosa Decne. & Apocynaceae & $\mathrm{K}$ & native or naturalized & wide \\
\hline Mimusops coriacea (A. DC.) Miq. & Sapotaceae & $A$ & not naturalized, cultivated & local \\
\hline Mollugo nudicaulis Lam. & Molluginaceae & $\mathrm{N}$ & native & local \\
\hline Moringa oleifera Lam. & Moringaceae & A & not naturalized, cultivated & local \\
\hline Morus alba L. & Moraceae & $A$ & not naturalized, cultivated & local \\
\hline Mucuna gigantea (Willd.) DC. & Fabaceae & $\mathrm{N}$ & native & local \\
\hline Musa sp. & Musaceae & $A$ & not naturalized, cultivated & supposed extinct \\
\hline Nervilia bicarinata (Blume) Schltr. & Orchidaceae & $\mathrm{N}$ & native & local \\
\hline Nesogenes madagascariensis (Bonati) Marais & Orobanchaceae & $\mathrm{N}$ & native & local \\
\hline
\end{tabular}




\begin{tabular}{|c|c|c|c|c|}
\hline Scientific name & Family & $\begin{array}{l}\text { General status on } \\
\text { Juan de Nova }\end{array}$ & $\begin{array}{c}\text { Population and cultural status on } \\
\text { Juan de Nova }\end{array}$ & $\begin{array}{l}\text { Distribution range } \\
\text { on Juan de Nova }\end{array}$ \\
\hline Oldenlandia corymbosa L. var. caespitosa (Benth.) Verdc. & Rubiaceae & $\bar{A}$ & naturalized & wide \\
\hline Oldenlandia fastigiata Bremek. var. fastigiata & Rubiaceae & $\mathrm{K}$ & native or naturalized & local \\
\hline Oldenlandia fastigiata Bremek. var. somala (Bremek.) Verdc. & Rubiaceae & $\mathrm{K}$ & native or naturalized & local \\
\hline Ophioglossum lancifolium C. Presl & Ophioglossaceae & $\mathrm{N}$ & native & local \\
\hline Opuntia stricta (Haw.) Haw. & Cactaceae & A & not naturalized, cultivated & local \\
\hline Panicum cf. voeltzkowii Mez & Poaceae & $\mathrm{K}$ & native or naturalized & wide \\
\hline Pemphis acidula J.R. Forst. \& G. Forst. & Lythraceae & $\mathrm{N}$ & native & local \\
\hline Perrierophytum glomeratum Hochr. & Malvaceae & $\mathrm{N}$ & native & local \\
\hline Phyllanthus amarus Schumach. & Phyllanthaceae & $A$ & naturalized & local \\
\hline Phyllanthus coluteoides Baill. ex Müll.Arg. & Phyllanthaceae & $\mathrm{K}$ & native or naturalized, cultivated & wide \\
\hline Phyllanthus maderaspatensis L. s. I. & Phyllanthaceae & $\mathrm{N}$ & native & local \\
\hline Physalis lagascae Roem. \& Schult. & Solanaceae & A & naturalized & local \\
\hline Pisonia grandis $\mathrm{R}$. Br. & Nyctaginaceae & $\mathrm{N}$ & native & wide \\
\hline Pithecellobium dulce (Roxb.) Benth. & Fabaceae & $A$ & naturalized, cultivated & local \\
\hline Pleurostelma cernuum (Decne.) Bullock & Apocynaceae & $\mathrm{N}$ & native & wide \\
\hline Plumeria rubra L. f. tricolor (Ruiz \& Pav.) Woodson & Apocynaceae & A & not naturalized, cultivated & local \\
\hline Pongamia pinnata (L.) Pierre & Fabaceae & A & not naturalized, cultivated & local \\
\hline Portulaca granulatostellulata (Poelln.) Ricceri \& Arrigoni & Portulacaceae & K & native or naturalized & local \\
\hline Portulaca nitida (Danin et H.G. Baker) Ricceri et Arrigoni & Portulacaceae & $\mathrm{N}$ & native & local \\
\hline $\begin{array}{l}\text { Pseuderanthemum carruthersii (Seem.) Guill. var. atropurpureum (W. Bull) } \\
\text { Fosberg }\end{array}$ & Acanthaceae & A & not naturalized, cultivated & local \\
\hline Pseudoconyza viscosa (Mill.) D'Arcy & Asteraceae & K & native or naturalized & local \\
\hline Psiadia altissima (DC.) Drake & Asteraceae & $\mathrm{N}$ & native, cultivated & wide \\
\hline Rhizophora mucronata Lam. & Rhizophoraceae & $\mathrm{N}$ & native & local \\
\hline Ricinus communis $\mathrm{L}$. & Euphorbiaceae & A & naturalized & local \\
\hline Salvadora angustifolia Turrill & Salvadoraceae & $\mathrm{N}$ & native, cultivated & wide \\
\hline Scaevola taccada (Gaertn.) Roxb. & Goodeniaceae & $\mathrm{N}$ & native & wide \\
\hline Sclerodactylon macrostachyum (Benth.) A. Camus & Poaceae & $\mathrm{N}$ & native & wide \\
\hline Senna occidentalis (L.) Link & Fabaceae & A & naturalized & local \\
\hline Senna sp. & Fabaceae & $A$ & naturalized, cultivated & local \\
\hline Sida pusilla Cav. s. I. & Malvaceae & A & naturalized & wide \\
\hline Sideroxylon inerme L. & Sapotaceae & $\mathrm{N}$ & native & local \\
\hline Solanum americanum Mill. & Solanaceae & $A$ & naturalized & local \\
\hline
\end{tabular}




\begin{tabular}{|c|c|c|c|c|}
\hline Scientific name & Family & $\begin{array}{l}\text { General status on } \\
\text { Juan de Nova }\end{array}$ & $\begin{array}{l}\text { Population and cultural status on } \\
\text { Juan de Nova }\end{array}$ & $\begin{array}{l}\text { Distribution range } \\
\text { on Juan de Nova }\end{array}$ \\
\hline Sporobolus virginicus (L.) Kunth & Poaceae & $\mathrm{N}$ & native & wide \\
\hline Suriana maritima $\mathrm{L}$. & Surianaceae & $\mathrm{N}$ & native & wide \\
\hline Tabebuia pallida (Lindl.) Miers & Bignoniaceae & A & not naturalized, cultivated & local \\
\hline Tamarindus indica $\mathrm{L}$. & Fabaceae & A & naturalized, cultivated & local \\
\hline Tephrosia purpurea (L.) Pers. subsp. dunensis Brummitt & Fabaceae & $\mathrm{N}$ & native & local \\
\hline Terminalia catappa $\mathrm{L}$. & Combretaceae & A & not naturalized, cultivated & local \\
\hline Thespesia populneoides (Roxb.) Kostel. & Malvaceae & $\mathrm{N}$ & native, cultivated & local \\
\hline Tradescantia spathacea Sw. & Commelinaceae & A & not naturalized, cultivated & local \\
\hline Trianthema portulacastrum L. & Aizoaceae & $A$ & naturalized & local \\
\hline Tribulus cistoides L. & Zygophyllaceae & A & naturalized & local \\
\hline Tricholaena monachne (Trin.) Stapf \& C.E. Hubb. & Poaceae & K & native or naturalized & local \\
\hline Tridax procumbens $\mathrm{L}$. & Asteraceae & A & naturalized & wide \\
\hline Urochloa deflexa (Schumach.) H. Scholz & Poaceae & $\mathrm{K}$ & native or naturalized & local \\
\hline Urochloa maxima (Jacq.) R.D. Webster & Poaceae & A & naturalized & local \\
\hline Vachellia farnesiana (L.) Wight \& Arn. & Fabaceae & $A$ & naturalized, cultivated & local \\
\hline Vigna marina (Burm.) Merr. & Fabaceae & $\mathrm{N}$ & native & local \\
\hline Wollastonia biflora (L.) DC. & Asteraceae & $\mathrm{N}$ & native & wide \\
\hline Zea mays L. & Poaceae & $A$ & not naturalized, cultivated & supposed extinct \\
\hline
\end{tabular}


Table 5. List of taxa found on the Iles Glorieuses. In the column 'General status on Europa' differents codes were used : $A=$ alien ; N = native ; K = cryptogenic.

\begin{tabular}{|c|c|c|c|c|c|c|c|c|}
\hline Scientific name & Family & $\begin{array}{l}\text { General } \\
\text { status on Iles } \\
\text { Glorieuses }\end{array}$ & $\begin{array}{c}\text { Population and cultural } \\
\text { status on lles } \\
\text { Glorieuses }\end{array}$ & $\begin{array}{l}\text { Distribution } \\
\text { range on lles } \\
\text { Glorieuses }\end{array}$ & $\begin{array}{l}\text { Presence } \\
\text { on Grande } \\
\text { Glorieuse }\end{array}$ & $\begin{array}{l}\text { Presence } \\
\text { on lle aux } \\
\text { Crabes }\end{array}$ & $\begin{array}{l}\text { Presence } \\
\text { on lle du } \\
\text { Lys }\end{array}$ & $\begin{array}{l}\text { Presence on } \\
\text { Ile aux } \\
\text { Roches } \\
\text { Vertes }\end{array}$ \\
\hline Acalypha indica L. & Euphorbiaceae & A & naturalized & local & $\mathrm{x}$ & & & \\
\hline Achyranthes aspera L. var. fruticosa (Lam.) Boerl. & Amaranthaceae & $\mathrm{N}$ & native & wide & $\mathrm{x}$ & $\mathrm{x}$ & $\mathrm{x}$ & \\
\hline Adiantum hirsutum Bory & Pteridaceae & $\mathrm{N}$ & native & local & $x$ & & & \\
\hline Adiantum philippense L. & Pteridaceae & $\mathrm{N}$ & native & local & $x$ & & & \\
\hline Agave sisalana Perrine & Asparagaceae & A & invasive, cultivated & local & $x$ & & & \\
\hline Ageratum conyzoides $\mathrm{L}$. & Asteraceae & A & naturalized & local & $x$ & & & \\
\hline Alocasia macrorrhizos (L.) G. Don var. macrorrhizos & Araceae & A & naturalized, cultivated & local & $\mathrm{x}$ & & & \\
\hline Amaranthus spinosus L. & Amaranthaceae & A & naturalized & local & $\mathrm{x}$ & & & \\
\hline Amaranthus viridis $\mathrm{L}$. & Amaranthaceae & A & naturalized & local & $x$ & & & \\
\hline Annona muricata $\mathrm{L}$. & Annonaceae & A & naturalized, cultivated & local & $\mathrm{x}$ & & & \\
\hline Annona squamosa $\mathrm{L}$. & Annonaceae & $A$ & naturalized, cultivated & local & $x$ & & & \\
\hline Asystasia sp. & Acanthaceae & $\mathrm{N}$ & native & local & $\mathrm{x}$ & & & \\
\hline Boerhavia diffusa L. & Nyctaginaceae & A & naturalized & local & $x$ & & & \\
\hline Boerhavia erecta $\mathrm{L}$. & Nyctaginaceae & A & naturalized & local & $\mathrm{x}$ & & & \\
\hline Boerhavia sp. nov. 1 ? & Nyctaginaceae & $\mathrm{N}$ & native & wide & $x$ & & $\mathrm{x}$ & \\
\hline Bothriochloa pertusa (L.) A. Camus & Poaceae & $A$ & naturalized & local & $x$ & & & \\
\hline Bulbostylis basalis Fosberg & Cyperaceae & $\mathrm{N}$ & native & local & $x$ & & & \\
\hline Calophyllum inophyllum L. & Calophyllaceae & $\mathrm{N}$ & native & local & $x$ & & & \\
\hline Canavalia rosea (Sw.) DC. & Fabaceae & $\mathrm{N}$ & native & local & $x$ & & & \\
\hline Capsicum frutescens $\mathrm{L}$. & Solanaceae & $A$ & naturalized, cultivated & local & $x$ & & & \\
\hline Carica papaya L. & Caricaceae & A & invasive, cultivated & local & $x$ & & & \\
\hline Cassytha filiformis L. & Lauraceae & $\mathrm{N}$ & native & wide & $x$ & & & \\
\hline Casuarina equisetifolia $\mathrm{L}$. & Casuarinaceae & A & invasive, cultivated & wide & $x$ & & & \\
\hline Catharanthus roseus (L.) G. Don & Apocynaceae & $A$ & naturalized & local & $x$ & & & \\
\hline Celosia spicata (Thouars) Spreng. & Amaranthaceae & $\mathrm{N}$ & native & local & $x$ & & & \\
\hline Cenchrus echinatus L. & Poaceae & A & naturalized & local & $x$ & & & \\
\hline Cenchrus polystachios (L.) Morrone & Poaceae & $A$ & invasive & local & $x$ & & & \\
\hline Chloris barbata Sw. & Poaceae & A & naturalized & local & $x$ & & & \\
\hline
\end{tabular}




\begin{tabular}{|c|c|c|c|c|c|c|c|c|}
\hline Scientific name & Family & $\begin{array}{c}\text { General } \\
\text { status on Iles } \\
\text { Glorieuses }\end{array}$ & $\begin{array}{l}\text { Population and cultural } \\
\text { status on lles } \\
\text { Glorieuses }\end{array}$ & $\begin{array}{c}\text { Distribution } \\
\text { range on Iles } \\
\text { Glorieuses }\end{array}$ & $\begin{array}{l}\text { Presence } \\
\text { on Grande } \\
\text { Glorieuse }\end{array}$ & $\begin{array}{c}\text { Presence } \\
\text { on lle aux } \\
\text { Crabes }\end{array}$ & $\begin{array}{l}\text { Presence } \\
\text { on lle du } \\
\text { Lys }\end{array}$ & $\begin{array}{l}\text { Presence on } \\
\text { Ile aux } \\
\text { Roches } \\
\text { Vertes }\end{array}$ \\
\hline Christella cf. dentata (Forssk.) Brownsey \& Jermy & Thelypteridaceae & A & naturalized & $\begin{array}{l}\text { supposed } \\
\text { extinct }\end{array}$ & $\mathrm{x}$ & & & \\
\hline Cleome strigosa (Boj.) Oliv. & Cleomaceae & $\mathrm{N}$ & native & local & $x$ & & & \\
\hline Cocos nucifera $\mathrm{L}$. & Arecaceae & $A$ & invasive, cultivated & wide & $x$ & $x$ & $x$ & \\
\hline Colubrina asiatica (L.) Brongn. & Rhamnaceae & $\mathrm{N}$ & native & wide & $x$ & & & \\
\hline Commelina benghalensis $\mathrm{L}$. & Commelinaceae & $A$ & naturalized & local & $x$ & & & \\
\hline Commicarpus plumbagineus (Cav.) Standl. & Nyctaginaceae & $\mathrm{N}$ & native & local & $x$ & & & \\
\hline Corchorus aestuans L. & Malvaceae & $A$ & naturalized & local & $x$ & & & \\
\hline Cordia subcordata Lam. & Boraginaceae & $\mathrm{N}$ & native & wide & $x$ & & $x$ & \\
\hline $\begin{array}{l}\text { Cucumis melo L. subsp. agrestis (Naudin) Pangalo } \\
\text { var. agrestis }\end{array}$ & Cucurbitaceae & $A$ & naturalized & local & $x$ & & & \\
\hline Cyanthillium cinereum (L.) H. Rob. & Asteraceae & $A$ & naturalized & wide & $x$ & & & \\
\hline Cyperus dubius Rottb. & Cyperaceae & $\mathrm{K}$ & native or naturalized & local & $x$ & & & \\
\hline Cyperus ligularis L. & Cyperaceae & $\mathrm{N}$ & native & local & $x$ & & $x$ & \\
\hline Cyperus rotundus L. & Cyperaceae & $A$ & naturalized & local & $x$ & & & \\
\hline Dactyloctenium ctenioides (Steud.) Lorch ex Bosser & Poaceae & $\mathrm{N}$ & native & wide & $x$ & & $x$ & \\
\hline Daknopholis boivinii (A. Camus) Clayton & Poaceae & $\mathrm{N}$ & native & wide & $x$ & & & \\
\hline Datura innoxia Mill. & Solanaceae & $A$ & naturalized & local & $x$ & & & \\
\hline Digitaria horizontalis Willd. & Poaceae & $A$ & naturalized & local & $x$ & & & \\
\hline Digitaria sp. & Poaceae & $A$ & naturalized & local & $x$ & & & \\
\hline Disperis tripetaloides (Thouars) Lindl. & Orchidaceae & $\mathrm{N}$ & native & local & $x$ & & & \\
\hline Dodonaea viscosa (L.) Jacq. & Sapindaceae & $\mathrm{N}$ & naturalized & $\begin{array}{l}\text { supposed } \\
\text { extinct }\end{array}$ & $x$ & & & \\
\hline Eleusine indica (L.) Gaertn. & Poaceae & $A$ & naturalized & local & $x$ & & & \\
\hline $\begin{array}{l}\text { Enteropogon sechellensis (Baker) Benth. ex T. } \\
\text { Durand \& Schinz }\end{array}$ & Poaceae & $\mathrm{K}$ & native or naturalized & local & $x$ & & & \\
\hline Eragrostis ciliaris (L.) R. Br. & Poaceae & $\mathrm{K}$ & native or naturalized & wide & $x$ & & & \\
\hline Eragrostis subaequiglumis Renvoize & Poaceae & $\mathrm{N}$ & native & local & $x$ & & $x$ & \\
\hline Erythrina variegata L. & Fabaceae & $\mathrm{N}$ & native & $\begin{array}{l}\text { supposed } \\
\text { extinct }\end{array}$ & $x$ & & & \\
\hline Euphorbia hirta L. & Euphorbiaceae & $A$ & naturalized & wide & $x$ & & & \\
\hline
\end{tabular}




\begin{tabular}{|c|c|c|c|c|c|c|c|c|}
\hline Scientific name & Family & $\begin{array}{c}\text { General } \\
\text { status on Iles } \\
\text { Glorieuses }\end{array}$ & $\begin{array}{c}\text { Population and cultural } \\
\text { status on lles } \\
\text { Glorieuses }\end{array}$ & $\begin{array}{l}\text { Distribution } \\
\text { range on Iles } \\
\text { Glorieuses }\end{array}$ & $\begin{array}{l}\text { Presence } \\
\text { on Grande } \\
\text { Glorieuse }\end{array}$ & $\begin{array}{l}\text { Presence } \\
\text { on lle aux } \\
\text { Crabes }\end{array}$ & $\begin{array}{l}\text { Presence } \\
\text { on Ile du } \\
\text { Lys }\end{array}$ & $\begin{array}{l}\text { Presence on } \\
\text { Ile aux } \\
\text { Roches } \\
\text { Vertes }\end{array}$ \\
\hline Euphorbia prostrata Aiton & Euphorbiaceae & $A$ & naturalized & local & $x$ & & & \\
\hline Euphorbia stoddartii Fosberg & Euphorbiaceae & $\mathrm{N}$ & native & wide & $x$ & & $x$ & $x$ \\
\hline Euphorbia tithymaloides L. & Euphorbiaceae & $A$ & naturalized, cultivated & local & $x$ & & & \\
\hline Evolvulus alsinoides (L.) L. & Convolvulaceae & $\mathrm{N}$ & native & local & $x$ & & & \\
\hline Ficus grevei Baillon & Moraceae & $\mathrm{N}$ & native & wide & $x$ & & & \\
\hline Ficus sp. & Moraceae & $\mathrm{K}$ & native or naturalized & local & $x$ & & & \\
\hline Fimbristylis cymosa R. Br. s. I. & Cyperaceae & $\mathrm{N}$ & native & wide & $x$ & & & \\
\hline Flacourtia indica (Burm. f.) Merr. & Salicaceae & $A$ & invasive & wide & $x$ & & & \\
\hline Flueggea virosa (Roxb. ex Willd.) Royle & Phyllanthaceae & $\mathrm{K}$ & native or naturalized & wide & $x$ & & & \\
\hline Furcraea foetida (L.) Haw. & Asparagaceae & $A$ & naturalized, cultivated & local & $x$ & & & \\
\hline Gossypium hirsutum L. & Malvaceae & $A$ & naturalized, cultivated & local & $x$ & & & \\
\hline Guettarda speciosa L. & Rubiaceae & $\mathrm{N}$ & native & wide & $x$ & & $x$ & \\
\hline Guilandina bonduc L. & Fabaceae & $\mathrm{N}$ & native & wide & $x$ & & $x$ & \\
\hline Gynandropsis gynandra (L.) Briq. & Cleomaceae & $\mathrm{K}$ & native or naturalized & local & & & $x$ & \\
\hline Heliotropium foertherianum Diane \& Hilger & Boraginaceae & $\mathrm{N}$ & native & wide & $x$ & & $x$ & \\
\hline Hernandia nymphaeifolia (C. Presl) Kubitzki & Hernandiaceae & $\mathrm{N}$ & native & local & $x$ & & & \\
\hline $\begin{array}{l}\text { Heteropogon contortus (L.) P. Beauv. ex Roem. \& } \\
\text { Schult. }\end{array}$ & Poaceae & A & naturalized & local & $x$ & & & \\
\hline Hibiscus physaloides Guill. \& Perr. & Malvaceae & $\mathrm{N}$ & native & local & $x$ & & $x$ & \\
\hline Hibiscus tiliaceus L. & Malvaceae & $\mathrm{N}$ & native & local & $x$ & & $x$ & \\
\hline Imperata cylindrica (L.) P. Beauv. & Poaceae & A & naturalized, cultivated & local & $x$ & & & \\
\hline $\begin{array}{l}\text { Ipomoea pes-caprae (L.) R. Br. subsp. brasiliensis } \\
\text { (L.) Ooststr. }\end{array}$ & Convolvulaceae & $\mathrm{N}$ & native & wide & $x$ & & $\mathrm{x}$ & \\
\hline Ipomoea violacea $\mathrm{L}$. & Convolvulaceae & $\mathrm{N}$ & native & wide & $x$ & & $\mathrm{x}$ & \\
\hline Launaea intybacea (Jacq.) Beauverd & Asteraceae & $A$ & naturalized & local & $x$ & & & \\
\hline Launaea sarmentosa (Willd.) Schultz & Asteraceae & $\mathrm{N}$ & native & wide & $x$ & & & \\
\hline Lepidium englerianum (Muschl.) Al-Shehbaz & Brassicaceae & $A$ & naturalized & local & $x$ & & & \\
\hline Lepturus repens (G. Forst.) R. Br. & Poaceae & $\mathrm{N}$ & native & local & $x$ & & & \\
\hline Leucaena leucocephala (Lam.) de Wit & Fabaceae & A & naturalized, cultivated & local & $x$ & & & \\
\hline Lygodium kerstenii Kuhn & Lygodiaceae & $\mathrm{N}$ & native & local & $x$ & & & \\
\hline Malvastrum coromandelianum (L.) Garcke & Malvaceae & A & naturalized & local & $x$ & & & \\
\hline
\end{tabular}




\begin{tabular}{|c|c|c|c|c|c|c|c|c|}
\hline Scientific name & Family & $\begin{array}{l}\text { General } \\
\text { status on Iles } \\
\text { Glorieuses }\end{array}$ & $\begin{array}{c}\text { Population and cultural } \\
\text { status on lles } \\
\text { Glorieuses }\end{array}$ & $\begin{array}{l}\text { Distribution } \\
\text { range on lles } \\
\text { Glorieuses }\end{array}$ & $\begin{array}{l}\text { Presence } \\
\text { on Grande } \\
\text { Glorieuse }\end{array}$ & $\begin{array}{c}\text { Presence } \\
\text { on Ile aux } \\
\text { Crabes }\end{array}$ & $\begin{array}{l}\text { Presence } \\
\text { on Ile du } \\
\text { Lys }\end{array}$ & $\begin{array}{l}\text { Presence on } \\
\text { Ile aux } \\
\text { Roches } \\
\text { Vertes }\end{array}$ \\
\hline Mollugo nudicaulis Lam. & Molluginaceae & $\mathrm{N}$ & native & wide & $\mathrm{x}$ & & & \\
\hline Morinda citrifolia $L$. & Rubiaceae & $\mathrm{N}$ & native & local & $x$ & & & \\
\hline Moringa oleifera Lam. & Moringaceae & $A$ & naturalized, cultivated & local & $x$ & & & \\
\hline Mucuna gigantea (Willd.) DC. & Fabaceae & $\mathrm{N}$ & native & local & $x$ & & & \\
\hline Musa sp. & Musaceae & $A$ & $\begin{array}{l}\text { not naturalized, } \\
\text { cultivated }\end{array}$ & $\begin{array}{l}\text { supposed } \\
\text { extinct }\end{array}$ & $x$ & & & \\
\hline Nephrolepis biserrata (Sw.) Schott & Nephrolepidaceae & $\mathrm{N}$ & native & local & $x$ & & & \\
\hline Nervilia bicarinata (Blume) Schltr. & Orchidaceae & $\mathrm{N}$ & native & local & $x$ & & & \\
\hline Nesogenes prostrata (Benth.) Hemsl. & Orobanchaceae & $\mathrm{N}$ & native & local & $x$ & & & \\
\hline Ochrosia oppositifolia (Lam.) K. Schum. & Apocynaceae & $\mathrm{N}$ & native & local & $\mathrm{x}$ & & & \\
\hline Oldenlandia corymbosa L. var. corymbosa & Rubiaceae & $A$ & naturalized & local & $x$ & & & \\
\hline Panicum voeltzkowii Mez & Poaceae & $\mathrm{N}$ & native & local & $x$ & & & \\
\hline Paspalum vaginatum Sw. & Poaceae & $\mathrm{N}$ & native & local & & & $x$ & \\
\hline Passiflora suberosa L. & Passifloraceae & $A$ & invasive & wide & $x$ & & & \\
\hline Pemphis acidula J.R. Forst. \& G. Forst. & Lythraceae & $\mathrm{N}$ & native & local & $x$ & & $x$ & \\
\hline Perrierophytum glomeratum Hochr. & Malvaceae & $\mathrm{N}$ & native & wide & $x$ & & & \\
\hline Phyllanthus amarus Schumach. & Phyllanthaceae & $A$ & naturalized & local & $x$ & & & \\
\hline Phyllanthus maderaspatensis L. s. I. & Phyllanthaceae & $\mathrm{N}$ & native & wide & $x$ & & & \\
\hline Phymatosorus scolopendria (Burm. f.) Pic. Serm. & Polypodiaceae & $\mathrm{N}$ & native & local & $x$ & & & \\
\hline Physalis lagascae Roem. \& Schult. & Solanaceae & A & naturalized & local & $x$ & & & \\
\hline Pisonia grandis $\mathrm{R} . \mathrm{Br}$. & Nyctaginaceae & $\mathrm{N}$ & native & wide & $x$ & & $x$ & \\
\hline $\begin{array}{l}\text { Portulaca granulatostellulata (Poelln.) Ricceri \& } \\
\text { Arrigoni }\end{array}$ & Portulacaceae & $\mathrm{K}$ & native or naturalized & local & $\mathrm{x}$ & $x$ & $\mathrm{x}$ & \\
\hline $\begin{array}{l}\text { Portulaca mauritiensis Poelln. var. aldabrensis } \\
\text { Fosberg }\end{array}$ & Portulacaceae & $\mathrm{N}$ & native & local & $x$ & $x$ & & \\
\hline $\begin{array}{l}\text { Portulaca nitida (Danin et H.G. Baker) Ricceri et } \\
\text { Arrigoni }\end{array}$ & Portulacaceae & $\mathrm{N}$ & native & local & $x$ & & $x$ & $x$ \\
\hline Premna serratifolia L. & Lamiaceae & $\mathrm{N}$ & native & local & $x$ & & & \\
\hline Pseudoconyza viscosa (Mill.) D'Arcy & Asteraceae & $A$ & naturalized & local & $x$ & & $x$ & \\
\hline Psilotum nudum (L.) P. Beauv. & Psilotaceae & $\mathrm{N}$ & native & local & $x$ & & & \\
\hline
\end{tabular}




\begin{tabular}{|c|c|c|c|c|c|c|c|c|}
\hline Scientific name & Family & $\begin{array}{c}\text { General } \\
\text { status on Iles } \\
\text { Glorieuses }\end{array}$ & $\begin{array}{c}\text { Population and cultural } \\
\text { status on Iles } \\
\text { Glorieuses }\end{array}$ & $\begin{array}{l}\text { Distribution } \\
\text { range on lles } \\
\text { Glorieuses }\end{array}$ & $\begin{array}{l}\text { Presence } \\
\text { on Grande } \\
\text { Glorieuse }\end{array}$ & $\begin{array}{l}\text { Presence } \\
\text { on Ile aux } \\
\text { Crabes }\end{array}$ & $\begin{array}{l}\text { Presence } \\
\text { on lle du } \\
\text { Lys }\end{array}$ & $\begin{array}{l}\text { Presence on } \\
\text { lle aux } \\
\text { Roches } \\
\text { Vertes }\end{array}$ \\
\hline Pteris linearis Poir. & Pteridaceae & A & naturalized & $\begin{array}{l}\text { supposed } \\
\text { extinct }\end{array}$ & $\mathrm{x}$ & & & \\
\hline Ricinus communis $\mathrm{L}$. & Euphorbiaceae & $A$ & naturalized & local & $x$ & & & \\
\hline Scaevola taccada (Gaertn.) Roxb. & Goodeniaceae & $\mathrm{N}$ & native & wide & $x$ & & $x$ & \\
\hline Sclerodactylon macrostachyum (Benth.) A. Camus & Poaceae & $\mathrm{N}$ & native & local & $x$ & & $\mathrm{x}$ & \\
\hline Secamone pachystigma Jum. \& H. Perrier & Apocynaceae & $\mathrm{N}$ & native & local & $x$ & & & \\
\hline Senna occidentalis (L.) Link & Fabaceae & $A$ & naturalized & local & $x$ & & & \\
\hline Sesuvium portulacastrum (L.) L. & Aizoaceae & $\mathrm{N}$ & native & local & & $x$ & & $x$ \\
\hline Sida acuta Burm. f. & Malvaceae & $A$ & naturalized & local & $x$ & & & \\
\hline Sida pusilla Cav. s. I. & Malvaceae & $\mathrm{N}$ & native & wide & $x$ & & $x$ & \\
\hline Sida rhombifolia $\mathrm{L}$. & Malvaceae & $A$ & naturalized & local & $x$ & & & \\
\hline Solanum americanum Mill. & Solanaceae & $A$ & naturalized & local & $x$ & & & \\
\hline Solanum lycopersicum L. & Solanaceae & $A$ & naturalized, cultivated & local & $x$ & & & \\
\hline Solanum sp. & Solanaceae & $A$ & naturalized & local & & & $x$ & \\
\hline Sophora tomentosa L. subsp. tomentosa & Fabaceae & $\mathrm{N}$ & native & local & $x$ & & & \\
\hline Sporobolus virginicus (L.) Kunth & Poaceae & $\mathrm{N}$ & native & wide & $x$ & & & $x$ \\
\hline Stachytarpheta jamaicensis (L.) Vahl & Verbenaceae & $A$ & naturalized & wide & $x$ & & & \\
\hline Stachytarpheta urticifolia Sims & Verbenaceae & $A$ & naturalized & local & $x$ & & & \\
\hline Stenotaphrum micranthum (Desv.) C.E. Hubb., & Poaceae & $\mathrm{K}$ & native or naturalized & local & $x$ & & & \\
\hline Striga asiatica (L.) Kuntze & Orobanchaceae & $\mathrm{N}$ & native & local & $x$ & & & \\
\hline Suriana maritima L. & Surianaceae & $\mathrm{N}$ & native & wide & $x$ & $x$ & & \\
\hline Terminalia catappa $\mathrm{L}$. & Combretaceae & $\mathrm{N}$ & native & local & $x$ & & & \\
\hline Thespesia populneoides (Roxb.) Kostel. & Malvaceae & $\mathrm{N}$ & native & local & $x$ & & $x$ & \\
\hline Tribulus cistoides L. & Zygophyllaceae & $\mathrm{K}$ & native or naturalized & local & $x$ & & & \\
\hline Tridax procumbens L. & Asteraceae & $A$ & naturalized & wide & $x$ & & & \\
\hline Urochloa maxima (Jacq.) R.D. Webster & Poaceae & $A$ & naturalized & local & $x$ & & & \\
\hline Vigna marina (Burm.) Merr. & Fabaceae & $\mathrm{N}$ & native & local & $x$ & & & \\
\hline Wollastonia biflora (L.) DC. & Asteraceae & $\mathrm{N}$ & native & local & $x$ & & & \\
\hline Zea mays $\mathrm{L}$. & Poaceae & A & $\begin{array}{l}\text { not naturalized, } \\
\text { cultivated }\end{array}$ & $\begin{array}{l}\text { supposed } \\
\text { extinct }\end{array}$ & $x$ & & & \\
\hline
\end{tabular}


Table 6. List of taxa found on Tromelin. In the column 'General status on Europa' differents codes were used : A = alien ; $\mathrm{N}=$ native ; $\mathrm{K}=$ cryptogenic.

\begin{tabular}{|c|c|c|c|c|}
\hline Scientific name & Family & $\begin{array}{l}\text { General status on } \\
\text { Tromelin }\end{array}$ & $\begin{array}{c}\text { Population and cultural status on } \\
\text { Tromelin }\end{array}$ & $\begin{array}{c}\text { Distribution range } \\
\text { on Tromelin }\end{array}$ \\
\hline Aloe vera (L.) Burm. f. & Xanthorrhoeaceae & A & naturalized, cultivated & supposed extinct \\
\hline Boerhavia sp. nov. 2 ? & Nyctaginaceae & $\mathrm{N}$ & native & wide \\
\hline Carica papaya L. & Caricaceae & A & not naturalized, cultivated & supposed extinct \\
\hline Casuarina equisetifolia $\mathrm{L}$. & Casuarinaceae & A & not naturalized, cultivated & supposed extinct \\
\hline Cocos nucifera $\mathrm{L}$. & Arecaceae & A & invasive, cultivated & local \\
\hline Consolea falcata (Ekman \& Werderm.) F.M. Knuth & Cactaceae & A & invasive, cultivated & local \\
\hline Dactyloctenium aristatum Link & Poaceae & A & naturalized & local \\
\hline Euphorbia hirta L. & Euphorbiaceae & A & invasive & local \\
\hline Euphorbia prostrata Aiton & Euphorbiaceae & A & invasive & local \\
\hline Heliotropium foertherianum Diane \& Hilger & Boraginaceae & $\mathrm{N}$ & native & wide \\
\hline Ipomoea pes-caprae (L.) R. Br. subsp. brasiliensis (L.) Ooststr. & Convolvulaceae & $\mathrm{N}$ & native & supposed extinct \\
\hline Lepidium englerianum (Muschl.) Al-Shehbaz & Brassicaceae & A & invasive & local \\
\hline Musa sp. & Musaceae & A & not naturalized, cultivated & supposed extinct \\
\hline Pandanus utilis Bory & Pandanaceae & A & not naturalized, cultivated & supposed extinct \\
\hline Persea americana Mill. & Lauraceae & A & not naturalized, cultivated & supposed extinct \\
\hline Portulaca mauritiensis Poelln. var. aldabrensis Fosberg & Portulacaceae & $\mathrm{N}$ & native & local \\
\hline Portulaca gr. oleracea L. & Portulacaceae & $\mathrm{N}$ & native & wide \\
\hline Sida pusilla Cav. s. I. & Malvaceae & $\mathrm{N}$ & native & wide \\
\hline Tamarindus indica $\mathrm{L}$. & Fabaceae & A & not naturalized, cultivated & supposed extinct \\
\hline Terminalia catappa L. & Combretaceae & A & not naturalized, cultivated & local \\
\hline Triumfetta procumbens G. Forst. & Malvaceae & $\mathrm{N}$ & native & local \\
\hline
\end{tabular}

